\title{
HOMOTOPY THEORY IN TORIC TOPOLOGY
}

\author{
J. GRBIĆ AND S. THERIAULT
}

\begin{abstract}
In toric topology, to each simplicial complex $K$ on $m$ vertices one associates two key spaces, the Davis-Januskiewicz space $D J_{K}$ and the moment-angle complex $\mathcal{Z}_{K}$, which are related by a homotopy fibration $\mathcal{Z}_{K} \stackrel{\widetilde{w}}{\longrightarrow} D J_{K} \longrightarrow \prod_{i=1}^{m} \mathbb{C} P^{\infty}$. A great deal of work has been done to study properties of $D J_{K}$ and $\mathcal{Z}_{K}$, their generalisations to polyhedral products, and applications to algebra, combinatorics and geometry.

In the first part of this paper we survey some of the main results in the study of the homotopy theory of these spaces. In the second part we break new ground by initiating a study of the map $\widetilde{w}$. We show that, for a certain family of simplicial complexes $K$, the map $\widetilde{w}$ is a sum of higher and iterated Whitehead products.
\end{abstract}

\section{Contents}

Part 1. A Survey of Homotopy Theory in Toric Topology 2

1. Introduction 2

1.1. Davis-Januszkiewicz spaces and moment-angle complexes 2

1.2. Connections to commutative algebra and combinatorics 3

1.3. Polyhedral products 4

1.4. Problems 6

2. Stable homotopy 6

3. Homology and Cohomology 9

3.1. Moment-angle complexes over simplicial posets 11

4. Rational homotopy theory 12

5. The homotopy type of $\mathcal{Z}_{K}$ and $(\underline{\mathrm{CX}}, \underline{\mathrm{X}})^{K}$ : Part I 14

6. The homotopy type of $\mathcal{Z}_{K}$ and $(\underline{\mathrm{CX}}, \underline{\mathrm{X}})^{K}$ : Part II 19

6.1. Vertex-decomposable, shellable and sequentially Cohen-Macaulay complexes 20

6.2. Configuration spaces and polyhedral products 22

6.3. Gluing along a common face 25

7. Minimally non-Golod complexes and connected sums of products of two spheres 28

Part 2. Higher Whitehead Products in Toric Topology 31

2010 Mathematics Subject Classification. Primary 55P35, 55Q15, Secondary 13F55, 52C35.

Key words and phrases. Davis-Januszkiewicz space, moment-angle complex, polyhedral product, homotopy type, higher Whitehead product, higher Samelson product. 
8. Statement of results 31

$\begin{array}{lll}9 . & \text { The objects of study } & 36\end{array}$

10. Higher Whitehead products and Fat wedges 39

11. Adams-Hilton models 41

12. An Adams-Hilton model for $F W(\underline{\mathrm{S}}) \quad 44$

13. Properties of $\Omega D J_{K}(\underline{\mathrm{S}})$ and $\Omega \mathcal{Z}_{K}(\underline{\mathrm{S}})$ for directed MF-complexes 48

14. Properties of $\Omega D J_{K}$ and $\Omega \mathcal{Z}_{K}$ for directed $M F$-complexes 54

15. Examples $\quad 60$

$\begin{array}{ll}\text { References } & 63\end{array}$

\section{Part 1. A Survey of Homotopy Theory in Toric Topology}

In the first part of the paper we survey some of the homotopy theory surrounding moment-angle complexes and their generalisation to polyhedral products. The topics covered are not meant to be comprehensive but instead reflect some of the problems and properties that have motivated research in the area over the past decade.

\section{INTRODUCTION}

1.1. Davis-Januszkiewicz spaces and moment-angle complexes. The story starts with Davis and Januszkiewicz's work [DJ] which uses simple polytopes to construct new families of manifolds with torus actions. However, it is more convenient for us to begin with Buchstaber and Panov's generalisation [BP1] of their construction to simplicial complexes. Let $K$ be a simplicial complex on the vertex set $[m]$. Let $T^{m}=\prod_{i=1}^{m} S^{1}$ be the $m$-torus and let $B T^{m}=\prod_{i=1}^{\infty} \mathbb{C} P^{\infty}$ be its classifying space. For a face $\sigma \in K$, let

$$
D J_{\sigma}=\prod_{i=1}^{m} Y_{i} \quad \text { where } \quad Y_{i}= \begin{cases}\mathbb{C} P^{\infty} & \text { if } i \in \sigma \\ * & \text { if } i \notin \sigma .\end{cases}
$$

The Davis-Januszkiewicz space $D J_{K}$ is defined by

$$
D J_{K}=\bigcup_{\sigma \in K} D J_{\sigma}
$$

Observe that $D J_{K}$ is a subspace of the product $\prod_{i=1}^{m} \mathbb{C} P^{\infty}$.

There is another fundamental space constructed analogously to $D J_{K}$. For a face $\sigma \in K$, let

$$
\mathcal{Z}_{\sigma}=\prod_{i=1}^{m} Y_{i} \quad \text { where } \quad Y_{i}= \begin{cases}D^{2} & \text { if } i \in \sigma \\ S^{1} & \text { if } i \notin \sigma .\end{cases}
$$

The moment-angle complex $\mathcal{Z}_{K}$ is defined by

$$
\mathcal{Z}_{K}=\bigcup_{\sigma \in K} \mathcal{Z}_{\sigma}
$$


Notice that $\mathcal{Z}_{K}$ is a subspace of the product $\prod_{i=1}^{m} D^{2}$. There is a canonical action of $T^{m}$ on $\prod_{i=1}^{m} D^{2}$ given by coordinate wise rotation of the disc. This induces an action of $T^{m}$ on the subspace $\mathcal{Z}_{K}$. A remarkable feature of this action is that the homotopy orbit space of $\mathcal{Z}_{K}$ is $D J_{K}$. Thus there is a homotopy fibration sequence

$$
T^{m} \longrightarrow \mathcal{Z}_{K} \longrightarrow D J_{K} \longrightarrow B T^{m}
$$

Davis and Januszkiewicz were interested in the case when the simplicial complex $K$ is the dual of the boundary of a simple polytope, in which case the moment-angle complex can be shown to be a manifold. They then considered characteristic maps $\lambda: T^{m} \longrightarrow T^{n}$ for $n<m$ satisfying certain condition (*) whose kernel ker $\lambda \simeq T^{m-n}$ had the property that the quotient space $M_{\lambda}=\mathcal{Z}_{K} / \operatorname{ker} \lambda$ was a manifold, equipped with an action of $T^{n}$. The manifolds $M_{\lambda}$ have come to be known as quasitoric manifolds and their geometry and algebraic topology are the subject of intense investigation. A good survey describing research in this direction is [CMS].

1.2. Connections to commutative algebra and combinatorics. The spaces $D J_{K}$ and $\mathcal{Z}_{K}$ are intimately related to concepts in commutative algebra and combinatorics. To describe connections to commutative algebra, let $R$ be a commutative ring with a unit and let $R\left[v_{1}, \ldots, v_{m}\right]$ be the graded polynomial algebra on $m$ variables, where $\operatorname{deg}\left(v_{i}\right)=2$ for each $i$. If $K$ is a simplicial complex on the vertex set $[m]$, the Stanley-Reisner ring $R[K]$, also known as the face ring of $K$, is the quotient ring

$$
R[K]=R\left[v_{1}, \ldots, v_{n}\right] / \mathcal{I}_{K}
$$

where $\mathcal{I}_{K}$ is the homogeneous ideal generated by all square free monomials $v^{\sigma}=v_{i_{1}} \cdots v_{i_{s}}$ such that $\sigma=\left\{v_{i_{1}}, \ldots v_{i_{s}}\right\} \notin K$. In [DJ, BP1] it is shown that there is a ring isomorphism

$$
H^{*}\left(D J_{K}\right) \cong \mathbb{Z}[K]
$$

Thus the algebraic topology of $D J_{K}$ can be used to determine information about the Stanley-Reisner ring $\mathbb{Z}[K]$ and vice-versa. Further, by $[\mathrm{BBP}]$ there is a ring isomorphism

$$
H^{*}\left(\mathcal{Z}_{K} ; \mathbb{Z}\right) \cong \operatorname{Tor}_{\mathbb{Z}\left[v_{1}, \ldots, v_{m}\right]}(\mathbb{Z}[K], \mathbb{Z}) \text {. }
$$

For a given $K$, the Tor-algebra may be very complex, involving non-trivial cup products or nontrivial higher Massey products. An important special case is when the Tor-algebra is as simple as possible. A local ring $R$ is Golod over $\mathbf{k}$ if all Massey products in $\operatorname{Tor}_{\mathbf{k}\left[v_{1}, \ldots, v_{m}\right]}(R, \mathbf{k})$ vanish, where $\mathbf{k}$ is a field or $\mathbb{Z}$. Golod [Go] showed that if $R$ is Golod then its Poincaré series is a rational function. It is a major problem in commutative algebra to determine which local rings are Golod. In our case, we ask when the Stanley-Reisner face ring $\mathbb{Z}[K]$ is Golod - which is equivalent to $H^{*}\left(\mathcal{Z}_{K}\right)$ having all cup products and higher Massey products being trivial. Trevisan [Tr] showed that the case of the Stanley-Reisner ring is sufficient in determining the Golod property of a local ring as polarisation of a local ring keeps the Golod property. 
To describe the connection to combinatorics, again assume that $K$ is a simplicial complex on the vertex set $[m]$. Let $L_{\sigma}$ be the complex coordinate subspace of $\mathbb{C}^{m}$ given by

$$
L_{\sigma}=\left\{\left(z_{1}, \ldots, z_{m}\right) \in \mathbb{C}^{m} \mid z_{i_{1}}=\cdots=z_{i_{k}}=0\right\}
$$

where $\sigma=\left\{i_{1}, \ldots, i_{k}\right\}$ is a subset of $[m]$. Taking the union of all such $L_{\sigma}$ for $\sigma \notin K$, we obtain a complex coordinate subspace arrangement

$$
\mathcal{C A}(K)=\bigcup_{\sigma \notin K} L_{\sigma}
$$

Its complement is

$$
U(K)=\mathbb{C}^{m} \backslash \mathcal{C} \mathcal{A}(K)
$$

Notice that the torus $T^{m}$ acts coordinate wise on each $L_{\sigma}$ and these actions are compatible over the union, so it acts on $\mathcal{C} \mathcal{A}(K)$ and therefore on its complement $U(K)$. Buchstaber and Panov [BP1] showed that the moment-angle complex $\mathcal{Z}_{K}$ is a $T^{m}$-equivariant retract of $U(K)$. Consequently, there is a homotopy equivalence

$$
U(K) \simeq \mathcal{Z}_{K}
$$

implying that homotopy theoretic properties of $\mathcal{Z}_{K}$ determine those of the complement of the corresponding coordinate subspace arrangement.

This interplay between Davis-Januszkiewicz spaces, moment-angle complexes, Stanley-Reisner rings, Golod rings and complements of coordinate subspace arrangements has motivated much of the research into the homotopy theory of $D J_{K}$ and $\mathcal{Z}_{K}$.

1.3. Polyhedral products. The analogous constructions of $D J_{K}$ and $\mathcal{Z}_{K}$ in (1) and (2) suggest that a generalised functorial construction. This led Buchstaber and Panov [BP2] to define $K$-powers which later in various work [GT1, DS, BBCG1] developed more fully as polyhedral products. Let $K$ be a simplicial complex on $m$ vertices. For $1 \leq i \leq m$, let $\left(X_{i}, A_{i}\right)$ be a pair of pointed $C W$ complexes, where $A_{i}$ is a pointed subspace of $X_{i}$. Let $(\underline{X}, \underline{A})=\left\{\left(X_{i}, A_{i}\right)\right\}_{i=1}^{m}$ be the sequence of pointed $C W$-pairs. For each simplex $\sigma \in K$, let $(\underline{X}, \underline{A})^{\sigma}$ be the subspace of $\prod_{i=1}^{m} X_{i}$ defined by

$$
(\underline{X}, \underline{A})^{\sigma}=\prod_{i=1}^{m} Y_{i} \quad \text { where } \quad Y_{i}=\left\{\begin{array}{cc}
X_{i} & \text { if } i \in \sigma \\
A_{i} & \text { if } i \notin \sigma .
\end{array}\right.
$$

The polyhedral product of $(\underline{X}, \underline{A})$ and $K$ is

$$
(\underline{X}, \underline{A})^{K}=\bigcup_{\sigma \in K}(\underline{X}, \underline{A})^{\sigma} .
$$

Notice that $(\underline{X}, \underline{A})^{K}$ is a subspace of the product $\prod_{i=1}^{m} X_{i}$. Notice as well that the polyhedral product construction $(\underline{X}, \underline{A})^{K}$ is natural for maps of pairs $\left(X_{i}, A_{i}\right) \longrightarrow\left(X_{i}^{\prime}, A_{i}^{\prime}\right)$ and for maps of simplicial complexes $K \longrightarrow L$ where $K$ and $L$ may have different vertex sets. If each pair $\left(X_{i}, A_{i}\right)$ 
equals a fixed pair $(X, A)$, write $(X, A)^{K}$ for the polyhedral product of $(\underline{X}, \underline{A})$ and $K$. Observe from (1) and (2) that $D J_{K}=\left(\mathbb{C} P^{\infty}, *\right)^{K}$ and $\mathcal{Z}_{K}=\left(D^{2}, S^{1}\right)^{K}$.

Polyhedral products are homotopy theoretic generalisations of Davis-Januszkiewicz spaces and moment-angle complexes, so it is reasonable to expect that most of the homotopy theoretical results that can be determined about $D J_{K}$ and $\mathcal{Z}_{K}$ also hold for the corresponding polyhedral products $(X, *)^{K}$ and $(C X, X)^{K}$, where $C X$ is the reduced cone on $X$. Of course, none of the geometry of moment-angle complexes, such as the existence of a manifold structure, can be expected to hold in general for polyhedral products, unless the pairs considered are $\left(D^{n}, S^{n-1}\right)$ for $n \geq 1$.

To get a feeling for polyhedral products it is helpful to describe several examples. For pointed spaces $X$ and $Y$, the smash product $X \wedge Y$ is the quotient space $X \wedge Y=(X \times Y) /(X \vee Y)$. Let $I$ be the unit interval, with 0 as basepoint. The (reduced) cone on $X$ is the quotient space $C X=(I \times X) / \sim$, where $(1, x) \sim\left(1, x^{\prime}\right)$ and $(t, *) \sim(0, *)$. The (reduced) join $X * Y$ is the quotient space $X * Y=(X \times I \times Y) / \sim$ where $(x, 0, y) \sim\left(x^{\prime}, 0, y\right),(x, 1, y) \sim\left(x, 1, y^{\prime}\right)$ and $(*, t, *) \sim(*, 0, *)$. It is well known that there is a natural homotopy equivalence $X * Y \simeq \Sigma X \wedge Y$. It is also well known that $X * Y$ is homotopy equivalent to the pushout

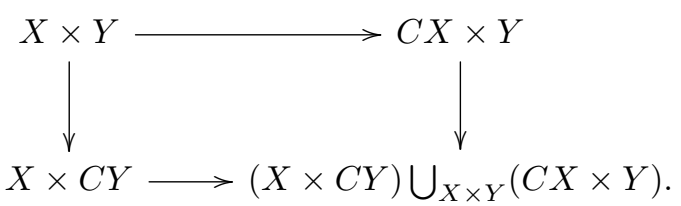

Examples:

(1) Suppose that each $A_{i}$ is a point. If $K$ is a disjoint union of $m$ points, then

$$
(\underline{X}, \underline{*})^{K}=X_{1} \vee \cdots \vee X_{m}
$$

and if $K$ is an $(m-1)$-simplex $\Delta^{m-1}$, then

$$
(\underline{X}, \underline{*})^{\Delta^{m-1}}=X_{1} \times \cdots \times X_{m} .
$$

(2) Again, suppose that each $A_{i}$ is a point. For $0 \leq i \leq m-1$, let $\Delta_{i}^{m-1}$ be the full $i$-skeleton of $\Delta^{m-1}$. Then

$$
(\underline{X}, \underline{*})^{\Delta_{i}^{m-1}}=\left\{\left(x_{1}, \ldots, x_{m}\right) \in \prod_{i=1}^{m} X_{i} \mid \text { at least } m-i-1 \text { of the } x_{i} \text { 's equals } *\right\} .
$$

The space on the right side is the $i^{t h}$-stage of Whitehead's filtration on $\prod_{i=1}^{m} X_{i}$.

(3) If $K$ is 2 disjoint points, then $\left(D^{2}, S^{1}\right)^{K}=D^{2} \times S^{1} \cup S^{1} \times D^{2}$, where the union is taken over $S^{1} \times S^{1}$. Therefore, $\left(D^{2}, S^{1}\right)^{K}$ is the boundary of $D^{4}$, that is, $S^{3}$. Since $D^{2}$ is contractible, $\left(D^{2}, S^{1}\right)^{K}$ is homotopy equivalent to the pushout of the maps $S^{1} \times S^{1} \longrightarrow C S^{1} \times S^{1}$ and $S^{1} \times S^{1} \longrightarrow S^{1} \times C S^{1}$. Thus

$$
\mathcal{Z}_{K}=\left(D^{2}, S^{1}\right)^{K} \simeq S^{1} * S^{1} \simeq S^{3} .
$$


(4) Generalising (3), if $K$ is 2 disjoint points then

$$
(\underline{C X}, \underline{X})^{K} \simeq X_{1} * X_{2}
$$

In particular,

$$
\left(D^{n+1}, S^{n}\right)^{K} \cong \partial D^{2 n+2} \simeq S^{n} * S^{n} \simeq S^{2 n+1} .
$$

Polyhedral products have found many uses beyond those involving Stanley-Reisner rings and complements of coordinate subspace arrangements. There is a large literature relating them to quasi-toric manifolds and small covers. Other applications include studying group actions on graphs, monodromy representations, configuration spaces of planar linkages, the $\mathbb{A}^{1}$-homotopy theory of smooth toric varieties, and assorted classes of arrangements. Their rising popularity is owned to the fact that they are complicated but approachable constructions which may be used to verify or predict conjectures.

1.4. Problems. We now have a large collection of spaces whose homotopy theory is interesting and connected to several other areas of mathematics. This gives rise to many natural problems, including:

(1) calculate the homology and cohomology of these spaces;

(2) determine their rational homotopy theory;

(3) determine their stable homotopy theory;

(4) determine families of simplicial complexes $K$ and $C W$-pairs $(X, A)$ for which the homotopy type of $(X, A)^{K}$ can be described precisely.

Many of these problems overlap and many lead to other questions, some of which will be indicated as we go.

Most of what follows will be developed in its general polyhedral product form as a great deal of the homotopy theory of the fundamental spaces, $D J_{K}$ and $\mathcal{Z}_{K}$, does not depend on properties specific to these spaces. But it is important to always keep in mind that it is the connections between algebraic topology, commutative algebra and combinatorics encoded by $D J_{K}$ and $\mathcal{Z}_{K}$ that drives most investigations.

\section{Stable homotopy}

We first consider the stable decomposition of $(\underline{X}, \underline{A})^{K}$ because it plays a central role in proving many other properties of polyhedral products. A stable decomposition implies a description of the homology of $(\underline{X}, \underline{A})^{K}$ and, remarkably, it can be used to determine the cup product structure in cohomology. It is also helpful when determining the homotopy type of particular families of polyhedral products because it acts as a bookkeeper, keeping track of vital information such as Poincaré series. 
The word stable may be a bit misleading, as the stable decomposition of $(\underline{X}, \underline{A})^{K}$ to be described occurs after only one suspension. However, it is a functorial decomposition which, stably, has no functorial refinement. It may be the case that a particular polyhedral product splits further after more suspensions but this arises from stable properties of the ingredient spaces rather than from the polyhedral product itself.

To begin we require the notion of a smash polyhedral product. Let $K$ be a simplicial complex on the vertex set $[m]$ and for $1 \leq i \leq m$ let $\left(X_{i}, A_{i}\right)$ be pairs of pointed $C W$-complexes. Recall that

$$
(\underline{X}, \underline{A})^{\sigma}=\prod_{i=1}^{m} Y_{i} \quad \text { where } \quad Y_{i}=\left\{\begin{array}{cc}
X_{i} & \text { if } i \in \sigma \\
A_{i} & \text { if } i \notin \sigma
\end{array}\right.
$$

and the polyhedral product is

$$
(\underline{X}, \underline{A})^{K}=\bigcup_{\sigma \in K}(\underline{X}, \underline{A})^{\sigma}
$$

For $\sigma \in K$, let

$$
\widehat{(\underline{X}, \underline{A})}^{\sigma}=\bigwedge_{i=1}^{m} Y_{i} \quad \text { where } \quad Y_{i}=\left\{\begin{array}{cl}
X_{i} & \text { if } i \in \sigma \\
A_{i} & \text { if } i \notin \sigma .
\end{array}\right.
$$

The smash polyhedral product is

$$
\widehat{(\underline{X}, \underline{A}}^{K}=\bigcup_{\sigma \in K} \widehat{(\underline{X}, \underline{A}}^{\sigma} .
$$

Another way to think of this is to recall that $(\underline{X}, \underline{A})^{K}$ is a subspace of the product $\prod_{i=1}^{m} X_{i}$, and $\widehat{(\underline{X}, \underline{A}}^{K}$ is the image of $(\underline{X}, \underline{A})^{K}$ under the natural quotient map $\prod_{i=1}^{m} X_{i} \longrightarrow \bigwedge_{i=1}^{m} X_{i}$.

As motivation for the stable decomposition, recall that there is a natural homotopy equivalence

$$
\Sigma\left(X_{1} \times X_{2}\right) \simeq \Sigma X_{1} \vee \Sigma X_{2} \vee\left(\Sigma X_{1} \wedge X_{2}\right)
$$

Iterating this decomposition gives a natural homotopy equivalence

$$
\Sigma\left(X_{1} \times \cdots \times X_{m}\right) \simeq \bigvee_{1 \leq i_{1}<\cdots<i_{k} \leq m} \Sigma X_{i_{1}} \wedge \cdots \wedge X_{i_{k}}
$$

The index set runs over all ordered subsequences of $[m]$ and therefore there is one-to-one correspondence with the faces in $\Delta^{m-1}$. Further, the polyhedral product $(\underline{X}, \underline{A})^{\Delta^{m-1}}$ is precisely the product $X_{1} \times \cdots \times X_{m}$, and each term $X_{i_{1}} \wedge \cdots \wedge X_{i_{k}}$ equals the smash polyhedral product $(\underline{X}, \underline{A}){ }^{K_{I}}$ for $I=\left\{i_{1}, \ldots, i_{k}\right\}$. So the previous homotopy equivalence can be equivalently rewritten as

$$
\Sigma(\underline{X}, \underline{A})^{\Delta^{m-1}} \simeq \bigvee_{I \subseteq[m]} \Sigma(\underline{X}, \underline{A})^{K_{I}} .
$$

This suggests that the suspension of a polyhedral product ought to be a wedge sum of suspensions of smash polyhedral products where the sum is taken over all full subcomplexes of $K$. This is exactly what Bahri, Bendersky, Cohen and Gitler proved in [BBCG1].

Let $K$ be a simplicial complex on the vertex set $[m]$ and for $1 \leq i \leq m$ let $\left(X_{i}, A_{i}\right)$ be pairs of pointed $C W$-complexes. Let $I \subset[m]$. Including $I$ into $[m]$, there is an induced inclusion $K_{I} \longrightarrow K$, 
which in turn induces a map of polyhedral products $(\underline{X}, \underline{A})^{K} \longrightarrow(\underline{X}, \underline{A})^{K_{I}}$. We then obtain a composition into a smash polyhedral product

$$
(\underline{X}, \underline{A})^{K} \longrightarrow(\underline{X}, \underline{A})^{K_{I}} \longrightarrow \widehat{(\underline{X}, \underline{A}}^{K_{I}}
$$

Suspending, we can add every such composition over all full subcomplexes of $K$, giving a composition

$$
\bar{H}: \Sigma(\underline{X}, \underline{A})^{K} \longrightarrow \bigvee_{I \subseteq[m]} \Sigma(\underline{X}, \underline{A})^{K_{I}} \longrightarrow \bigvee_{I \subseteq[m]} \Sigma(\underline{\underline{X}, \underline{A}})^{K_{I}} .
$$

Bahri, Bendersky, Cohen and Gitler [BBCG1, Theorem 2.10] proved the following.

Theorem 2.1. Let $K$ be a simplicial complex on the vertex set $[m]$ and for $1 \leq i \leq m$, let $\left(X_{i}, A_{i}\right)$ be pairs of pointed $C W$-complexes. The map

$$
\bar{H}: \Sigma(\underline{X}, \underline{A})^{K} \longrightarrow \bigvee_{I \subseteq[m]} \Sigma \widehat{(\underline{X}, \underline{A})}^{K_{I}}
$$

is a homotopy equivalence.

Further, in the special case when each $X_{i}$ is contractible, Bahri, Bendersky, Cohen and Gitler show

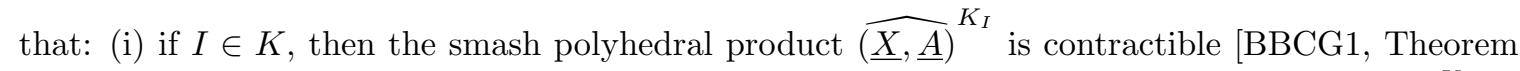
2.21]; and (ii) if $I \notin K$ and $I=\left(i_{1}, \ldots, i_{k}\right)$, then there is a homotopy equivalence $\widehat{(\underline{X}, \underline{A}}^{K_{I}} \simeq$ $\Sigma\left(\left|K_{I}\right| \wedge \widehat{A}^{I}\right)$ where $\widehat{A}^{I}=\bigwedge_{j=1}^{k} A_{i_{j}}$ [BBCG1, Theorem 2.19]. Specialising to the pairs $\left(C X_{i}, X_{i}\right)$, where $C X_{i}$ is the reduced cone on $X_{i}$, we have $(\underline{\widehat{C X}, \underline{X}})^{K_{I}} \simeq \Sigma\left(\left|K_{I}\right| \wedge \widehat{X}^{I}\right)$ and thus obtain the following.

Theorem 2.2. Let $K$ be a simplicial complex on the vertex set $[m]$ and for $1 \leq i \leq m$, let $X_{i}$ be a pointed $C W$-complex. Then the map $\bar{H}$ specialises to a homotopy equivalence

$$
H: \Sigma(\underline{C X}, \underline{X})^{K} \longrightarrow \bigvee_{I \notin K} \Sigma(\widehat{\widehat{C X}, \underline{X}})^{K_{I}} \stackrel{\simeq}{\longrightarrow} \bigvee_{I \notin K} \Sigma^{2}\left(\left|K_{I}\right| \wedge \widehat{X}^{I}\right)
$$

The key case is the moment-angle complex $\mathcal{Z}_{K}$. Here, each pair is $\left(D^{2}, S^{1}\right)$ and $D^{2}$ is contractible. Also, each $X_{i}=S^{1}$, so $\widehat{X}^{I}=\bigwedge_{j=1}^{k}\left(S^{1}\right)_{i_{j}} \simeq S^{k}$. Write $|I|=k$. We obtain the following corollary.

Corollary 2.3. Let $K$ be a simplicial complex on the vertex set $[m]$. There is a homotopy equivalence

$$
\Sigma \mathcal{Z}_{K} \longrightarrow \bigvee_{I \notin K} \Sigma^{|I|+2}\left|K_{I}\right|
$$




\section{Homology and Cohomology}

The cohomology of the Davis-Januszkiewicz space has already been discussed. There is a ring isomorphism

$$
H^{*}\left(D J_{K} ; \mathbb{Z}\right) \cong \mathbb{Z}[K]=\mathbb{Z}\left[v_{1}, \ldots, v_{m}\right] / \mathcal{I}_{K}
$$

where the left side is the Stanley-Reisner algebra (face ring of $K$ ) and $\mathcal{I}_{K}$ is the homogeneous ideal generated by all square free monomials $v^{\sigma}=v_{i_{1}} \cdots v_{i_{s}}$ such that $\sigma=\left\{v_{i_{1}}, \ldots v_{i_{s}}\right\} \notin K$.

The integral cohomology of the moment-angle complex $\mathcal{Z}_{K}$ was described as the Tor-algebra $\operatorname{Tor}_{\mathbb{Z}\left[v_{1}, \ldots, v_{m}\right]}(\mathbb{Z}[K], \mathbb{Z})$, but this may be difficult to compute explicitly. More tractably, there is a combinatorial description of the cup product structure in $\mathcal{Z}_{K}$ for any simplicial complex $K$, proved in $[\mathrm{BBP}, \mathrm{BP} 1, \mathrm{~F}]$. Take homology with integer coefficients. The join of two simplicial complexes $K_{1}$ and $K_{2}$ is $K_{1} * K_{2}=\left\{\sigma_{1} \cup \sigma_{2} \mid \sigma_{i} \in K_{i}\right\}$.

Theorem 3.1. There is an isomorphism of graded commutative algebras

$$
H^{*}\left(\mathcal{Z}_{K}\right) \cong \bigoplus_{I \subset[m]} \widetilde{H}^{*}\left(K_{I}\right)
$$

Here, $\widetilde{H}^{*}\left(K_{I}\right)$ denotes the reduced simplicial cohomology of the full subcomplex $K_{I} \subset K$. The isomorphism is the sum of isomorphisms

$$
H^{p}\left(\mathcal{Z}_{K}\right) \cong \sum_{I \subset[m]} \widetilde{H}^{p-|I|-1}\left(K_{I}\right)
$$

and the ring structure (the Hochster ring) is given by the maps

$$
H^{p-|I|-1}\left(K_{I}\right) \otimes H^{q-|J|-1}\left(K_{J}\right) \longrightarrow H^{p+q-|I|-|J|-1}\left(K_{I \cup J}\right)
$$

which are induced by the canonical simplicial maps $K_{I \cup J} \longrightarrow K_{I} * K_{J}$ for $I \cap J=\emptyset$ and zero otherwise.

Two key points in Theorem 3.1 are that, additively, the cohomology of $\mathcal{Z}_{K}$ is determined by that of its full subcomplexes, and multiplicatively, non-trivial cup products exist only when cohomology classes arise from disjoint full subcomplexes.

Bahri, Bendersky, Cohen and Gitler [BBCG2] generalised this to polyhedral products. The cup product for any space $M$ is induced by the diagonal map $\Delta: M \longrightarrow M \times M$. Composing $\Delta$ with the quotient map to the smash product, we obtain the reduced diagonal

$$
\widehat{\Delta}: M \longrightarrow M \wedge M
$$

Now suppose that $I \subseteq[m]$ and consider the space $Y^{I}=\prod_{i=1}^{m} Y_{i}$ where $Y_{i}=X_{i}$ if $i \in I$ and $Y_{i}=A_{i}$ if $i \notin I$. Then there is a reduced diagonal

$$
\widehat{\Delta}_{I}: \widehat{Y}^{I} \longrightarrow \widehat{Y}^{I} \wedge \widehat{Y}^{I} .
$$


Let $\pi_{I}: Y^{[m]} \longrightarrow Y^{I}$ be the projection and let $\widehat{\pi}_{I}$ be the composite

$$
\widehat{\pi}_{I}: Y^{[m]} \stackrel{\pi_{I}}{\longrightarrow} Y^{I} \longrightarrow \widehat{Y}^{I}
$$

Observe that if $I=J \cup L$, then the composite $Y^{[m]} \stackrel{\widehat{\Delta}}{\longrightarrow} Y^{[m]} \wedge Y^{[m]} \stackrel{\widehat{\pi}_{J} \wedge \widehat{\pi}_{L}}{\longrightarrow} \widehat{Y}^{J} \wedge \widehat{Y}^{L}$ factors through $\widehat{\pi}_{I}$. Thus there is a commutative diagram

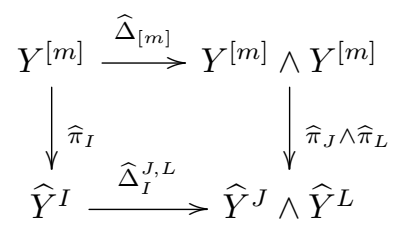

where $\widehat{\Delta}_{I}^{J, L}$ is the quotient map. Notice that if $I=J=L$ then $\widehat{\Delta}_{I}^{J, L}$ is the usual reduced diagonal. Restricting to $(\underline{X}, \underline{A})^{K} \subseteq X^{[m]}$, we obtain a commutative diagram

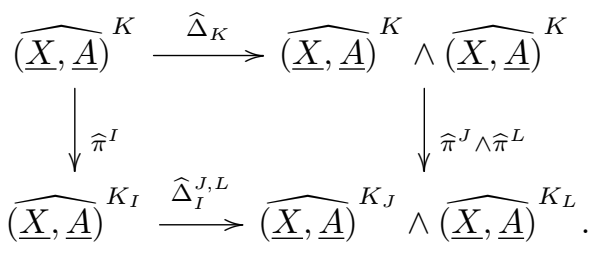

Let $u \in H^{p}\left((\underline{X}, \underline{A})^{K_{J}}\right)$ and $v \in H^{q}\left((\underline{X}, \underline{A})^{K_{L}}\right)$ be cohomology classes. The star product of $u$ and $v$ is

$$
u * v=\left(\widehat{\Delta}_{I}^{J, L}\right)^{*}(u \otimes v) \in H^{p+q}\left(\widehat{(\underline{X}, \underline{A}}^{K_{I}}\right) .
$$

The commutativity of (8) implies that

$$
\widehat{\pi}_{I}^{*}(u * v)={\widehat{\pi_{J}}}^{*}(u) \cup{\widehat{\pi_{L}}}^{*}(v)
$$

where $\cup$ is the cup product in $(\underline{X}, \underline{A})^{K}$. Bahri, Bendersky, Cohen and Gitler [BBCG2, Theorem 1.4] showed the following.

Theorem 3.2. Let $K$ be a simplicial complex on the vertex set $[m]$ and suppose that $(\underline{X}, \underline{A})=$ $\left\{\left(X_{i}, A_{i}\right)\right\}_{i=1}^{m}$ are pairs of pointed $C W$-complexes. Then there is a ring isomorphism

$$
H^{*}\left((\underline{X}, \underline{A})^{K}\right) \cong \oplus_{I \subseteq[m]} \widetilde{H}^{*}\left(\widehat{(\underline{X}, \underline{A}}^{K_{I}}\right)
$$

where the product structure on the right is given by the star product.

Theorem 3.2 corresponds to the first statement in Theorem 3.1 - the cohomology of $(\underline{X}, \underline{A})^{K}$ is determined by that of its full subcomplexes. The second statement connecting disjoint full subcomplexes to non-trivial cup products generalises to the case when each pair is a suspension by a simple observation: if $M$ is a suspension (or more generally, a co- $H$-space), then the reduced diagonal $M \stackrel{\widehat{M}}{\longrightarrow} M \wedge M$ is null homotopic. Suppose that each of the constituent pairs in $(\underline{X}, \underline{A})$ is a 
suspension, $\left(X_{i}, A_{i}\right)=\left(\Sigma X_{i}^{\prime}, \Sigma A_{i}^{\prime}\right)$. Suppose that $I=J \cup L$ and there is at least one $i \in J \cap L$. Consider the composite

$$
\theta: Y^{[m]} \stackrel{\widehat{\Delta}_{[m]}}{\longrightarrow} Y^{[m]} \wedge Y^{[m]} \stackrel{\widehat{\pi}_{J} \wedge \widehat{\pi}_{L}}{\longrightarrow} \widehat{Y}^{J} \wedge \widehat{Y}^{L}
$$

Since $i \in J \cap L$ there is a copy of $Y_{i}$ in both $\widehat{Y}^{J}$ and $\widehat{Y}^{L}$. Permuting the terms of $Y^{[m]}$ so $Y_{i}$ appears first, $\theta$ is the same as the composite

$$
Y_{i} \times Y^{[m] \backslash\{i\}} \stackrel{\widehat{\Delta}_{[m]}}{\longrightarrow}\left(Y_{i} \times Y^{[m] \backslash\{i\}}\right) \wedge\left(Y_{i} \times Y^{[m] \backslash\{i\}}\right) \stackrel{\widehat{\pi}_{J} \wedge \widehat{\pi}_{L}}{\longrightarrow}\left(Y_{i} \wedge \widehat{Y}^{J \backslash\{i\}}\right) \wedge\left(Y_{i} \wedge \widehat{Y}^{L \backslash\{i\}}\right) .
$$

By (7), this composite factors through

$$
\left(Y_{i} \wedge \widehat{Y}^{I \backslash\{i\}}\right) \stackrel{\widehat{\Delta}_{I}^{J, L}}{\longrightarrow}\left(Y_{i} \wedge \widehat{Y}^{J \backslash\{i\}}\right) \wedge\left(Y_{i} \wedge \widehat{Y}^{L \backslash\{i\}}\right) .
$$

But $\widehat{\Delta}_{I}^{J, L}$ factors through $Y_{i} \wedge \widehat{Y}^{I \backslash\{i\}} \stackrel{\widehat{\Delta} \wedge 1}{\longrightarrow} Y_{i} \wedge Y_{i} \wedge \widehat{Y}^{I \backslash\{i\}}$, which is null homotopic since $Y_{i}$ is a suspension. Therefore $\widehat{\Delta}_{I}^{J, K}$ is null homotopic. Restricting to $\widehat{(\underline{X}, \underline{A}}^{K} \subseteq X^{[m]}$, we obtain a null homotopy for $\widehat{(\underline{X}, \underline{A})}^{K_{I}} \stackrel{\widehat{\Delta}_{I}^{J, L}}{\longrightarrow} \widehat{(\underline{X}, \underline{A})}^{K_{J}} \wedge \widehat{(\underline{X}, \underline{A}}^{K_{L}}$. The latter map induces the star product in cohomology, so we obtain the following, proved in [BBCG2, Theorem 1.6].

Theorem 3.3. Let $K$ be a simplicial complex on the vertex set $[m]$ and suppose that $(\underline{X}, \underline{A})=$ $\left\{\left(X_{i}, A_{i}\right)\right\}_{i=1}^{m}$ are pairs of suspended pointed $C W$-complexes. Suppose that $I=J \cup L$ and $J \cap L \neq \emptyset$.

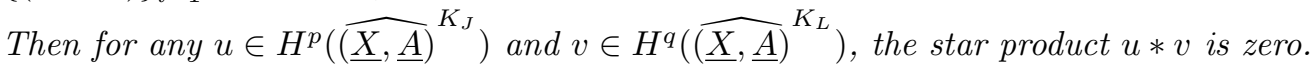

3.1. Moment-angle complexes over simplicial posets. As we saw before, one way of generalising a moment-angle complex $\mathcal{Z}_{K}=\left(D^{2}, S^{1}\right)^{K}$ is to replace the topological pair $\left(D^{2}, S^{1}\right)$ by any other topological pair or a sequence of pairs $(\underline{X}, \underline{A})$ to obtain a polyhedral product $(\underline{X}, \underline{A})^{K}$ determined by $(\underline{X}, \underline{A})$ and $K$. Another way of generalising $\mathcal{Z}_{K}$ is to replace a simplicial complex $K$ by a more general combinatorial object. This approach was taken by Lü, Maeda, Masuda and Panov [MMP, LP] who defined and studied moment-angle complexes over simplicial posets.

A poset (partially ordered set) $\mathcal{S}$ with the order relation $\leq$ is called simplicial if it has an initial element $\hat{0}$ and for each $\sigma \in \mathcal{S}$ the lower segment $[\hat{0}, \sigma]$ is a boolean lattice (the face poset of a simplex). We assume all posets to be finite and refer to elements $\sigma \in \mathcal{S}$ as simplices. The rank function $|\cdot|$ on $\mathcal{S}$ is defined by setting $|\sigma|=k$ for $\sigma \in \mathcal{S}$ if $[\hat{0}, \sigma]$ is the face poset of a $(k-1)$-dimensional simplex. The vertices of $\mathcal{S}$ are elements of rank one. We assume that $\mathcal{S}$ has $m$ vertices and denote the vertex set by $V(\mathcal{S})=[m]$. Similarly we denote by $V(\sigma)$ the vertex set of $\sigma$, that is the set of $i$ with $i \leq \sigma$.

To any simplicial poset $\mathcal{S}$, an algebraic object, the face ring of $\mathcal{S}$ can be associated.

Definition 3.4. $[\mathrm{S}]$ The face ring of a simplicial poset $\mathcal{S}$ is the quotient

$$
\mathbb{Z}[\mathcal{S}]=\mathbb{Z}\left[v_{\sigma} \mid \sigma \in \mathcal{S}\right] / \mathcal{I}_{\mathcal{S}}
$$

where $\mathcal{I}_{\mathcal{S}}$ is the ideal generated by the elements $v_{\hat{0}}-1$ and

$$
v_{\sigma} v_{\tau}-v_{\sigma \wedge \tau} \cdot \sum_{\eta \in \sigma \vee \tau} v_{\eta}
$$


The ring $\mathbb{Z}[\mathcal{S}]$ generalises the Stanley-Reisner ring $\mathbb{Z}[K]$ of a simplicial complex $K$. The ring $\mathbb{Z}[\mathcal{S}]$ have remarkable algebraic and homological properties, albeit they are much more complicated than the Stanley-Reisner rings $\mathbb{Z}[K]$. Unlike $\mathbb{Z}[K]$, the ring $\mathbb{Z}[\mathcal{S}]$ is not generated in the lowest positive degree which makes their study more difficult.

To define a moment-angle complex over a simplicial poset, we need to set some notation. Using a formal categorical language, we consider the face category $\operatorname{CAT}(\mathcal{S})$ whose objects are elements $\sigma \in \mathcal{S}$ and there is a morphism from $\sigma$ to $\tau$ whenever $\sigma \leq \tau$.

For every $\sigma \in \mathcal{S}$, consider the following subset in $\left(D^{2}\right)^{m}$

$$
B_{\sigma}=\left\{\left(z_{1}, \ldots, z_{m} \in\left(D^{2}\right)^{m}|| z_{j} \mid=1 \text { if } j \not \leq \sigma\right\} .\right.
$$

Then $B_{\sigma}$ is homeomorphic to a product of $|\sigma|$ discs and $m-|\sigma|$ circles. We have an inclusion $B_{\tau} \subset B_{\sigma}$ whenever $\tau \leq \sigma$. It follows that the assignment $\sigma \mapsto B_{\sigma}$ defines a diagram from $\operatorname{CAT}(\mathcal{S})$ to TOP, which we denote by $\left(D^{2}, S^{1}\right)^{\mathcal{S}}$.

Definition 3.5. The moment-angle complex corresponding to a simplicial poset $\mathcal{S}$ is

$$
\mathcal{Z}_{S}=\operatorname{colim}\left(D^{2}, S^{1}\right)^{\mathcal{S}}
$$

from $\operatorname{CAT}(\mathcal{S})$ to TOP.

The space $\mathcal{Z}_{\mathcal{S}}$ has many important topological properties of the original moment-angle complex $\mathcal{Z}_{K}$ associated to a simplicial complex $K$. Lü and Panov [LP] proved that the integral cohomology algebra of $\mathcal{Z}_{\mathcal{S}}$ is isomorphic to the Tor-algebra of the face ring $\mathbb{Z}[\mathcal{S}]$. This led directly to a generalisation of Hochster's theorem, expressing the algebraic Betti numbers of the ring $\mathbb{Z}[\mathcal{S}]$ in terms of the homology of full subposets of $\mathcal{S}$.

Theorem 3.6. There is a graded ring isomorphism

$$
H^{*}\left(\mathcal{Z}_{\mathcal{S}} ; \mathbb{Z}\right) \cong \operatorname{Tor}_{\mathbb{Z}\left[v_{1}, \ldots, v_{m}\right]}(\mathbb{Z}[\mathcal{S}], \mathbb{Z})
$$

whose graded componetns are given by the group isomorphisms

$$
H^{p}\left(\mathcal{Z}_{\mathcal{S}} ; \mathbb{Z}\right) \cong \oplus_{-i+2|a|=p} \operatorname{Tor}_{\mathbb{Z}\left[v_{1}, \ldots, v_{m}\right]}^{-i,|a|}(\mathbb{Z}[\mathcal{S}], \mathbb{Z})
$$

in each degree $p$. Here $|a|=j_{1}+\cdots+j_{m}$ for $a=\left(j_{1}, \ldots, j_{m}\right)$.

\section{Rational homotopy theORY}

Rational homotopy theory is one of the great success stories in algebraic topology. The rational homotopy type of (nilpotent, finite type) spaces is completely determined by algebraic invariants. The most common way of calculating the rational homotopy theory of a space or a map is to use a Sullivan minimal model, although there are other models and any would do. 
Formally, a rational model of a connected space $X$ is a commutative differential graded $\mathbb{Q}$-algebra $\mathcal{M}$ which is connected, of finite type and quasi-isomorphic to the commutative differential graded algebra $A P_{L}(X)$ of polynomial differential forms on $\mathrm{X}$ with rational coefficients. Félix and Tanré [FT] constructed a model for the rational homotopy theory of any polyhedral product and used it to prove their rational properties. To describe their results we need some notation.

Let $(\underline{X}, \underline{A})=\left\{\left(X_{i}, A_{i}\right)\right\}_{i=1}^{m}$ be pairs of pointed $C W$-complexes which are nilpotent and of finite type. For each pair $\left(X_{i}, A_{i}\right)$ choose a surjective model $\varphi_{i}: \mathcal{M}_{i} \longrightarrow \mathcal{M}_{i}^{\prime}$ of the inclusion $A_{i} \longrightarrow X_{i}$. For each $\sigma \subset[m]$, let $I_{\sigma}$ be the ideal of $\otimes_{i=1}^{m} \mathcal{M}_{i}$ defined by

$$
I_{\sigma}=E_{1} \otimes \cdots \otimes E_{n} \quad \text { where } \quad E_{i}= \begin{cases}\operatorname{ker}\left(\varphi_{i}\right) & \text { if } i \in \sigma \\ \mathcal{M}_{i} & \text { if } i \notin \sigma .\end{cases}
$$

Let

$$
I(K)=\Sigma_{\sigma \notin K} I_{\sigma} .
$$

Note the resemblance between $\otimes_{i=1}^{m} \mathcal{M}_{i} / I(K)$ and the cohomology of the Davis-Januskiewicz space, $H^{*}\left(D J_{K} ; \mathbb{Z}\right) \cong \mathbb{Z}\left[v_{1}, \ldots, v_{m}\right] / \mathcal{I}_{K}$ where $\mathcal{I}_{K}$ is the homogeneous ideal generated by all square free monomials $v^{\sigma}=v_{i_{1}} \cdots v_{i_{k}}$ for $\sigma=\left\{v_{i_{1}}, \ldots, v_{i_{k}}\right\} \notin K$. Félix and Tanré [FT] proved the following.

Theorem 4.1. Let $(\underline{X}, \underline{A})=\left\{\left(X_{i}, A_{i}\right)\right\}_{i=1}^{m}$ be pairs of pointed $C W$-complexes which are nilpotent and of finite type. Choose a surjective rational model $\varphi_{i}: \mathcal{M}_{i} \longrightarrow \mathcal{M}_{i}^{\prime}$ of the inclusion $A_{i} \longrightarrow X_{i}$. Then the following hold:

(a) the quotient $\otimes_{i=1}^{m} \mathcal{M}_{i} / I(K)$ is a rational model of $(\underline{X}, \underline{A})^{K}$;

(b) if $J \subset K$ is a subcomplex, then the projection

$$
\varphi_{K, J}: \otimes_{i=1}^{m} \mathcal{M}_{i} / I(K) \longrightarrow \otimes_{i=1}^{m} \mathcal{M}_{i} / I(J)
$$

is a rational model of the inclusion $(\underline{X}, \underline{A})^{J} \longrightarrow(\underline{X}, \underline{A})^{K}$.

As with any rational space, the most interesting point is not that there is a good model for it - Sullivan minimal models are guaranteed to exist, although tractable descriptions may be hard to obtain - but what can be done with it.

A rational space $X$ is formal if $H^{*}(X ; \mathbb{Q})$ is a model for $X$. Suppose that each $X_{i}=X$ and $A_{i}=*$. The model for the polyhedral product in Theorem 4.1 immediately shows that if $X$ is formal then so is $(X, *)^{K}$. This recovers the rational case of a more general result on formality that holds for cohomology coefficients in any commutative ring by Notbohm and Ray [NR].

A rational space $X$ is elliptic if $\pi_{*}(X)$ has finitely many $\mathbb{Q}$-summands. Otherwise it is hyperbolic. These notions have been well studied in rational homotopy theory and a remarkable fact is that if a rational space is hyperbolic then the number of rational homotopy groups grows exponentially with the degree $d$ of $\pi_{d}(X)$. That is, there are no non-elliptic rational spaces whose rational homotopy 
groups have polynomial growth. Félix and Tanré use their model for the polyhedral product to show that if each $X_{i}=X$ and $A_{i}=*$ then $(X, *)^{K}$ is hyperbolic provided $K \neq \Delta^{m-1}$ and $H^{*}(X ; \mathbb{Q})=\mathbb{Q}[v]$ for some $v$ of even degree. For the $C W$-pair $\left(D^{2}, S^{1}\right)$, Bahri, Bendersky, Cohen and Gitler [BBCG3] showed that the only moment-angle complexes $\mathcal{Z}_{K}$ that are rationally elliptic are those which are products of odd dimensional spheres and a disk, that is, $\mathcal{Z}_{K}$ for $K$ being a join of the boundary of simplices.

\section{The homotopy type of $\mathcal{Z}_{K}$ And $(\underline{C X}, \underline{X})^{K}$ : Part I}

Motivation for determining the homotopy type of the moment-angle complex $\mathcal{Z}_{K}$ comes from its equivalent description, up to equivariant homotopy, as the complement of a coordinate subspace arrangement, as discussed in Section 1. A major problem in combinatorics for many years was to describe families of simplicial complexes $K$ for which the complement of the corresponding coordinate subspace arrangement $U(K)$ was homotopy equivalent to a wedge of spheres. This problem was motivated by the fact that well-studied complements of hyperplane arrangements break into a wedge of spheres after being suspended twice. The complement $U(K)$ is substantially different as it may contain any cohomology torsion. Great progress has been achieved in resolving this problem using methods from homotopy theory to analyse the homotopy type of $\mathcal{Z}_{K}$. In fact, the methods used are general enough to allow for a simultaneous analysis of the homotopy types of the polyhedral product $(\underline{C X}, \underline{X})^{K}$.

To begin the discussion, we start with some definitions from combinatorics. Let $K$ be a simplicial complex on the vertex set $[m]$. For a simplex $\sigma \in K$, the star, restriction (or deletion) and link of $\sigma$ are the subcomplexes

$$
\begin{aligned}
\operatorname{star}_{K}(\sigma) & =\{\tau \in K \mid \sigma \cup \tau \in K\} ; \\
K \backslash \sigma=K_{[m] \backslash \sigma} & =\{\tau \in K \mid \sigma \cap \tau=\emptyset\} ; \\
\operatorname{link}_{K}(\sigma) & =\operatorname{star}_{K}(\sigma) \cap K \backslash \sigma .
\end{aligned}
$$

Recall that the join of two simplicial complexes $K_{1}, K_{2}$ on disjoint index sets is the simplicial complex

$$
K_{1} * K_{2}=\left\{\sigma_{1} \cup \sigma_{2} \mid \sigma_{i} \in K_{i}\right\}
$$

From the definitions, it follows that for a vertex $\{v\} \in K$, the $\operatorname{star} \operatorname{star}_{K}(v)$ is a join,

$$
\operatorname{star}_{K}(v)=\{v\} * \operatorname{link}_{K}(v),
$$

and there is a pushout

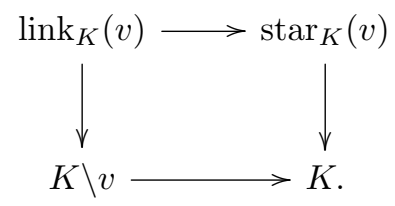


Consider the vertex $\{m\} \in K$. To compare the polyhedral products for $K, \operatorname{link}_{K}(m), \operatorname{star}_{K}(m)$ and $K \backslash\{m\}$, we should really consider each simplicial complex as being on the vertex set $[m]$. This changes nothing for $K$ and $\operatorname{star}_{K}(m)$ as their vertex sets are already $[m]$ but it introduces a "ghost" vertex $\{m\}$ for $\operatorname{link}_{K}(m)$ and $K \backslash\{m\}$. Let $\overline{\operatorname{link}_{K}(m)}$ and $\overline{K \backslash\{m\}}$ be the simplicial complexes $\operatorname{link}_{K}(m)$ and $K \backslash\{m\}$, regarded as simplicial complexes on the vertex set $[m]$. Then from pushout (9), we obtain a pushout of polyhedral products

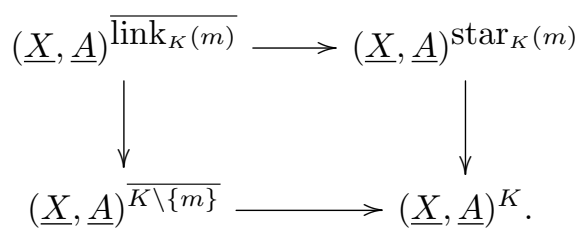

The spaces and maps in (10) can be better identified. First, the definition of the polyhedral product implies that

$$
\begin{gathered}
(\underline{X}, \underline{A})^{\overline{\operatorname{link}_{K}(m)}}=(\underline{X}, \underline{A})^{\operatorname{link}_{K}(m)} \times A_{m} \\
(\underline{X}, \underline{A})^{\overline{K \backslash\{m\}}}=(\underline{X}, \underline{A})^{K \backslash\{m\}} \times A_{m} .
\end{gathered}
$$

The inclusion $\operatorname{link}_{K}(m) \longrightarrow K \backslash\{m\}$ therefore implies that the induced map of polyhedral products $(\underline{X}, \underline{A})^{\overline{\operatorname{lnk}_{K}(m)}} \longrightarrow(\underline{X}, \underline{A})^{\overline{K \backslash\{m\}}}$ is the same as the product map $(\underline{X}, \underline{A})^{\operatorname{link}_{K}(m)} \times A_{m} \longrightarrow$ $(\underline{X}, \underline{A})^{K \backslash\{m\}} \times A_{m}$.

Second, the definition of the join of two simplicial complexes implies that if $K=K_{1} * K_{2}$ then there is an identity of topological spaces

$$
(\underline{X}, \underline{A})^{K} \cong(\underline{X}, \underline{A})^{K_{1}} \times(\underline{X}, \underline{A})^{K_{2}} .
$$

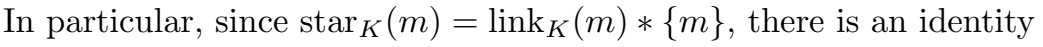

$$
(\underline{X}, \underline{A})^{\operatorname{star}(K)(m)} \cong(\underline{X}, \underline{A})^{\operatorname{link}_{K}(m)} \times X_{m} .
$$

We may also regard $\overline{\operatorname{lnk}_{K}(m)}$ as $\operatorname{link}_{K}(m) * \emptyset$, where $\emptyset$ corresponds to the ghost vertex at $\{m\}$. The inclusion $\operatorname{link}_{K}(m) \longrightarrow \operatorname{star}_{K}(m)$ therefore induces a map of joins

$$
\varlimsup \overline{\operatorname{link}}_{K}(m)=\operatorname{link}_{K}(m) * \emptyset \longrightarrow \operatorname{link}_{K}(m) *\{m\}=\operatorname{star}_{K}(m) .
$$

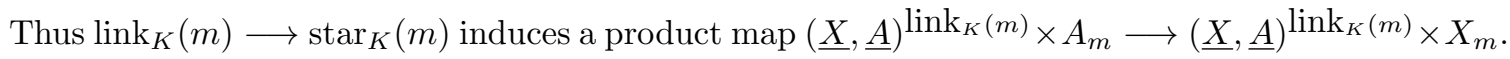

Combining these two observations, (10) can be rewritten as a pushout of polyhedral products

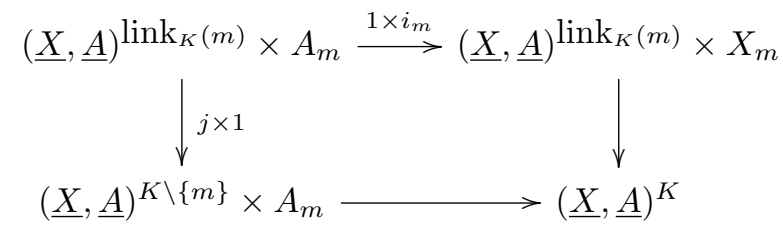

where $i_{m}$ is the inclusion and $j$ is the map of polyhedral products induced by the inclusion $\operatorname{link}_{K}(m) \longrightarrow$ $K \backslash\{m\}$. 
Now take the special case where each pair $\left(X_{i}, A_{i}\right)$ has $X_{i}$ being contractible. Recall that it is standard to adjust notation now, and consider pairs $(\underline{C X}, \underline{X})=\left\{\left(C X_{i}, X_{i}\right)\right\}_{i=1}^{m}$, where $C X_{i}$ is the reduced cone on $X_{i}$. Then the map $(\underline{C X}, \underline{X})^{\operatorname{link}_{K}(m)} \times X_{m} \stackrel{1 \times i_{m}}{\longrightarrow}(\underline{C X}, \underline{X})^{\operatorname{link}_{K}(m)} \times C X_{m}$ is equivalent, up to homotopy, to the map $(\underline{C X}, \underline{X})^{\operatorname{link}_{K}(m)} \times X_{m} \stackrel{\pi_{1}}{\longrightarrow}(\underline{C X}, \underline{X})^{\operatorname{link}_{K}(m)}$, where $\pi_{1}$ is the projection onto the first factor. Thus (11) specialises to a homotopy commutative diagram

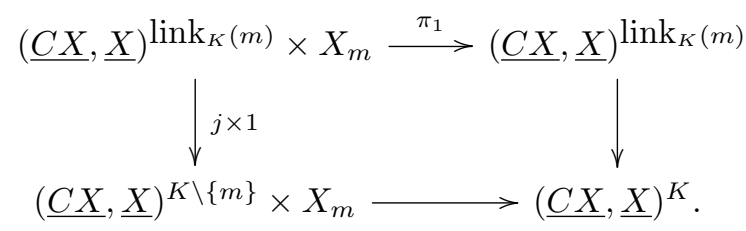

Pushout (12) is a key part of the work of Grbić-Theriault [GT2, GT3], Iriye-Kishimoto [IK1] and Grujić-Welker $[\mathrm{GW}]$ that determined families of simplicial complexes for which the homotopy type of $(\underline{C X}, \underline{X})^{K}$ can be identified as a wedge of iterated smashes of the $X_{i}$ 's. The important homotopy theoretic observation is the following, first proved in [GT1]. The (right) half-smash of two pointed spaces $C$ and $B$ is the quotient space $C \rtimes B=C \times B / \sim$ where $(*, b) \sim *$. It is well known that if $C$ is a co- $H$-space then $C \rtimes B \simeq C \vee(C \wedge B)$.

Lemma 5.1. Suppose that there is a homotopy pushout

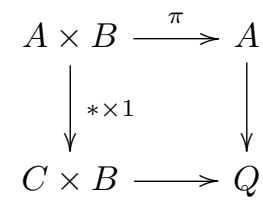

where $\pi$ is the projection. Then there is a homotopy equivalence $Q \simeq(A * B) \vee(C \rtimes B)$.

Now suppose that $K$ is a simplicial complex for which the map $(\underline{C X}, \underline{X})^{\operatorname{link}_{K}(m)} \longrightarrow(\underline{C X}, \underline{X})^{K \backslash\{m\}}$ is null homotopic. Then Lemma 5.1 can be applied to the homotopy pushout (12) to obtain a homotopy equivalence

$$
(\underline{C X}, \underline{X})^{K} \simeq\left((\underline{C X}, \underline{X})^{\operatorname{link}_{K}(m)} * X_{m}\right) \vee\left((\underline{C X}, \underline{X})^{K \backslash\{m\}} \rtimes X_{m}\right) .
$$

Definition 5.2. Let $\mathcal{W}_{m}$ be the collection of spaces which are homotopy equivalent to a wedge of suspensions of iterated smashes of the form $X_{i_{1}} \wedge \cdots \wedge X_{i_{k}}$ for $1 \leq i_{1}<\cdots<i_{k} \leq m$.

Suppose that $(\underline{C X}, \underline{X})^{\operatorname{link}_{K}(m)} \in \mathcal{W}_{m}$ and $(\underline{C X}, \underline{X})^{K \backslash\{m\}} \in \mathcal{W}_{m}$. Then as $(\underline{C X}, \underline{X})^{K \backslash\{m\}}$ is a suspension, we obtain

$$
(\underline{C X}, \underline{X})^{K \backslash\{m\}} \rtimes X_{m} \simeq(\underline{C X}, \underline{X})^{K \backslash\{m\}} \vee\left((\underline{C X}, \underline{X})^{K \backslash\{m\}} \wedge X_{m}\right)
$$

and a homotopy equivalence

$$
(\underline{C X}, \underline{X})^{K} \simeq\left((\underline{C X}, \underline{X})^{\operatorname{link}_{K}(m)} * X_{m}\right) \vee(\underline{C X}, \underline{X})^{K \backslash\{m\}} \vee\left((\underline{C X}, \underline{X})^{K \backslash\{m\}} \wedge X_{m}\right)
$$


implying that

$$
(\underline{C X}, \underline{X})^{K} \in \mathcal{W}_{m}
$$

Notice that in the special case of primary interest, the moment-angle complex $\mathcal{Z}_{K}$, each $X_{i}=S^{1}$ so a suspension of an iterated smash of $X_{i}$ 's is homotopy equivalent to a sphere. Therefore in this case $\mathcal{W}_{m}$ consists of those spaces which are homotopy equivalent to a wedge of spheres. So if $\mathcal{Z}_{\text {link }_{K}(m)} \in \mathcal{W}_{m}$, and $\mathcal{Z}_{K \backslash\{m\}} \in \mathcal{W}_{m}$ and the map $\mathcal{Z}_{K \backslash\{m\}} \stackrel{j}{\longrightarrow} \mathcal{Z}_{K \backslash\{m\}}$ is null homotopic then $\mathcal{Z}_{K} \in \mathcal{W}_{m}$, that is, $\mathcal{Z}_{K}$ is homotopy equivalent to a wedge of spheres.

The strategy is therefore to find a family $\mathcal{K}$ of simplicial complexes where, ideally: (i) if $K \in \mathcal{K}$ then $\operatorname{link}_{K}(m) \in \mathcal{K}$ and $K \backslash\{m\} \in \mathcal{K}$ so an inductive procedure can be established, and (ii) the induced map of polyhedral products $(\underline{C X}, \underline{X})^{\operatorname{link}_{K}(m)} \longrightarrow(\underline{C X}, \underline{X})^{K \backslash\{m\}}$ is null homotopic.

The first such family was shifted complexes. A simplicial complex $K$ is shifted if there is an ordering on its vertices such that whenever $\sigma \in K$ and $\nu^{\prime}<\nu$, then $(\sigma-\nu) \cup \nu^{\prime} \in K$. Examples include any full $i$-skeleton of the simplex $\Delta^{m-1}$. The definition of a shifted complex implies that if $K$ is shifted then both $\operatorname{link}_{K}(m)$ and $K \backslash\{m\}$ are shifted. In [GT3, IK1] the main work is in showing that the map $(\underline{C X}, \underline{X})^{\operatorname{link}_{K}(m)} \longrightarrow(\underline{C X}, \underline{X})^{K \backslash\{m\}}$ is null homotopic, implying the following.

Theorem 5.3. Let $K$ be a shifted complex. Then

$$
(\underline{C X}, \underline{X})^{K} \in \mathcal{W}_{m}
$$

In particular, the moment-angle complex $\mathcal{Z}_{K}$ is homotopy equivalent to a wedge of spheres.

The statement in Theorem 5.3 can be made more precise, in the sense that the wedge summands in the decomposition for $(\underline{C X}, \underline{X})^{K}$ can be made explicit. The best way to do this is to relate the decomposition to the stable decomposition for $\Sigma(\underline{C X}, \underline{X})^{K}$ in Theorem 2.1. The wedge summands for the stable decomposition are of the form $\left|K_{I}\right| * \widehat{X}^{I}$ for $I \notin K$. This implies that if $\mathcal{Z}_{K}$ were to be a wedge of spheres all full subcomplexes $\left|K_{I}\right|$ for $I \notin K$ after $I$-fold suspension must be homotopy equivalent to a wedge of spheres. When $K$ is shifted, each $\left|K_{I}\right|$ is homotopy equivalent to a wedge of spheres, so $\left|K_{I}\right| * \widehat{X}^{I} \in \mathcal{W}_{m}$. The explicit version of Theorem 5.3 proved in [GT3, IK1] says that the decomposition for $\Sigma(\underline{C X}, \underline{X})^{K}$ desuspends.

Theorem 5.4. Let $K$ be a shifted complex. Then there is a homotopy equivalence

$$
(\underline{C X}, \underline{X})^{K} \simeq \bigvee_{I \notin K}\left(\left|K_{I}\right| * \widehat{X}^{I}\right)
$$

In particular, if $|I|$ equals the cardinality of I then there is a homotopy equivalence

$$
\mathcal{Z}_{K} \simeq \bigvee_{I \notin K}\left(\left|K_{I}\right| \wedge S^{|I|+1}\right)
$$


In earlier work of the authors [GT2] which used different methods, Theorem 5.3 was proved in the special case when each pair $\left(C X_{i}, X_{i}\right)$ has $X_{i} \simeq \Omega X_{i}^{\prime}$ for some space $X_{i}^{\prime}$. Note that when each pair is $\left(D^{2}, S^{1}\right)$ then $S^{1} \simeq \Omega \mathbb{C} P^{\infty}$, so the homotopy equivalence for $\mathcal{Z}_{K}$ follows.

Theorem 5.4 generalizes results in classical homotopy theory proved by Ganea and Porter in the 1960s. For $0 \leq k \leq m$, let

$$
T_{m-k}^{m}=\left\{\left(x_{1}, \ldots, x_{m}\right) \in \prod_{i=1}^{m} X_{i} \mid \text { at least } k \text { coordinates } x_{i} \text { equal } * .\right\}
$$

Notice that there are subspace inclusions

$$
T_{0}^{m} \subseteq T_{1}^{m} \subseteq \cdots \subseteq T_{m}^{m} \subseteq \prod_{i=1}^{m} X_{i}
$$

This is Whitehead's filtration of the product $\prod_{i=1}^{m} X_{i}$. Several of these spaces are known by other names: observe that $T_{0}^{m} \simeq *, T_{1}^{m}=X_{1} \vee \cdots \vee X_{m}, T_{m-1}^{m}$ is the fat wedge and $T_{m}^{m}=\prod_{i=1}^{m} X_{i}$. Including $T_{m-k}^{m}$ into the product, define the space $F_{m-k}^{m}$ by the homotopy fibration

$$
F_{m-k}^{m} \longrightarrow T_{m-k}^{m} \longrightarrow \prod_{i=1}^{m} X_{i}
$$

A natural problem is to try to determine the homotopy type of $F_{m-k}$. Ganea [Ga] showed that $F_{1}^{2}$, the homotopy fibre of the inclusion $X_{1} \vee X_{2} \longrightarrow X_{1} \times X_{2}$, is homotopy equivalent to $\Omega X_{1} * \Omega X_{2}$ and Porter generalised this to $F_{m-k}^{m}$ in a way that will be described momentarily.

First, we rephrase the problem in terms of polyhedral products. Observe that $T_{m-k}^{m}=(\underline{X}, \underline{*})^{K}$ for $K=\Delta_{m-k}^{m-1}$, that is, $K$ is the full $(m-k)$-skeleton of the standard $(m-1)$-simplex. Observe as well that $\prod_{i=1}^{m} X_{i}=(\underline{X}, \underline{X})^{K}$ for the same $K$. Bahri, Bendersky, Cohen and Gilter [BBCG1], relying heavily on a theorem of Denham and Suciu [DS], proved a general result regarding homotopy fibrations in this kind of setting. For a space $X$, let $P X$ be the path space on $X$.

Theorem 5.5. For $1 \leq i \leq m$, let $X_{i}$ be a pointed $C W$-complex. Let $K$ be a simplicial complex on the vertex set $[m]$. There is a homotopy fibration

$$
(\underline{P X}, \underline{\Omega X})^{K} \longrightarrow(\underline{X}, \underline{*})^{K} \longrightarrow(\underline{X}, \underline{X})^{K}
$$

It is usual to replace $(\underline{P X}, \underline{\Omega X})$ with $(\underline{C \Omega X}, \underline{\Omega X})$ where $C \Omega X$ is the reduced cone on $\Omega X$. This is done via a natural homotopy equivalence of pairs $(P Y, \Omega Y) \simeq(C \Omega Y, \Omega Y)$. Doing so, and rewriting $(\underline{X}, \underline{X})^{K}$ as $\prod_{i=1}^{m} X_{i}$, we obtain a homotopy fibration

$$
(\underline{C \Omega X}, \underline{\Omega X})^{K} \longrightarrow(\underline{X}, \underline{*})^{K} \longrightarrow \prod_{i=1}^{m} X_{i}
$$

In particular, taking $K=\Delta_{m-k}^{m-1}$ we have a model for the homotopy fibration $F_{m-k}^{m} \longrightarrow T_{m-k}^{m} \longrightarrow$ $\prod_{i=1}^{m} X_{i}$. 
This lets us write Porter's result in terms of polyhedral products. Porter [Po2] showed that for any simply-connected spaces $X_{1}, \ldots, X_{m}$ there is a homotopy equivalence

$$
(\underline{C \Omega X}, \underline{\Omega X})^{K} \simeq \bigvee_{j=k+2}^{m}\left(\bigvee_{1 \leq i_{1}<\cdots<i_{j} \leq m}\left(\begin{array}{l}
j-1 \\
k+1
\end{array}\right) \Sigma^{k+1} \Omega X_{i_{1}} \wedge \cdots \wedge \Omega X_{i_{j}}\right) .
$$

Theorem 5.3 generalizes this. If $X_{1}, \ldots, X_{m}$ are any path-connected spaces then there is a homotopy equivalence

$$
(\underline{C X}, \underline{X})^{K} \simeq \bigvee_{j=k+2}^{m}\left(\bigvee_{1 \leq i_{1}<\cdots<i_{j} \leq m}\left(\begin{array}{l}
j-1 \\
k+1
\end{array}\right) \Sigma^{k+1} X_{i_{1}} \wedge \cdots \wedge X_{i_{j}}\right) .
$$

In particular, if $K=\Delta_{0}^{m-1}$ then $K$ is $m$ disjoint points, and there is a homotopy equivalence

$$
(\underline{C X}, \underline{X})^{K} \simeq \bigvee_{j=2}^{m}\left(\bigvee_{1 \leq i_{1}<\cdots<i_{j} \leq m}(j-1) \Sigma X_{i_{1}} \wedge \cdots \wedge X_{i_{j}}\right) .
$$

Consequently, if $K$ is $m$ disjoint points then there is a homotopy equivalence

$$
\mathcal{Z}_{K} \simeq \bigvee_{k=2}^{n}(k-1)\left(\begin{array}{l}
n \\
k
\end{array}\right) S^{k+1}
$$

a case revisted in $([\mathrm{GT} 1])$.

There are many shifted simplicial complexes which are not the full skeleta of simplices. For example, let $K$ be the simplicial complex with vertices $\{1,2,3,4\}$ and edges $\{(1,2),(1,3),(1,4),(2,3),(2,4)\}$ :

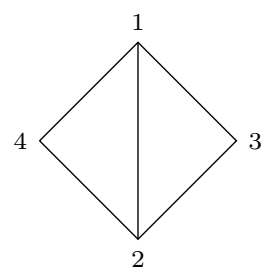

In [GT3] a detailed description is given of how (12) and Lemma (5.1) can be applied iteratively to show that there is a homotopy equivalence

$$
(\underline{C X}, \underline{X})^{K} \simeq\left(\Sigma X_{3} \wedge X_{4}\right) \vee\left(\Sigma^{2} X_{1} \wedge X_{2} \wedge X_{3}\right) \vee\left(\Sigma^{2} X_{1} \wedge X_{2} \wedge X_{4}\right) \vee 2 \cdot\left(\Sigma^{2} X_{1} \wedge X_{2} \wedge X_{3} \wedge X_{4}\right)
$$

In particular, in the moment-angle complex case there is a homotopy equivalence

$$
\mathcal{Z}_{K} \simeq S^{3} \vee 2 S^{5} \vee 2 S^{6}
$$

\section{The hомотору type of $\mathcal{Z}_{K}$ And $(\underline{C X}, \underline{X})^{K}$ : PART II}

Ideally, one would like to push Theorem 5.4 as far as possible by identifying the maximal class of simplicial complexes for which Bahri, Bendersky, Cohen and Gitler's decomposition of $\Sigma(\underline{C X}, \underline{X})^{K}$ desuspends. To find such a family, we go back to moment-angle complexes and take our cue from combinatorics and commutative algebra. 
Recall from Section 1.2 that $H^{*}\left(\mathcal{Z}_{K}\right) \cong \operatorname{Tor}_{\mathbb{Z}\left[v_{1}, \ldots, v_{m}\right]}(\mathbb{Z}[K], \mathbb{Z})$ and that the Stanley-Reisner ring $\mathbf{k}[K]$ is Golod over a field $\mathbf{k}$ if all cup products and higher Massey products in $\operatorname{Tor}_{\mathbf{k}\left[v_{1}, \ldots, v_{m}\right]}(\mathbf{k}[K], \mathbf{k})$ are trivial. In fact, Berglund and Jöllenbeck [BJ] showed that the triviality of the higher Massey products follows from the triviality of cup products, so we may as well only consider cup products. If $\mathcal{Z}_{K}$ is homotopy equivalent to a wedge of spheres then all cup products in $H^{*}\left(\mathcal{Z}_{K}\right)$ vanish so $\mathbb{Z}[K]$ is Golod over any field. The converse does not hold as there are examples of Golod $\mathcal{Z}_{K}$ with torsion (an example will be discuss later in Section 7). For many years combinatorists and commutative algebraists have identified classes $\mathcal{K}$ of simplicial complexes which have the property that if $K \in \mathcal{K}$ then $\mathbb{Z}[K]$ is Golod over any field $\mathbf{k}$, implying that $H^{*}\left(\mathcal{Z}_{K} ; \mathbf{k}\right)$ has no cup products or higher Massey products. One such class is shifted complexes, others are shellable and sequentially Cohen-Macaulay complexes (to be discussed momentarily). In all these cases $K_{I}$ for $I \notin K$ is homotopy equivalent to a wedge of spheres. It is natural to ask whether in these cases $\mathcal{Z}_{K}$ is homotopy equivalent to a wedge of spheres. If $\mathbf{k}$ is any field, one might hope that there is a a maximal class $\mathcal{K}$ of simplicial complexes with torsion free cohomology which produces three equivalent statements:

- $K \in \mathcal{K}$;

- $\mathbf{k}[K]$ is Golod;

- $\mathcal{Z}_{K}$ is homotopy equivalent to a wedge of spheres.

Proving implications between these statements has been a prime motivator in the area for the past few years. In this section we will outline three directions where progress has been made.

6.1. Vertex-decomposable, shellable and sequentially Cohen-Macaulay complexes. It is helpful to have candidates for the class $\mathcal{K}$. As summarised in [BW], there is a hierarchy of families of simplicial complexes:

$$
\text { shifted } \subset \text { vertex decomposable } \subset \text { shellable } \subset \text { sequentially Cohen-Macaulay }
$$

where the containments in each case are proper. The shifted case was discussed in the previous section.

Definition 6.1. A simplicial complex $K$ is vertex-decomposable if:

(i) $K$ is a simplex or $K=\emptyset$, or

(ii) there exists a vertex $v \in K$ such that:

(a) $K \backslash v$ and $\operatorname{link}_{K}(v)$ are vertex-decomposable;

(b) no facet of $\operatorname{link}_{K}(v)$ is a facet of $K \backslash v$.

The vertex-decomposable case is similar in spirit to the shifted case in the sense that it is described in terms of the vertices of the simplicial complex and is well suited to an inductive procedure using Lemma 5.1 and (12) since both $\operatorname{link}_{K}(v)$ and $K \backslash\{v\}$ are also vertex-decomposable. 
Grujić and Welker [GW] used combinatorial Morse theory and the general strategy of Lemma 5.1 and (12) to show the following. The statement is phrased in terms of the dual of a simplicial complex. The Alexander dual of a simplicial complex $K$ on the index set $[m]$ is the simplicial complex

$$
K^{\circ}=\{\sigma \subseteq[m] \mid[m] \backslash \sigma \notin K\}
$$

Theorem 6.2. Suppose that $K$ is a simplicial complex whose Alexander dual is vertex-decomposable. Then $\left(\underline{D^{n}}, \underline{S^{n-1}}\right)^{K} \in \mathcal{W}_{m}$.

Note that no mention was made of the Alexander dual in the corresponding statement for shifted complexes in Theorem 5.3. This stems from the fact that shifted complexes are self-dual. Analysing Grujić and Welker's approach, it is clear that it is really better to think in terms of the dual. Another point to note about Theorem 6.2 is that it is specialised to the case of polyhedral products on pairs $\left(\underline{D^{n}}, \underline{S^{n-1}}\right)$. This is due to the Morse theoretic methods used. Ideally, there should be a more general statement that covers all polyhedral products $(\underline{C X}, \underline{X})^{K}$.

However, instead of pursuing this, Iriye and Kishimoto [IK2] made the jump to shellable and sequentially Cohen-Macaulay simplicial complexes. We give only the definition of a shellable complex to illustrate a point. A facet of a simplicial complex is a maximal simplex and a simplicial complex is pure if all facets have the same dimension.

Definition 6.3. A simplicial complex $K$ is shellable if there is an ordering of the facets $F_{1}, \ldots, F_{t}$ such that

$$
\left\langle F_{k}\right\rangle \cap\left\langle F_{1}, \ldots, F_{k-1}\right\rangle
$$

is pure and $\left(\operatorname{dim}\left(F_{k}\right)-2\right)$-dimensional for $k=2, \ldots, t$, where \langle\rangle denotes the linear span.

A simplicial complex is Cohen-Macaulay over $\mathbb{Z}$ if its Stanley-Reisner ring $\mathbb{Z}[K]$ is a CohenMacaulay ring, meaning that the Krull dimension and the depth of $\mathbb{Z}[K]$ are equal. One property of Cohen-Macaulay complexes is that they are pure. A non-pure generalisation is given by sequentially Cohen-Macaulay complexes. To define these, we follow [BWW]. If $K$ is a simplicial complex and $i \geq 0$, let $K^{\langle i\rangle}$ be the subcomplex of $K$ generated by the faces of dimension $\geq i$. The simplicial complex $K$ is sequentially acyclic over $\mathbb{Z}$ if $K^{\langle i\rangle}$ has the property that $\widetilde{H}_{r}\left(K^{\langle i\rangle}\right)=0$ for all $r<i \leq$ $\operatorname{dim} K$. The link of a face $\sigma$ in $K$ is the subcomplex

$$
\operatorname{link}_{K}(\sigma)=\{\tau \in K \mid \sigma \cup \tau \in K, \sigma \cap \tau=\emptyset\}
$$

Definition 6.4. A simplicial complex $K$ is sequentially Cohen-Macaulay over $\mathbb{Z}$ if $\operatorname{link}_{K}(\sigma)$ is sequentially acyclic over $\mathbb{Z}$ for all $\sigma \in K$.

Observe that the definitions of shellable and sequentially Cohen-Macaulay complexes are very different in spirit to that of shifted or vertex-decomposable simplicial complexes, in the sense that 
the definitions are not in terms of the vertices. So a different approach is needed for studying the corresponding polyhedral products.

Iriye and Kishimoto [IK2] give a "fat wedge filtration" of the polyhedral product, which is governed by the fat wedge filtration of the real moment-angle complex $\left(\underline{D^{1}}, \underline{S^{0}}\right)^{K}$. They show that if this filtration satisfies a triviality condition then the Bahri-Bendersky-Cohen-Gitler decomposition of $\Sigma(\underline{C X}, \underline{X})^{K}$ desuspends. They then identify a family of simplicial complexes that satisfy this triviality condition.

Definition 6.5. A simplicial complex $K$ is fillable if there are minimal non-faces $M_{1}, \ldots, M_{r}$ of $K$ such that $\left|K \cup M_{1} \cup \cdots \cup M_{r}\right|$ is contractible.

They show that if every full subcomplex $K_{I}$ of $K$ is fillable then the fat wedge filtration of $K$ is trivial and so the Bahri-Benersky-Cohen-Gitler decomposition of $\Sigma(\underline{C X}, \underline{X})^{K}$ desuspends. They go on to show that if the Alexander dual of $K$ is shellable then $K$ is fillable, and hence $(\underline{C X}, \underline{X})^{K} \in \mathcal{W}_{m}$.

To deal with the sequentially Cohen-Macaulay case they generalise the notion of fillable to homology fillable, show that this also implies the triviality of the fat wedge filtration, and show that the Alexander dual of a sequentially Cohen-Macaulay complex is homology fillable. Collecting these results gives the following.

Theorem 6.6. Let $K$ be a simplicial complex on the vertex set $[m]$ whose Alexander dual is shellable or sequentially Cohen-Macaulay (or more generally, $K$ is fillable or homology fillable). Then the Bahri-Bendersky-Cohen-Gitler decomposition of $\Sigma(\underline{C X}, \underline{X})^{K}$ desuspends, implying that

$$
\Sigma(\underline{C X}, \underline{X})^{K} \in \mathcal{W}_{m}
$$

Consequently, $\mathcal{Z}_{K}$ is homotopy equivalent to a wedge of spheres.

6.2. Configuration spaces and polyhedral products. Theorem 6.6 is inspired by known results in combinatorial commutative algebra. By [HRW] every sequentially Cohen-Macaulay simplicial complex $K$ has the property that $\mathbb{Z}[K]$ is Golod over every field. So it is natural to try to show that if $K$ is sequentially Cohen-Macaulay then $\mathcal{Z}_{K}$ is homotopy equivalent to a wedge of spheres. Along the way one discovers that the method used to prove this actually holds for a larger family of complexes, the homology fillable complexes.

Alternatively, it may be fruitful to work with the Golod condition more directly. Recall that the Stanley-Reisner face ring $\mathbb{Z}[K]$ is Golod if all cup products and higher Massey products in $H^{*}\left(\mathcal{Z}_{K}\right) \cong \operatorname{Tor}_{K\left[v_{1}, \ldots, v_{m}\right]}(\mathbb{Z}[K], \mathbb{Z})$ vanish. All cup products and higher Massey products in $H^{*}\left(\mathcal{Z}_{K}\right)$ also vanish if $\mathcal{Z}_{K}$ is homotopy equivalent to a wedge of spheres. But there are known examples (see the end of Section 7 ) where $\mathbb{Z}[K]$ is Golod but $\mathcal{Z}_{K}$ is not homotopy equivalent to a wedge of spheres. So it is too strong to ask that $\mathcal{Z}_{K}$ be homotopy equivalent to a wedge of spheres. Considering a desuspension of (6), we see that $\mathcal{Z}_{K}$ might have summands of the form $\Sigma^{|I|}\left|K_{I}\right|$ which can in general 
be any suspension space. Therefore a sensible alternative arises if the problem is considered more abstractly. Any co- $H$ space $Y$ has the property that all cup products and higher Massey products in $H^{*}(Y)$ vanish. A more reasonable question to ask is therefore: if $\mathbb{Z}[K]$ is Golod is $\mathcal{Z}_{K}$ a co- $H$-space? Are the two notions equivalent?

Resolving these questions is the motivation behind a recent paper by Beben and the first author $[\mathrm{BG}]$. They constructed a configuration space model for certain mapping spaces. To keep focused on the output we care most about in this paper, we consider a special case of their more general construction. Let $W=(\underline{X}, \underline{A})^{K}$, a subspace of $\prod_{i=1}^{m} X_{i}$. For $\ell \geq 0$, let

$$
W^{\ell}=\left(\underline{\Sigma^{\ell} X}, \underline{\Sigma^{\ell} A}\right)^{K}
$$

be a coordinate suspension space of $W$. For example, if $W=\left(D^{1}, S^{0}\right)^{K}$ then $W^{1} \simeq\left(D^{2}, S^{1}\right)^{K}=\mathcal{Z}_{K}$. They then consider the mapping space

$$
\operatorname{map}\left((Y, B) ; W^{\ell}\right)
$$

whose elements consist of maps $f: Y \longrightarrow W^{\ell}$ with the property that $B \subseteq Y$ is sent to the basepoint. Note here that $B$ may be empty, in which case one obtains a model for the space of free maps from $Y$ to $W^{\ell}$. They show that for certain pairs $(Y, B)$, which include the cases $\left(D^{n}, S^{n-1}\right)$, that $\operatorname{map}\left((Y, B) ; W^{\ell}\right)$ is homotopy equivalent to a certain configuration space of labelled particles.

An important special case is when $(Y, B)=\left(S^{1}, *\right)$, in which case one obtains a configuration space model for $\operatorname{map}\left(\left(S^{1}, *\right) ; W^{\ell}\right)=\Omega W^{\ell}$. The model is then used to produce a homotopy decomposition of $\Sigma \Omega W^{\ell}$ whose wedge summands correspond to quotient labelled configuration spaces, whose properties are determined in part - but not exclusively - by the underlying simplicial complex $K$.

Specialising to $W=\left(D^{1}, S^{0}\right)^{K}$, in which case a model is obtained for $\Omega W^{1} \simeq \Omega \mathcal{Z}_{K}$, and a homotopy decomposition is obtained for $\Sigma \Omega \mathcal{Z}_{K}$. The goal, remember, is to say something about $\mathbb{Z}[K]$ being Golod implying that $\mathcal{Z}_{K}$ is a co- $H$-space. It is well known that a space $Y$ is a co- $H$-space if and only if $Y$ retracts off $\Sigma \Omega Y$. So the aim is to show that if $\mathbb{Z}[K]$ is Golod then the configuration space model ensures that $\mathcal{Z}_{K}$ retracts off $\Sigma \Omega \mathcal{Z}_{K}$. In practise, the Golod condition is difficult to work with so a variation of it is defined, called homotopy Golod, which tries to capture the notion of the cup product and all higher Massey products being trivial in $H^{*}\left(\mathcal{Z}_{K}\right)$ from a homotopy theoretic point of view. The definition, which follows, now, is defined directly on the simplicial complex rather than the Stanley-Reisner ring.

Definition 6.7. A simplicial complex $K$ on the vertex set $[m]$ is homotopy Golod if $K$ is a single vertex, or recursively, if $K \backslash\{i\}$ is homotopy Golod for each $i \in[m]$ and there is a basepoint preserving map

$$
\Psi_{K}: \Sigma^{m}|K| \longrightarrow \Sigma\left|\Delta^{m-1}\right|
$$


such that for any $y=\left(t_{1}, \ldots, t_{m-1}\right) \in\left(\prod_{i=1}^{m}[-1,1]\right) / \partial\left(\prod_{i=1}^{m}[-1,1]\right) \cong S^{m-1}$ that is not the basepoint, $\Psi_{K}$ maps the subspace $\{y\} \wedge \Sigma|K|$ of $\Sigma^{m}|K|$ as follows:

(1) when $t_{1}=\cdots=t_{m-1}=0$, the restriction of $\Psi_{K}$ to $\{y\} \wedge \Sigma|K|$ is the suspension of the inclusion $\Sigma|K| \longrightarrow \Sigma\left|\Delta^{m-1}\right|$

(2) letting $S_{y}=\left(t_{1}, \ldots, t_{n-1}, 0\right)$ and $\left(I_{1}, \ldots, I_{n}\right)$ be an ordered partition of $[m]$ that depends on $S_{y}, \Psi_{K}$ maps $\{y\} \wedge \Sigma|K|$ to a subspace of $\Sigma\left|K_{I_{1}} * \cdots * K_{I_{m}}\right| \subseteq \Sigma\left|\Delta^{m-1}\right|$.

Two additional equivalent definitions of homotopy Golod are given in [BG], and they show that the class of homotopy Golod simplicial complexes includes those whose Alexander duals are sequentially Cohen-Macaulay. Beben and the first author also give an example to show that this class is strictly larger than the class of sequentially Cohen-Macaulay complexes. They prove the following.

Theorem 6.8. Let $K$ be a simplicial complex which is homotopy Golod. Then $\mathcal{Z}_{K}$ is a co-H-space.

Theorem 6.8 implies that a homotopy Golod complex $K$ has trivial cup products and higher Massey products in $H^{*}\left(\mathcal{Z}_{K}\right)$, and so $\mathbb{Z}[K]$ is Golod. It is not known if $\mathbb{Z}[K]$ is Golod then $K$ is homotopy Golod. Also, it is not known if the converse of Theorem 6.8 holds, that is, whether $\mathcal{Z}_{K}$ being a co- $H$-space implies that $K$ is homotopy Golod. However, Beben and the first author go on to show that a weaker version of the homotopy Golod property does establish an equivalence.

Let $\Delta_{m}$ be the diagonal

$$
\Delta_{m}=\left\{\left(x_{1}, \ldots, x_{n}\right) \in \mathbb{R}^{m} \mid x_{1}=\cdots=x_{m}\right\} .
$$

Let $\mathcal{Q}_{K}$ be the subspace of the half-smash product $\left.\left(\left(\mathbb{R}^{m}-\Delta_{m}\right) \times \Sigma\left|\Delta^{m-1}\right|\right) /\left(\mathbb{R}^{m}-\Delta_{m}\right) \times *\right)$ defined as follows:

$$
\mathcal{Q}_{K}=\bigcup_{\substack{y \in\left(\mathbb{R}^{m}-\Delta_{m}\right) \\\left(I_{1}, \ldots, I_{n}\right)=[m]_{y}}}\{y\} \wedge \Sigma\left|K_{I_{1}} * \cdots * K_{I_{n}}\right|
$$

where $\left(I_{1}, \ldots, I_{n}\right)=[m]_{y}$ of $[m]$ depending on $y$.

Definition 6.9. A simplicial complex $K$ on the vertex set $[m]$ is weakly homotopy Golod if $K$ is a single vertex, or recursively, if $K \backslash\{i\}$ is weakly homotopy Golod for each $i \in[m]$ and the map

$$
\Phi_{K}: \Sigma^{m}|K| \longrightarrow \Sigma \mathcal{Q}_{K}
$$

given for any $z \in|K|, t_{1}, \ldots, t_{m-1}, t \in[-1,1]$ and $\beta=\max \left(\left|t_{1}\right|, \cdots,\left|t_{m-1}\right|, 0\right)$ by

$$
\Phi_{K}\left(t_{1}, \ldots, t_{m-1}, t, z\right)=\left(2 \beta-1,\left(t_{1}, \ldots, t_{m-1}, 0\right),(t, z)\right)
$$

is null homotopic.

Theorem 6.10. The simplicial complex $K$ is weakly homotopy Golod if and only if $\mathcal{Z}_{K}$ is a co-Hspace. 
Theorem 6.10 classifies those simplicial complexes $K$ for which $\mathcal{Z}_{K}$ is a co- $H$-space. However, it remains open as to whether $K$ being weakly homotopy Golod is equivalent to $\mathbb{Z}[K]$ being Golod.

6.3. Gluing along a common face. To this point the families of simplicial complexes which have produced decompositions $(\underline{C X}, \underline{X})^{K} \in \mathcal{W}_{m}$ have all come from combinatorics - shifted, shellable, sequentially Cohen-Macaulay - or from generalisations aimed at establishing these cases - fillable, homology fillable, homotopy Golod. There is another operation on simplicial complexes which does not behave well combinatorially but does behave well with polyhedral products: gluing two simplicial complexes along a common face.

Let $K$ be a simplicial complex on the vertex set $[m]$. Suppose that $K=K_{1} \cup_{\tau} K_{2}$ where $\tau$ is a simplex in $K$. That is, there is a pushout of simplicial complexes

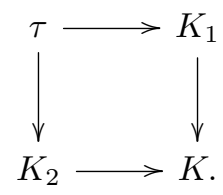

Geometrically, $|K|$ is the result of gluing $\left|K_{1}\right|$ and $\left|K_{2}\right|$ together along a common face. Relabelling the vertices if necessary, we may assume that $K_{1}$ is defined on the vertices $\{1, \ldots, \ell\}, K_{2}$ is defined on the vertices $\{\ell-k+1, \ldots, m\}$ and $\tau$ is defined on the vertices $\{\ell-k+1, \ldots, \ell\}$. Let $\bar{K}_{1}, \bar{K}_{2}$ and $\bar{\tau}$ be $K_{1}, K_{2}$ and $\tau$ regarded as simplicial complexes on [m]. So $K=\bar{K}_{1} \cup_{\bar{\tau}} \bar{K}_{2}$.

Let $\sigma \in K_{1}$ and let $\bar{\sigma}$ be its image in $\bar{K}_{1}$. By definition of $\bar{\sigma}$, we have $i \notin \bar{\sigma}$ for $i \in\{\ell+1, \ldots, m\}$. Thus $(\underline{C X}, \underline{X})^{\bar{\sigma}}=(\underline{C X}, \underline{X})^{\sigma} \times X_{\ell+1} \times \cdots \times X_{m}$. Consequently, taking the union over all the faces in $\bar{K}_{1}$, we obtain

$$
(\underline{C X}, \underline{X})^{\bar{K}_{1}}=(\underline{C X}, \underline{X})^{K_{1}} \times X_{\ell+1} \times \cdots \times X_{m}
$$

Similarly, we have

$$
(\underline{C X}, \underline{X})^{\bar{K}_{2}}=X_{1} \times \cdots \times X_{\ell-k} \times(\underline{C X}, \underline{X})^{K_{2}} .
$$

Since $\tau=\Delta^{k-1}$, we have $(\underline{C X}, \underline{X})^{\tau}=C X_{\ell-k+1} \times \cdots \times C X_{\ell}$, so as above we obtain

$$
(\underline{C X}, \underline{X})^{\bar{\tau}}=X_{1} \times \cdots \times X_{\ell-k} \times C X_{\ell-k+1} \times \cdots \times C X_{\ell} \times X_{\ell+1} \times \cdots \times X_{m} .
$$

Let $M=X_{1} \times \cdots \times X_{\ell-k}$ and $N=X_{\ell+1} \times \cdots \times X_{m}$. By applying polyhedral products to (14), we obtain a pushout

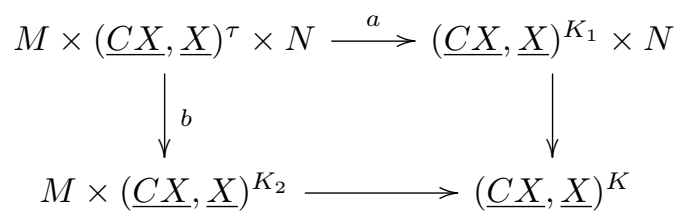

where $a$ and $b$ are defined coordinate-wise. Since $\tau$ is a simplex, $(\underline{C X}, \underline{X})^{\tau}$ is a product of cones and so is contractible. The definitions of the maps immediately implies that $a$ restricted to $N$ is the identity map and similarly for $b$ restricted to $M$. In [GT3] a straightforward lemma is proved 
to show that $a$ restricted to $M$ is null homotopic and similarly for $b$ restricted to $N$. The homotopy type of $(\underline{C X}, \underline{X})^{K}$ is then identified by a general lemma, also proved in [GT3].

Lemma 6.11. Let

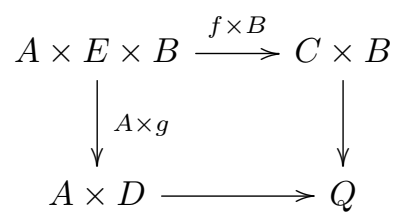

be a homotopy pushout, where $E$ is contractible and $f$ and $g$ are null homotopic. Then there is a homotopy equivalence

$$
Q \simeq(A * B) \vee(A \ltimes D) \vee(C \rtimes B)
$$

Applying Lemma 6.11 to the pushout in (15), we obtain the following.

Theorem 6.12. Let $K$ be a simplicial complex on the vertex set $[m]$. Suppose that $K=K_{1} \cup_{\tau} K_{2}$ where $\tau$ is a common face of $K_{1}$ and $K_{2}$. Then there is a homotopy equivalence

$$
(\underline{C X}, \underline{X})^{K} \simeq(M * N) \vee\left((\underline{C X}, \underline{X})^{K_{1}} \rtimes N\right) \vee\left(M \ltimes(\underline{C X}, \underline{X})^{K_{2}}\right)
$$

where $M=X_{1} \times \cdots \times X_{\ell-k}$ and $N=X_{\ell+1} \times \cdots \times X_{m}$.

Notice that $M * N \in \mathcal{W}_{m}$. If $(\underline{C X}, \underline{X})^{K_{1}} \in \mathcal{W}_{m}$ then it is a suspension, implying that

$$
(\underline{C X}, \underline{X})^{K_{1}} \rtimes N \simeq(\underline{C X}, \underline{X})^{K_{1}} \vee\left((\underline{C X}, \underline{X})^{K_{1}} \wedge N\right) \in \mathcal{W}_{m} .
$$

Similarly, if $(\underline{C X}, \underline{X})^{K_{2}} \in \mathcal{W}_{m}$ then $(\underline{C X}, \underline{X})^{K_{2}} \ltimes N \in \mathcal{W}_{m}$. We obtain the following corollary.

Corollary 6.13. With hypotheses as in Theorem 6.12, if $(\underline{C X}, \underline{X})^{K_{1}}$ and $(\underline{C X}, \underline{X})^{K_{2}}$ are in $\mathcal{W}_{m}$ then so is $(\underline{C X}, \underline{X})^{K}$.

As all what we need is that $(\underline{C X}, \underline{X})^{K_{1}}$ and $(\underline{C X}, \underline{X})^{K_{1}}$ are suspension spaces, we can rephrase Corollary 6.13 in much more general setting.

Corollary 6.14. With hypotheses as in Theorem 6.12, if the stable splittings (5) for $(\underline{C X}, \underline{X})^{K_{1}}$ and $(\underline{C X}, \underline{X})^{K_{2}}$ desuspend, then the one for $(\underline{C X}, \underline{X})^{K}$ does as well.

In particular, Corollary 6.13 holds if $K_{1}$ and $K_{2}$ are shifted, shellable or sequentially CohenMacaulay. Note that $K$ need not be shifted, shellable or sequentially Cohen-Macaulay. On the other hand, $K$ is homology fillable and homotopy Golod in each case, so Corollary 6.13 does not strictly extend the family of simplicial complexes for which $(\underline{C X}, \underline{X})^{K}$ is now known to be in $\mathcal{W}_{m}$. Nevertheless, Corollary 6.13 is a simple, practical means of producing interesting examples of simplicial complexes with $(\underline{C X}, \underline{X})^{K} \in \mathcal{W}_{m}$.

In the terms of Golod complexes, looking combinatorially on all possible cup products, Limonchenko [Li] produced an analogues result. 
Proposition 6.15. With hypotheses as in Theorem 6.12, if $K_{1}$ and $K_{2}$ are Golod complexes, then so is $K$.

One very interesting example is flag complexes. A simplicial complex $K$ is a flag complex if each of its missing faces has two vertices. Equivalently, $K$ is flag if any set of vertices of $K$ which are pairwise connected by edges spans a simplex. In [GPTW] it is shown that if $K$ is Golod flag then there is an ordering $I_{1}, \ldots, I_{s}$ of its maximal faces such that $\left(\bigcup_{j<k} I_{j}\right) \cap I_{k}$ is a simplex for each $k=1, \ldots, s$. That is, $K$ can be formed by iteratively gluing on simplices along common faces. Therefore, if $K$ is flag then by iterating Corollary 6.13 we obtain $(\underline{C X}, \underline{X})^{K} \in \mathcal{W}_{m}$. In particular, the moment-angle complex $\mathcal{Z}_{K}$ is homotopy equivalent to a wedge of spheres.

The flag case was pursued more deeply in [GPTW] to show that the notional connection between Golod rings and $\mathcal{Z}_{K}$ being homotopy equivalent to a wedge of spheres can be made precise.

Theorem 6.16. Let $K$ be a flag complex and $\mathbf{k}$ a field. The following conditions are equivalent:

(a) $\mathbf{k}[K]$ is a Golod ring;

(b) the multiplication in $H^{*}\left(\mathcal{Z}_{K}\right)$ is trivial;

(c) $\mathcal{Z}_{K}$ is homotopy equivalent to a wedge of spheres.

Another application of Corollary 6.13 arose in [GT3] in the context of the simplicial wedge construction. Let $K$ be a simplicial complex on vertices $\left\{v_{1}, \ldots, v_{m}\right\}$. Fix a vertex $v_{i}$. By doubling the vertex $v_{i}$, define a new simplicial complex $K\left(v_{i}\right)$ on the $m+1$ vertices $\left\{v_{1}, \ldots, v_{i-1}, v_{i, 1}, v_{i, 2}, v_{i+1}, \ldots, v_{m}\right\}$ by

$$
K\left(v_{i}\right)=\left(v_{i, 1}, v_{i, 2}\right) * \operatorname{link}_{K}\left(v_{i}\right) \cup\left\{v_{i, 1}, v_{i, 2}\right\} * K \backslash\left\{v_{i}\right\}
$$

where $\left(v_{i, 1}, v_{i, 2}\right)$ denotes the one dimensional simplex on the vertices $v_{i, 1}$ and $v_{i, 2}$. The simplicial complex $K\left(v_{i}\right)$ is called the simplicial wedge of $K$ on $v_{i}$. This combinatorial construction reflects the process of polarising the Stanley-Reisner ring of a multicomplex, which needs not be square free as introduced by Trevisan $[\operatorname{Tr}]$. Let $J=\left(j_{1}, \ldots, j_{m}\right)$ be an $n$ tuple of positive integers, and let $n=\sum_{i=1}^{m} j_{i}$. The simplicial multiwedge $K(J)$ is the new simplicial complex on $n$-vertices

$$
\left\{v_{1,1}, \ldots, v_{1, j_{1}}, v_{2,1}, \ldots, v_{2, j_{2}}, \ldots, v_{m, 1}, \ldots, v_{m, j_{m}}\right\}
$$

obtained by iteratively applying the simplicial wedge construction, starting with $K$. It turns out that the order in which the simplicial wedge construction is iterated does not affect the complex produced, so $K(J)$ is well-defined. Let $\underline{X}=\left(X_{1}, \ldots, X_{n}\right)$ be $n$ topological spaces. Let $n_{0}=0$ and for $1 \leq i \leq m$, let $n_{i}=\Sigma_{k=1}^{i} j_{k}$. Note that $n_{m}=n$. The $n$ topological spaces $X_{1}, \ldots, X_{n}$ are then written as

$$
X_{n_{0}+1}, \ldots, X_{n_{1}}, X_{n_{1}+1}, \ldots, X_{n_{2}}, X_{n_{2}+1}, \ldots, X_{n_{m-1}}, X_{n_{m-1}+1}, \ldots X_{n_{m}}
$$


Define

$$
\left(\underline{C\left(*_{J} X\right)}, \underline{*_{J} X}\right)=\left\{\left(C\left(X_{n_{i-1}+1} * \ldots * X_{n_{i}}\right), X_{n_{i-1}+1} * \ldots * X_{n_{i}}\right)\right\}_{i=1}^{m} .
$$

In [GT3] the following was proved, with the consequence now following from Theorems 6.6 and 6.8.

Theorem 6.17. For a simplicial complex $K$ on $m$-vertices, an $m$-tuple $J=\left(j_{1}, \ldots, j_{m}\right)$ of positive integers and $\sum_{i=1}^{m} j_{i}$ topological pairs $\left(C X_{i}, X_{i}\right)$ where $X_{i}$ is a finite $C W$-complex, there is a homeomorphism of polyhedral products

$$
\left(\underline{C\left(*_{J} X\right)}, \underline{*_{J} X}\right)^{K} \longrightarrow(\underline{C X}, \underline{X})^{K(J)} .
$$

Consequently, if $K$ is homology fillable or homotopy Golod then

$$
(\underline{C X}, \underline{X})^{K(J)} \simeq\left(\bigvee_{I \notin K}\left|K_{I}\right| *{\widehat{\left(*_{J} X\right)}}^{I}\right) .
$$

\section{Minimally NON-Golod COMPlexes And CONneCted sums of PRODUCts of TwO SPHERES}

There are now well established connections between a Stanley-Reisner face ring $\mathbf{k}[K]$ being Golod for any field $\mathbf{k}$ and the corresponding moment-angle complex $\mathcal{Z}_{K}$ being homotopy equivalent to a wedge of spheres. One would like to take this to the next level.

A simplicial complex $K$ is called Golod over a field $\mathbf{k}$ if its Stanley-Reisner face ring $\mathbf{k}[K]$ is Golod. The following definition is due to Berglund and Jöllenbeck [BJ].

Definition 7.1. Let $K$ be a simplicial complex on $m$ vertices. For $1 \leq i \leq m$, let $I_{i}=[m] \backslash\{i\}$. Then $K$ is minimally non-Golod if every full subcomplex $K_{I_{i}}$ is Golod.

An important family of examples of minimally non-Golod simplicial complexes is given by vertex cutting operations on simple polytopes. A (convex) polytope is the convex hull of a finite set of points in $\mathbb{R}^{n}$. Its dimension is the dimension of its affine hull. Let $P$ be a $d$-dimensional polytope. A facet of $P$ is a $(d-1)$-dimensional face. The polytope $P$ is simple if each vertex lies in exactly $d$ facets of $P$. A partial ordering may be defined on the faces of $P$ by inclusion. This determines a poset called the face poset of $P$. The opposite poset, given by reversing the order, determines another polytope $P^{*}$ called the dual of $P$. If $P$ is simple then $P^{*}$ is a simplicial complex. Let $\partial P$ be the boundary of $P$.

Definition 7.2. Let $P$ be a simple polytope of dimension $d$ and let $V(P)$ be its vertex set. A hyperplane $H$ in $\mathbb{R}^{d}$ cuts a vertex $x$ of $P$ if $x$ and $V(P) /\{x\}$ lie in different open half-spaces of $H$. Let $Q$ be the intersection of $P$ with the closed half-space of $H$ containing $V(P) /\{x\}$. We say that $Q$ is obtained from $P$ by a vertex cut operation. 
Diagrammatically, this is pictured as follows:

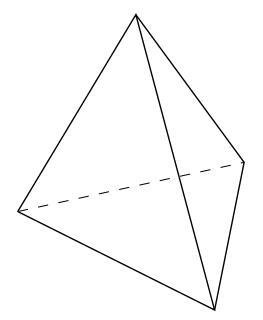

$P$

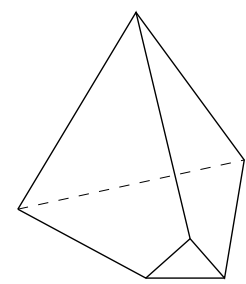

$Q$

In particular, starting from $\Delta^{2}$, iteratively cutting off $n$ vertices gives an $(n+3)$-gon. Berglund and Jöllenbeck [BJ] showed that if $P$ is a simple polytope that has been obtained from the $d$-simplex by iteratively cutting off vertices then, regardless of the order in which the vertices were cut, the simplicial complex $\partial P^{*}$ is minimally non-Golod.

Turning to topology (and geometry), again assume that $P$ is a simple polytope that has been obtained from $\Delta^{d}$ by iteratively cutting off $\ell-1$ vertices. Bosio and Meersseman [BM], crediting an earlier construction of MacGavran [M], showed that there is a diffeomorphism

$$
\mathcal{Z}_{\partial P^{*}} \cong \#_{k=3}^{\ell+1}\left(S^{k} \times S^{\ell+2 d-k}\right)^{\#(k-2)\left(\begin{array}{c}
\ell \\
k-1
\end{array}\right)}
$$

where $\left(S^{k} \times S^{\ell+2 d-k}\right)^{\# n}$ is the $n$-fold connected sum of $S^{k} \times S^{\ell+2 d-k}$ with itself. Gitler and Lopez de Medrano [GL] generalized this to show that for such polytopes $P$, if $K=\partial P^{*}$ then for any sequence $J=\left(j_{1}, \ldots, j_{m}\right)$ the simplicial multiwedge $K(J)$ has the property that it is diffeomorphic to a connected sum of products of two spheres.

This suggests there is a correspondence analogous to that for $K$ being Golod and $\mathcal{Z}_{K}$ being homotopy equivalent to a wedge of spheres:

- $K$ is minimally non-Golod;

- $\mathcal{Z}_{K}$ is diffeomorphic to a connected sum of products of two spheres.

Some initial results on this problem have been obtained but much remains to be done. We give a brief outline of some of what has been done.

Since Golod and minimally non-Golod properties are expressed in homotopy theoretical properties of $\mathcal{Z}_{K}$, one might look at connected sums from a homotopy theoretical point of view as a space where only one top cell is attached. Let $X$ and $Y$ be topological spaces with one top $n$-dimensional cell attached by $\alpha_{X}$ and $\alpha_{Y}$ respectively. Then the connected sum $X \# Y$ of $X$ and $Y$ is the cofibre of the map

$$
S^{n-1} \stackrel{\alpha_{X}+\alpha_{Y}}{\longrightarrow} \bar{X} \vee \bar{Y}
$$

where $\bar{X}$ and $\bar{Y}$ are $(n-1)$-skeletons of $X$ and $Y$, respectively.

Then more general correspondence between combinatorics and topology could be stated in the following way. 
- $K$ is minimally non-Golod;

- $\mathcal{Z}_{K}$ is homotopic to a connected sum of $X$ and $Y$, where $\bar{X}$ and $\bar{Y}$ are Golod spaces.

When $K$ is a flag complex an exact correspondence holds [GPTW].

Theorem 7.3. Assume that $K$ is flag and $\mathbf{k}$ is a field. The following conditions are equivalent:

(a) $K$ is minimally non-Golod;

(b) $\mathcal{Z}_{K}$ is homeomorphic to a connected sum of products of two spheres.

In fact, if $K$ is flag and minimally non-Golod then it is the boundary of an $n$-gon with $n \geq 4$. Bosio and Meersseman [BM], or MacGavran [M], then say that $\mathcal{Z}_{K}$ is diffeomorphic to a connected sum of products of two spheres.

In [T] a connection is made between the homotopy type of $\mathcal{Z}_{K}$ when $K$ is $\ell$ disjoint points and the diffeomorphism type of $\mathcal{Z}_{K}$ when $K=\partial P^{*}$ for $P$ a simple polytope that has been obtained from $\Delta^{d}$ by $\ell-1$ vertex cuts. When $K$ is $\ell$ disjoint points, by (13) we obtain

$$
\mathcal{Z}_{K} \simeq \bigvee_{k=3}^{\ell+1}\left(S^{k}\right)^{\wedge(k-2)\left(\begin{array}{c}
\ell \\
k-1
\end{array}\right)}
$$

where $\left(S^{k}\right)^{\wedge n}$ is the $n$-fold smash product of $S^{k}$ with itself. Comparing the formulas in (16) and (17) we see that the dimensions of the spheres correspond and the binomial coefficients match. This is not a coincidence. The order of the vertex cuts in producing the simple polytope $P$ does not influence the diffeomorphism type $[\mathrm{BM}]$, so one may choose a preferred order of the vertex cuts. The dual of a vertex cut polytope is a stacked polytope, which starts with $\Delta^{d}$ and iteratively glues on another copy of $\Delta^{d}$ along a common facet. In $[\mathrm{T}]$ a specific stacking order is chosen, producing a stacked polytope $\mathcal{L}$ of dimension $d$, satisfying the following.

Theorem 7.4. The stacked polytope $\mathcal{L}$ has the following properties:

(a) there is a homotopy equivalence $\mathcal{Z}_{\partial \mathcal{L}-\{1\}} \simeq \mathcal{Z}_{K_{\ell}}$ where $K_{\ell}$ is $\ell$ disjoint points;

(b) the inclusion $\partial \mathcal{L}-\{1\} \longrightarrow \partial \mathcal{L}$ induces a map $\mathcal{Z}_{\partial \mathcal{L}-\{1\}} \longrightarrow \mathcal{Z}_{\partial \mathcal{L}}$, which up to homotopy equivalences, is a map

$$
f: \bigvee_{k=3}^{\ell+1}\left(S^{k}\right)^{\wedge(k-2)\left(\begin{array}{c}
\ell \\
k-1
\end{array}\right)} \longrightarrow \#_{k=3}^{\ell+1}\left(S^{k} \times S^{\ell+2 d-k}\right)^{\#(k-2)\left(\begin{array}{c}
\ell \\
k-1
\end{array}\right)}
$$

(c) $f$ has a left homotopy inverse $g$;

(d) when restricted to a factor $H^{*}\left(S^{k} \times S^{\ell+2 d-k}\right)$ in the cohomology of the connected sum, $f^{*}$ is zero on precisely one of the ring generators.

Recently, Limonchenko [Li] showed that if $K=\partial P^{*}$ for $P$ a simple polytope and $K$ is minimally non-Golod then the simplicial multiwedge $K(J)$ is also minimally non-Golod for any sequence $J$. 
This is the combinatorial counterpart to Gitler-Lopez de Medrano's result on the diffeomorphism type of $\mathcal{Z}_{K(J)}$ being a connected sum of products of two spheres.

We close the survey by making some remarks on the subtlety of the connection between the statements:

(i) $K$ is Golod (that is, $\mathbf{k}[K]$ is Golod over every field $\mathbf{k}$ );

(ii) $\mathcal{Z}_{K}$ is homotopy equivalent to a wedge of spheres,

and

(iii) $K$ is minimally non-Golod;

(iv) $\mathcal{Z}_{K}$ is diffeomorphic to a connected sum of products of two spheres.

In [GPTW] it was shown that if $K$ is the minimal 6 -vertex triangulation of $\mathbb{R} P^{2}$ then $K$ is Golod but $\mathcal{Z}_{K}$ is not homotopy equivalent to a wedge of spheres. In fact, $\mathcal{Z}_{K}$ is homotopy equivalent to a wedge of spheres and a copy of $\Sigma^{7} \mathbb{R} P^{2}$. So a condition additional to Golodness is needed on the Stanley-Reisner face ring $\mathbb{Z}[K]$ to ensure that $\mathcal{Z}_{K}$ is homotopy equivalent to a wedge of spheres. However, even a torsion-free condition is not enough. Limonchenko [Li] showed that if $L$ is the minimal 9-vertex triangulation of $\mathbb{C} P^{2}$ then $L$ is Golod and has no torsion in the integral cohomology of any full subcomplex but $\mathcal{Z}_{K}$ is not homotopy equivalent to a wedge of spheres. In fact, $\mathcal{Z}_{L}$ is homotopy equivalent to a wedge of spheres and a copy of $\Sigma^{10} \mathbb{C} P^{2}$. Limonchenko goes on to show that this sort of problem persists in the minimally non-Golod case. If $L^{\prime}$ is $L$ with one stack along a maximal 4-simplex then $L^{\prime}$ is minimally non-Golod, has no torsion in the integral cohomology of any of its full subcomplexes, but $\mathcal{Z}_{L^{\prime}}$ is not homotopy equivalent to a connected sum of products of two spheres because of the existence of a non-trivial triple Massey product.

\section{Part 2. Higher Whitehead Products in Toric Topology}

In the second part of the paper we consider the map $\widetilde{w}$ in the homotopy fibration $\mathcal{Z}_{K} \stackrel{\widetilde{w}}{\longrightarrow} D J_{K} \longrightarrow$ $\prod_{i=1}^{m} \mathbb{C} P^{\infty}$ and identify it as a sum of higher and iterated Whitehead products for a certain family of simplicial complexes $K$.

\section{Statement of Results}

As seen in Part 1, there has been considerable success in studying the homotopy type of $\mathcal{Z}_{K}$ or its suspension. However, no attempt has been made to study the map $\mathcal{Z}_{K} \longrightarrow D J_{K}$. This means that the interesting information that is known about the moment-angle complex $\mathcal{Z}_{K}$ cannot be related to the Davis-Januszkiewicz space $D J_{K}$. The purpose of this paper is to remedy this deficiency. We show that for a certain family of simplicial complexes $K, \mathcal{Z}_{K}$ is homotopy equivalent to a wedge of spheres and the homotopy equivalence may be chosen so that the map $\mathcal{Z}_{K} \longrightarrow D J_{K}$ consists of a specified collection of higher Whitehead products and iterated Whitehead products. In particular, 
each missing face of $K$ corresponds to the existence of a non-trivial higher Whitehead product whose adjoint has a nonzero Hurewicz image in $H_{*}\left(\Omega D J_{K} ; \mathbb{Q}\right)$.

The focus in this part will be on $D J_{K}$ and $\mathcal{Z}_{K}$ but some of the results will be valid in a more general setting. To make the notation reflect this, we make the following definitions. Let $X_{1}, \ldots, X_{m}$ be path-connected pointed $C W$-complexes and let $\underline{X}=\left\{X_{1}, \ldots, X_{m}\right\}$. Let $K$ be a simplicial complex on the vertex set $[m]$. Define

$$
D J_{K}(\underline{X})=(\underline{X}, \underline{*})^{K} \quad \text { and } \quad \mathcal{Z}_{K}(\underline{X})=(\underline{C \Omega X}, \underline{\Omega X})^{K}
$$

By Theorem 5.5 there is a homotopy fibration

$$
\mathcal{Z}_{K}(\underline{X}) \longrightarrow D J_{K}(\underline{X}) \longrightarrow \prod_{i=1}^{m} X_{i}
$$

If $X_{1}, \ldots, X_{m}$ all equal a common space $X$, we instead write $\mathcal{Z}_{K}(X)$ and $D J_{K}(X)$. When $X_{i}=\mathbb{C} P^{\infty}$ for each $1 \leq i \leq m$, the homotopy fibration (18) specializes to the case of primary interest in toric topology, that is, to the homotopy fibration $\mathcal{Z}_{K} \longrightarrow D J_{K} \longrightarrow \prod_{i=1}^{m} \mathbb{C} P^{\infty}$.

The homology of $\Omega D J_{K}(\underline{X})$ with various coefficients has been calculated for different families of simplicial complexes. Some simple but important examples of the homology of $\Omega D J_{K}(\underline{X})$ were calculated by Lemaire [Le] in 1974 before the notion of $\mathcal{Z}_{K}(\underline{X})$ and $D J_{K}(\underline{X})$ were introduced. Panov and Ray $[\mathrm{PR}]$ introduced categorical formalism to study the homology of $\Omega D J_{K}(\underline{X})$ and gave explicit calculations when $K$ is a flag complex. Dobrinskaya [D] has a general approach for calculating the homology of $\Omega D J_{K}(\underline{X})$ for an arbitrary simplicial complex $K$ in terms of the homology of $\Omega(\underline{X})$ and some special relations coming from the homology of $\Omega D J_{K}\left(S^{2}\right)$. However, the homology of $\Omega \mathcal{Z}_{K}(\underline{X})$ remains a mystery.

In this paper we first consider the case when each $X_{i}$ is a sphere, writing $\underline{S}=\left(S^{n_{1}+1}, \ldots, S^{n_{m}+1}\right)$. As an intermediate goal towards understanding the map $\mathcal{Z}_{K} \longrightarrow D J_{K}$ we need to calculate the rational homology of $\Omega D J_{K}(\underline{S})$ and $\Omega D J_{K}$. However, it is important to emphasize that we do this in such a way as to remember the geometry of the space, that is, in such a way as to keep track of specific Hurewicz images. The existing models for rational loop homology are not known to do this, so we have to produce our own model which does. The methods we use lend themselves well to concrete calculations, and we include some examples to illustrate this.

In what follows $K$ will be a simplicial complex on the vertex set $[m]$. A simplex $\sigma \in K$ corresponds to a sequence $\left(i_{1}, \ldots, i_{k}\right)$ where $1 \leq i_{1}<\cdots<i_{k} \leq m$ and the integers $i_{j}$ are the vertices of $K$ which are in $\sigma$. Let $\operatorname{dim}(\sigma)=k-1$ be the dimension of $\sigma$. We concentrate on the collection $M F(K)$ of missing faces. To be precise, a sequence $\left(i_{1}, \ldots, i_{k}\right)$ is in $M F(K)$ if: (i) $\left(i_{1}, \ldots, i_{k}\right) \notin K$, and (ii) every proper subsequence of $\left(i_{1}, \ldots, i_{k}\right)$ is in $K$. For example, let $K$ be the simplicial complex on 4 
vertices

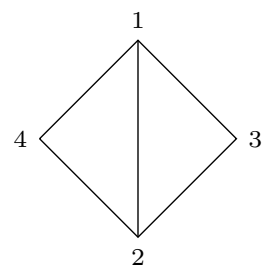

Then $M F(K)=\{(3,4),(1,2,3),(1,2,4)\}$.

Definition 8.1. Let $K$ be a simplicial complex the vertex set $[m]$. We say that $K$ is an $M F$-complex if

$$
|K|=\bigcup_{\sigma \in M F(K)}|\partial \sigma|
$$

where $|K|$ and $|\partial \sigma|$ denote the geometrical realisations of $K$ and $\partial \sigma$, respectively.

A simple example of a non- $M F$-complex is the boundary of a square.

The class of $M F$-complexes is larger than we want for producing wedge decompositions of $\mathcal{Z}_{K}(\underline{S})$ and $\mathcal{Z}_{K}$. We will give an example in Section 9 of an $M F$-complex $K$ with the property that $\mathcal{Z}_{K}$ has non-trivial cup-products. We wish to avoid this, so we add another condition which restricts how the faces of $K$ can be assembled.

Definition 8.2. Let $K$ be an $M F$-complex on the vertex set $[m]$. We say that $K$ is a directed $M F$-complex if there is a sequence of subcomplexes $\emptyset \subseteq K_{1} \subseteq \cdots \subseteq K_{l}=K$ for some $l$, where $K_{i}=K_{i-1} \cup \partial \sigma_{i}$ for $\sigma_{i} \in M F(K)$ and $K_{i-1} \cap \sigma_{i}$ is a face common to $K_{i-1}$ and $\sigma_{i}$.

Observe that the simplicial complex (19) is a directed $M F$-complex, but if the edge $(3,4)$ is also added (giving the 1-skeleton of a tetrahedron) then the resulting simplicial complex is an $M F$ complex but not a directed $M F$-complex. More examples are given in Section 9 .

Directed $M F$-complexes have the property that $\mathcal{Z}_{K}$ decomposes as a wedge of spheres. In particular, all cup products and higher Massey products in $H^{*}\left(\mathcal{Z}_{K}\right)$ are zero. We obtain this as a special case of a property for more general spaces.

Theorem 8.3. Let $K$ be a directed $M F$-complex on the vertex set $[m]$. Let $\underline{X}=\left\{X_{1}, \ldots, X_{m}\right\}$ where each $X_{i}$ is a path-connected topological space. Then $\mathcal{Z}_{K}(\underline{X})$ is homotopy equivalent to a wedge of spaces of the form $\Sigma^{t} \Omega X_{i_{1}} \wedge \cdots \wedge \Omega X_{i_{k}}$ for various $1 \leq t<m$ and sequences $\left(i_{1}, \ldots, i_{k}\right)$ where $1 \leq i_{1}<\cdots<i_{k} \leq m$.

Corollary 8.4. Let $K$ be a directed $M F$-complex on the vertex set $[m]$. Then each of $\mathcal{Z}_{K}(\underline{S})$ and $\mathcal{Z}_{K}$ is homotopy equivalent to a wedge of simply-connected spheres. 
Next, as an intermediate step, we calculate $H_{*}\left(\Omega D J_{K}(\underline{S}) ; \mathbb{Q}\right)$ and $H_{*}\left(\Omega D J_{K} ; \mathbb{Q}\right)$ using an AdamsHilton model, with the emphasis on detecting Hurewicz images. To state this we introduce some notation. If $V$ is a graded $\mathbb{Q}$-vector space, let $L\langle V\rangle$ be the free graded Lie algebra generated by $V$, and let $U L\langle V\rangle$ be its universal enveloping algebra. If $V$ has basis $\left\{v_{1}, \ldots, v_{m}\right\}$ let $L_{d s}\left\langle v_{1}, \ldots, v_{m}\right\rangle$ be the direct sum $\oplus_{i=1}^{m} L\left\langle v_{i}\right\rangle$. In particular, in $L_{d s}\left\langle v_{1}, \ldots, v_{m}\right\rangle$ we have $\left[v_{i}, v_{j}\right]=0$ if $i \neq j$. Notice that if $v_{i}$ is of even degree then $\left[v_{i}, v_{i}\right]=0$ but this is not the case if $v_{i}$ is of odd degree. On the other hand, if $v_{i}$ is of odd degree then in $U L\left\langle v_{i}\right\rangle$ we have $\left[v_{i}, v_{i}\right]=2 v_{i}^{2}$, so for $v_{i}$ of any parity we have $U L\left\langle v_{i}\right\rangle \cong \mathbb{Q}\left[v_{i}\right]$. Thus $U L_{d s}\left\langle v_{1}, \ldots, v_{m}\right\rangle \cong \otimes_{i=1}^{m} U L\left\langle v_{i}\right\rangle$. If $L$ is a Lie algebra and $x_{1}, \ldots, x_{k} \in L$, let $\left[\left[x_{1}, x_{2}\right], \ldots, x_{k}\right]$ denote the iterated bracket $\left[\ldots\left[\left[x_{1}, x_{2}\right], x_{3}\right], \ldots, x_{k}\right]$.

Let $b_{i}$ be the Hurewicz image of the adjoint of the coordinate inclusion $S^{n_{i}+1} \longrightarrow D J_{K}(\underline{S})$. Abusing notation, let $b_{i}$ also be the Hurewicz image of the adjoint of the composite $S^{2} \longrightarrow \mathbb{C} P^{\infty} \longrightarrow$ $D J_{K}$, where the left map is the inclusion of the bottom cell and the right map is the inclusion of the $i^{t h}$-coordinate. By $u_{\sigma}$ we denote the Hurewicz image of the adjoint of the Whitehead product corresponding to a missing face $\sigma \in M F(K)$. We will phrase $H_{*}\left(\Omega D J_{K}(\underline{S}) ; \mathbb{Q}\right)$ and $H_{*}\left(\Omega D J_{K} ; \mathbb{Q}\right)$ as quotients of $U\left(L_{d s}\left\langle b_{1}, \ldots, b_{m}\right\rangle \coprod L\left\langle u_{\sigma} \mid \sigma \in M F(K)\right\rangle\right)$. A distinction needs to be made between the elements $u_{\sigma}$ where $|\sigma|=2$ and $|\sigma|>2$. The latter elements are independent from $b_{1}, \ldots, b_{m}$. On the other hand, if $|\sigma|=2$ then $\sigma=\left(i_{1}, i_{2}\right)$ and $u_{\sigma}=\left[b_{i_{1}}, b_{i_{2}}\right]$, which is not independent from $b_{1}, \ldots, b_{m}$. This leads to additional relations determined by the graded Jacobi identity and face relations. Specifically, we have $\left[u_{\sigma}, b_{j}\right]=\left[\left[b_{i_{1}}, b_{i_{2}}\right], b_{j}\right]=\left[b_{i_{1}},\left[b_{i_{2}}, b_{j}\right]\right]-(-1)^{\left|b_{i_{1}}\right|\left|b_{i_{2}}\right|}\left[b_{i_{2}},\left[b_{i_{1}}, b_{j}\right]\right]$ and if $\left(i_{1}, j\right) \in K$ or $\left(i_{2}, j\right) \in K$ then $\left[b_{i_{1}}, b_{j}\right]=0$ or $\left[b_{i_{2}}, b_{j}\right]=0$ repsectively. The collection of such relations forms an ideal in $U\left(L_{d s}\left\langle b_{1}, \ldots, b_{m}\right\rangle \coprod L\left\langle u_{\sigma} \mid \sigma \in M F(K)\right\rangle\right)$ which we label as $J$. Note that if every missing face $\sigma \in M F(K)$ is of dimension greater than 1 , then $J$ is trivial.

Theorem 8.5. Let $K$ be a directed $M F$-complex. There is an algebra isomorphism

$$
H_{*}\left(\Omega D J_{K}(\underline{S}) ; \mathbb{Q}\right) \cong U\left(L_{d s}\left\langle b_{1}, \ldots, b_{m}\right\rangle \amalg L\left\langle u_{\sigma} \mid \sigma \in M F(K)\right\rangle\right) / J
$$

where each $u_{\sigma}$ is the Hurewicz image of the adjoint of a higher Whitehead product. Further, the loop map $\Omega D J_{K}(\underline{S}) \longrightarrow \prod_{i=1}^{m} \Omega S^{n_{i}+1}$ is modelled by the map

$$
U\left(L_{d s}\left\langle b_{1}, \ldots, b_{m}\right\rangle \amalg L\left\langle u_{\sigma} \mid \sigma \in M F(K)\right\rangle\right) / J \stackrel{U(\pi)}{\longrightarrow} U L_{d s}\left\langle b_{1}, \ldots, b_{m}\right\rangle
$$

where $\pi$ is the projection.

Let $\imath: S^{2} \longrightarrow \mathbb{C} P^{\infty}$ be the inclusion of the bottom cell. For any simplicial complex $K$, this induces a map $D J_{k}(\iota): D J_{K}\left(S^{2}\right) \longrightarrow D J_{K}$.

Theorem 8.6. Let $K$ be a directed $M F$-complex. There is an algebra isomorphism

$$
H_{*}\left(\Omega D J_{K} ; \mathbb{Q}\right) \cong U\left(L_{d s}\left\langle b_{1}, \ldots, b_{m}\right\rangle \amalg L\left\langle u_{\sigma} \mid \sigma \in M F(K)\right\rangle\right) /(I+J)
$$


where $u_{\sigma}$ is the Hurewicz image of the adjoint of a higher Whitehead product and I is the ideal

$$
I=\left(b_{i}^{2},\left[u_{\sigma}, b_{j_{\sigma}}\right] \mid 1 \leq i \leq m, \sigma=\left(i_{1}, \ldots, i_{k}\right) \in M F(K), j_{\sigma} \in\left\{i_{1}, \ldots, i_{k}\right\}\right) .
$$

Further, there is a commutative diagram of algebras

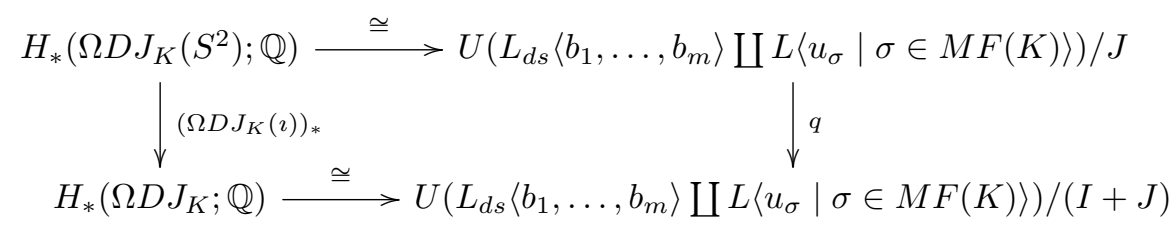

where $q$ is the quotient map.

Notice that the relation $b_{i}^{2}$ in $I$ lets us replace $L_{d s}\left\langle b_{1}, \ldots, b_{m}\right\rangle$ in the statement of Theorem 8.6 by $L_{a b}\left\langle b_{1}, \ldots, b_{m}\right\rangle$, where $L_{a b}$ is the free abelian Lie algebra generated by the indicated elements, which is characterized by having its bracket identically zero.

Our main theorems are homotopy theoretic. For $1 \leq i \leq m$, let $a_{i}: S^{n_{i}+1} \longrightarrow D J_{K}(\underline{S})$ be the inclusion of the $i^{t h}$-coordinate. The analogue of the algebraic ideal $J$ occurs when $\sigma=\left(i_{1}, i_{2}\right)$, in which case there is a Whitehead product $w_{\sigma}=\left[a_{i_{1}}, a_{i_{2}}\right]$; as in the algebraic case, this imposes relations determined by the Jacobi identity and face relations in cases of the form $\left[w_{\sigma}, a_{j}\right]$ when $\left(i_{1}, j\right) \in K$ or $\left(i_{2}, j\right) \in K$. For $|\sigma|=2$, let $W_{\sigma}$ be the collection of all independent Whitehead products of the form $\left[\left[w_{\sigma}, a_{j_{1}}\right] \ldots, a_{j_{l}}\right]$, where $1 \leq j_{1} \leq \cdots \leq j_{l} \leq l$ and $1 \leq l<\infty$.

Theorem 8.7. Let $K$ be a directed $M F$-complex on the vertex set $[m]$, so that there is a homotopy equivalence $\mathcal{Z}_{K}(\underline{S}) \simeq \bigvee_{\alpha \in \mathcal{I}} S^{t_{\alpha}}$. The equivalence can be chosen so that the composite

$$
\bigvee_{\alpha \in \mathcal{I}} S^{t_{\alpha}} \longrightarrow \mathcal{Z}_{K}(\underline{S}) \longrightarrow D J_{K}(\underline{S})
$$

is a wedge sum of the following maps:

(a) a higher Whitehead product $w_{\sigma}: S^{t_{\sigma}} \longrightarrow D J_{K}(\underline{S})$ for each missing face $\sigma=$ $\left(i_{1}, \ldots, i_{k}\right) \in M F(K)$, where $t_{\sigma}=k-1+\left(\sum_{j=1}^{k} n_{i_{j}}\right) ;$

(b) an iterated Whitehead product

$$
\left[\left[w_{\sigma}, a_{j_{1}}\right] \ldots, a_{j_{l}}\right]: S^{t_{\alpha}} \longrightarrow D J_{K}(\underline{S})
$$

for each $\sigma \in M F(K)$ of dimension greater than 1 and each list $1 \leq j_{1} \leq \cdots \leq j_{l} \leq l$, where $1 \leq l<\infty$ and $t_{\alpha}=t_{\sigma}+\sum_{t=1}^{l} n_{j_{t}}$;

(c) the collection of independent iterated Whitehead products $W_{\sigma}$ for each $\sigma \in M F(K)$ of dimension 1.

Let $\tilde{a}_{i}$ be the composite $\tilde{a}_{i}: S^{2} \stackrel{\imath}{\longrightarrow} \mathbb{C} P^{\infty} \longrightarrow D J_{K}$ where the right map is the inclusion of the $i^{t h}$-coordinate. If $\sigma=\left(i_{1}, i_{2}\right)$, let $\widetilde{w}_{\sigma}$ be the Whitehead product $\left[\tilde{a}_{i_{1}}, \tilde{a}_{i_{2}}\right]$. As above, for $|\sigma|=2$, let $\widetilde{W}_{\sigma}$ be the collection of all independent Whitehead products of the form $\left[\left[\widetilde{w}_{\sigma}, \tilde{a}_{j_{1}}\right] \ldots, \tilde{a}_{j_{l}}\right]$ where $1 \leq j_{1} \leq \cdots \leq j_{l} \leq l$ and $1 \leq l<\infty$. Given $\sigma=\left(i_{1}, \ldots, i_{k}\right)$, let $J_{\sigma}=\{1, \ldots, n\}-\left\{i_{1}, \ldots, i_{k}\right\}$. 
Theorem 8.8. Let $K$ be a directed $M F$-complex on the vertex set $[m]$, so that there is a homotopy equivalence $\mathcal{Z}_{K} \simeq \bigvee_{\widetilde{\alpha} \in \tilde{\mathcal{I}}} S^{t \tilde{\alpha}}$. The equivalence can be chosen so that the composite

$$
\bigvee_{\widetilde{\alpha} \in \widetilde{\mathcal{I}}} S^{t \tilde{\alpha}} \longrightarrow \mathcal{Z}_{K} \longrightarrow D J_{K}
$$

is a wedge sum of the following maps:

(a) a higher Whitehead product $\widetilde{w}_{\sigma}: S^{2|\sigma|-1} \longrightarrow D J_{K}$ for each missing face $\sigma \in$ $M F(K)$;

(b) an iterated Whitehead product

$$
\left[\left[\widetilde{w}_{\sigma}, \tilde{a}_{j_{1}}\right] \ldots, \tilde{a}_{j_{l}}\right]: S^{2|\sigma|+l-1} \longrightarrow D J_{K}
$$

for each $\sigma \in M F(K)$ of dimension greater than 1 and each list $j_{1}<\cdots<j_{l}$ in $J_{\sigma}$, where $1 \leq l \leq m$;

(c) the collection of independent iterated Whitehead products $\widetilde{W}_{\sigma}$ for each $\sigma \in M F(K)$ of dimension 1.

Although in this paper our goal is to identify the map $\mathcal{Z}_{K} \longrightarrow D J_{K}$ for $K$ a directed $M F$ complex, we expect this can be generalized first to a much larger family of simplicial complexes and second to the map between polyhedral products $\mathcal{Z}_{K}(\underline{X}) \longrightarrow D J_{K}(\underline{X})$.

\section{The obJECTS OF STUdY}

This section gives an initial analysis of directed $M F$-complexes. First, we compare directed $M F$ complexes to another family of simplicial complexes that has received considerable attention for its role in producing wedge decompositions of $\mathcal{Z}_{K}$. Then we prove the wedge decompositions in Theorem 8.3 and Corollary 8.4.

Recall that a simplicial complex $K$ on $n$ vertices is shifted if there is an ordering on the vertex set such that whenever $\sigma$ is a simplex of $K$ and $v^{\prime}<v$, then $(\sigma-v) \cup v^{\prime}$ is a simplex of $K$. In [GT2] it was shown that if $K$ is a shifted complex then $\mathcal{Z}_{K}$ is homotopy equivalent to a wedge of spheres. In fact, in [GT2] it was shown that if $K$ is shifted then the polyhedral product $(\underline{C \Omega X}, \underline{\Omega X})$ is homotopy equivalent to a wedge of suspensions. This was generalised to any polyhedral product $(\underline{C X}, \underline{X})^{K}$ in [GT3, IK1]. 
We show that directed $M F$-complexes and shifted complexes form distinct families, with nontrivial intersection. Consider the three examples:

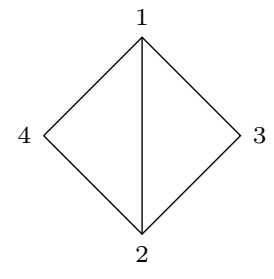

$K_{1}$

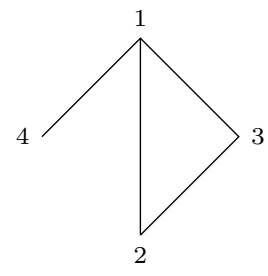

$K_{2}$

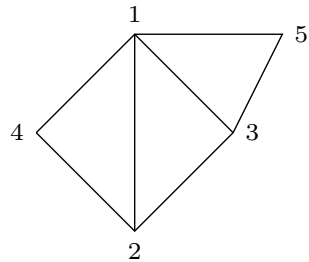

$K_{3}$

Observe that $K_{1}$ and $K_{2}$ are shifted but $K_{3}$ is not. The list of minimal missing faces in each case is:

$$
\begin{aligned}
& M F\left(K_{1}\right)=\{(3,4),(1,2,3),(1,2,4)\} \\
& M F\left(K_{2}\right)=\{(2,4),(3,4),(1,2,3)\} \\
& M F\left(K_{3}\right)=\{(2,5),(3,4),(4,5),(1,2,3),(1,2,4),(1,3,5)\} .
\end{aligned}
$$

Observe that $\left|K_{1}\right|=\bigcup_{\sigma \in M F\left(K_{1}\right)}|\partial \sigma|$ and $\left|K_{3}\right|=\bigcup_{\sigma \in M F\left(K_{3}\right)}|\partial \sigma|$, but in contrast, $\bigcup_{\sigma \in M F\left(K_{2}\right)}|\partial \sigma|=$ $\left|K_{2}-(1,4)\right|$. As well, $K_{1}$ can be formed by gluing the boundary of $(1,2,3)$ to the boundary of $(1,2,4)$ along the common face $(1,2)$, and $K_{3}$ can be formed by gluing the boundary of $(1,3,5)$ to $K_{1}$ along the common face $(1,3)$. Thus $K_{1}$ is a shifted complex which is also a directed $M F$-complex, while $K_{2}$ is a shifted complex which is not a directed $M F$-complex, and $K_{3}$ is a directed $M F$-complex which is not shifted.

As noted in Section 8, a simple example of an $M F$-complex which is not a directed $M F$-complex is the 1-skeleton of a tetrahedron. However, by [GT2], in this case the conclusions of Theorem 8.3 and Corollary 8.4 nevertheless hold; in particular, $\mathcal{Z}_{K}$ is homotopy equivalent to a wedge of spheres. It is useful to also give an example of an $M F$-complex which is not a directed $M F$-complex and for which Theorem 8.3 and Corollary 8.4 fail. Let $K$ be the simplicial complex on 8 vertices given by

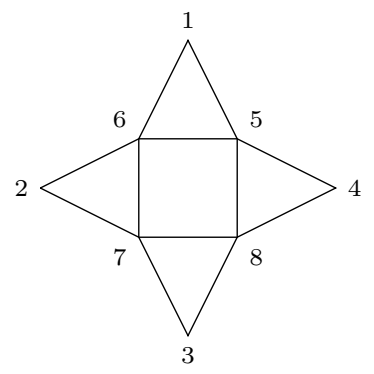

Notice that $K$ is the union of the boundaries of the faces $\{(1,5,6),(2,6,7),(3,7,8),(4,5,8)\}$. This implies that $|K|=\bigcup_{\sigma \in M F(K)}|\partial \sigma|$, so $K$ is an $M F$-complex. However, $K$ is not a directed $M F$ complex. For, thinking of $|K|$ as the union of the boundaries of four triangles, it is possible to glue the second triangle to the first and the third to the first two along a common vertex, but the fourth triangle is glued to the first three along two vertices, which is not a common face. In this case the 
existence of the boundary of the square in $K$ leads to non-trivial cup products in the cohomology of $\mathcal{Z}_{K}$, implying that $\mathcal{Z}_{K}$ cannot be a wedge of spheres.

Now we turn to the homotopy types of $\mathcal{Z}_{K}(\underline{X})$ and $\mathcal{Z}_{K}$ when $K$ is a directed $M F$-complex. For clarity, we restate Theorem 8.3 and Corollary 8.4.

Theorem 9.1. Let $K$ be a directed $M F$-complex on the vertex set $[m]$. Let $\underline{X}=\left\{X_{1}, \ldots, X_{m}\right\}$ where each $X_{i}$ is a path-connected, pointed $C W$-complex. Then $\mathcal{Z}_{K}(\underline{X})$ is homotopy equivalent to a wedge of spaces of the form $\Sigma^{t} \Omega X_{i_{1}} \wedge \cdots \wedge \Omega X_{i_{k}}$ for various $1 \leq t<m$ and sequences $\left(i_{1}, \ldots, i_{k}\right)$ where $1 \leq i_{1}<\cdots<i_{k} \leq m$.

Proof. Write $Y \in \mathcal{W}$ if $Y$ is a space that is homotopy equivalent to a wedge of spaces of the form $\Sigma^{t} \Omega X_{i_{1}} \wedge \cdots \wedge \Omega X_{i_{k}}$ for various $1 \leq t<m$ and sequences $\left(i_{1}, \ldots, i_{k}\right)$ where $1 \leq i_{1}<\cdots<i_{k} \leq m$. For any such sequence, let $F W\left(i_{1}, \ldots, i_{k}\right)$ be the fat wedge of the product $\prod_{j=1}^{k} X_{i_{j}}$. By definition, the homotopy fibre of the inclusion $F W\left(i_{1}, \ldots, i_{k}\right) \longrightarrow \prod_{j=1}^{k} X_{i_{j}}$ is $\mathcal{Z}_{K}$ for $K=\left(\Delta^{k}\right)_{k-1}=\partial \sigma$. On the other hand, by [Po2], this homotopy fibre is homotopy equivalent to $\Sigma^{k-1} \Omega X_{i_{1}} \wedge \cdots \wedge \Omega X_{i_{k}}$. In particular, in an $M F$-complex $|K|=\bigcup_{\sigma \in M F(K)}|\partial \sigma|$, we have $\mathcal{Z}_{\partial \sigma} \in \mathcal{W}$ for every $\sigma \in M F(K)$.

By definition, since $K$ is a directed $M F$-complex, there is a sequence of subcomplexes $\emptyset \subseteq K_{1} \subseteq$ $\cdots \subseteq K_{l}=K$ for some $l$, where $K_{i}=K_{i-1} \cup \partial \sigma_{i}$ for $\sigma_{i} \in M F(K)$ and $K_{i-1} \cap \sigma_{i}$ is a face common to $K_{i-1}$ and $\sigma_{i}$. We proceed with the proof by induction. Since $K_{1}=\partial \sigma_{1}$, the previous paragraph shows that $\mathcal{Z}_{K_{1}} \in \mathcal{W}$. Now suppose that $\mathcal{Z}_{K_{i-1}} \in \mathcal{W}$. We have $K_{i}$ constructed by gluing $\partial \sigma_{i}$ and $K_{i-1}$ along a common face, $\mathcal{Z}_{\partial \sigma_{i}} \in \mathcal{W}$ by the preceding paragraph, and $\mathcal{Z}_{K_{i-1}} \in \mathcal{W}$ by assumption. Under these circumstances, [GT2, Theorem 1.3] implies that $Z_{K_{i}} \in \mathcal{W}$. (Actually, [GT2, Theorem 1.3] is stated for the special case when $X_{i}=\mathbb{C} P^{\infty}$ for $1 \leq i \leq n$, but the proof goes through without change in the general case.) Hence, by induction, $\mathcal{Z}_{K}(\underline{X})=\mathcal{Z}_{K_{l}}$ is in $\mathcal{W}$.

Corollary 9.2. Let $K$ be a directed $M F$-complex on the vertex set $[m]$. Then $\mathcal{Z}_{K}(\underline{S})$ is homotopy equivalent to an infinite wedge of simply-connected spheres, and $\mathcal{Z}_{K}$ is homotopy equivalent to a finite wedge of simply-connected spheres.

Proof. In Theorem 8.3, suppose that $X_{i}=S^{n_{i}+1}$ for each $1 \leq i \leq m$. Then $\mathcal{Z}_{K}(\underline{S})$ is homotopy equivalent to a wedge of spaces of the form $\Sigma^{t} \Omega S^{n_{i_{1}}+1} \wedge \cdots \wedge \Omega S^{n_{i_{k}}+1}$ for various $1 \leq t<m$ and sequences $\left(i_{1}, \ldots, i_{k}\right)$. By $[\mathrm{J}]$, there is a homotopy equivalence $\Sigma \Omega S^{n_{i}+1} \simeq \bigvee_{j=1}^{\infty} S^{j n_{i}+1}$. Since $S^{j n_{i}+1}$ is a suspension, iterating this homotopy equivalence shows that each wedge summand $\Sigma^{t} \Omega S^{n_{i_{1}}+1} \wedge \cdots \wedge \Omega S^{n_{i_{k}}+1}$ is homotopy equivalent to an infinite wedge of simply-connected spheres. Thus $\mathcal{Z}_{K}(\underline{S})$ is homotopy equivalent to an infinite wedge of simply-connected spheres.

Next, in Theorem 8.3, suppose that $X_{i}=\mathbb{C} P^{\infty}$ for each $1 \leq i \leq m$. Then $\mathcal{Z}_{K}$ is homotopy equivalent to a wedge of spaces of the form $\Sigma^{t} \Omega \mathbb{C} P_{i_{1}}^{\infty} \wedge \cdots \wedge \Omega \mathbb{C} P_{i_{k}}^{\infty}$ for various $1 \leq t<m$ and sequences $\left(i_{1}, \ldots, i_{k}\right)$. Since $\Omega \mathbb{C} P^{\infty} \simeq S^{1}$, each wedge summand $\Sigma^{t} \Omega \mathbb{C} P_{i_{1}}^{\infty} \wedge \cdots \wedge \Omega \mathbb{C} P_{i_{k}}^{\infty}$ is homotopy 
equivalent to $S^{k+t}$. Thus $\mathcal{Z}_{K}$ is homotopy equivalent to a finite wedge of simply-connected spheres.

\section{Higher Whitehead Products and Fat Wedges}

In this section we define a higher Whitehead product by means of a fat wedge, and relate the existence of a missing face in $K$ to the existence of a non-trivial higher Whitehead in $D J_{K}(\underline{X})$. Let $X_{1}, \ldots, X_{m}$ be path-connected spaces and let $\underline{X}=\left\{X_{1}, \ldots, X_{m}\right\}$. The fat wedge is the space

$$
F W(\underline{X})=\left\{\left(x_{1}, \ldots, x_{m}\right) \in X_{1} \times \cdots \times X_{m} \mid \text { at least one } x_{i}=*\right\} .
$$

Consider the homotopy fibration obtained by including $F W(\underline{X})$ into the product $X_{1} \times \cdots \times X_{m}$. The homotopy type of the fibre was first identified by Porter [Po2], who showed that there is a homotopy fibration

$$
\Sigma^{m-1} \Omega X_{1} \wedge \cdots \wedge \Omega X_{m} \longrightarrow F W(\underline{X}) \longrightarrow X_{1} \times \cdots \times X_{m}
$$

There is a reformulation of this in terms of the polyhedral product. Let $K=\partial \Delta^{m-1}$, the boundary of the $(m-1)$-simplex. Let $C Y$ be the reduced cone on $Y$, parameterised as $C Y=[0,1] \times Y / \sim$ for $(0, y) \sim *$ and $(t, *) \sim *$. Note that the cone point is at 0 . Observe that there is a map of pairs $\left(C \Omega X_{i}, \Omega X_{i}\right) \longrightarrow\left(X_{i}, *\right)$ given by sending $(s, \omega) \in C \Omega X_{i}$ to $\omega(s)$. Then, essentially by [Po2], the map $\Sigma^{n-1} \Omega X_{1} \wedge \cdots \wedge \Omega X_{m} \longrightarrow F W(\underline{X})$ can be identified with the map $(\underline{C \Omega X}, \underline{\Omega X})^{K} \longrightarrow(\underline{X}, \underline{*})^{K}$.

If each $X_{i}$ is a suspension, $X_{i}=\Sigma Y_{i}$, then the suspension map $E: Y \longrightarrow \Omega \Sigma Y$ induces a composite

$$
\phi_{m}: \Sigma^{m-1} Y_{1} \wedge \cdots \wedge Y_{m} \longrightarrow \Sigma^{m-1} \Omega \Sigma Y_{1} \wedge \cdots \wedge \Omega \Sigma Y_{m} \longrightarrow F W(\underline{\Sigma Y}) .
$$

The map $\phi_{m}$ is the attaching map that yields the product. That is, there is a homotopy cofibration

$$
\Sigma^{m-1} Y_{1} \wedge \cdots \wedge Y_{m} \stackrel{\phi_{m}}{\longrightarrow} F W(\underline{\Sigma Y}) \longrightarrow \Sigma Y_{1} \times \cdots \times \Sigma Y_{m}
$$

In the case $m=2$, we have $F W(\underline{\Sigma Y})=\Sigma Y_{1} \vee \Sigma Y_{2}$ and $\phi_{2}$ is the Whitehead product $\left[i_{1}, i_{2}\right]$, where $i_{1}$ and $i_{2}$ are the inclusions of $\Sigma Y_{1}$ and $\Sigma Y_{2}$ respectively into $\Sigma Y_{1} \vee \Sigma Y_{2}$. This is the universal example for Whitehead products. Given a space $Z$ and maps $f: \Sigma Y_{1} \longrightarrow Z$ and $g: \Sigma Y_{2} \longrightarrow Z$, the Whitehead product $[f, g]$ of $f$ and $g$ is the composite $\Sigma Y_{1} \wedge Y_{2} \stackrel{\phi_{2}}{\longrightarrow} \Sigma Y_{1} \vee \Sigma Y_{2} \stackrel{f \perp g}{\longrightarrow} Z$, where $\perp$ denotes the wedge sum. Porter [Po1] used the maps $\phi_{m}$ for $m>2$ as universal examples to define higher Whitehead products.

Definition 10.1. For $m \geq 2$, let $Y_{1}, \ldots, Y_{m}$ and $Z$ be path-connected spaces, and let $f_{i}: \Sigma Y_{i} \longrightarrow$ $Z$ be maps. Suppose that the wedge sum $\bigvee_{i=1}^{m} \Sigma Y_{i} \longrightarrow Z$ of the maps $f_{i}$ extends to a map $f: F W(\underline{\Sigma Y}) \longrightarrow Z$. The $m^{\text {th }}$-higher Whitehead product of the maps $f_{1}, \ldots, f_{m}$ is the composite

$$
\left[f_{1}, \ldots, f_{m}\right]: \Sigma^{m-1} Y_{1} \wedge \cdots \wedge Y_{m} \stackrel{\phi_{m}}{\longrightarrow} F W(\underline{\Sigma Y}) \stackrel{f}{\longrightarrow} Z .
$$


If $m=2$, the Whitehead product of two maps $f_{1}$ and $f_{2}$ is always defined, and the homotopy class of $\left[f_{1}, f_{2}\right]$ is uniquely determined by the homotopy classes of $f_{1}$ and $f_{2}$. If $m>2$, it may not be the case that the higher Whitehead product of $m$ maps $f_{1}, \ldots, f_{m}$ exists, as there may be non-trivial obstructions to extending the given map $\bigvee_{i=1}^{m} \Sigma Y_{i} \longrightarrow Z$ to the fat wedge $F W(\underline{\Sigma Y})$. Even if such an extension exists, there may be many inequivalent choices of an extension, implying that the homotopy class of $\left[f_{1}, \ldots, f_{m}\right]$ need not be uniquely determined by the homotopy classes of $f_{1}, \ldots, f_{m}$.

When $m=2$, the adjoint of the Whitehead product $\left[f_{1}, f_{2}\right]$ is homotopic to a Samelson product. Its image in homology is given by commutators. We wish to have analogous information about higher Whitehead products. The universal example is given by the adjoint of $\phi_{m}$, which is a map $\Sigma^{m-2} Y_{1} \wedge \cdots \wedge Y_{m} \longrightarrow \Omega F W(\underline{\Sigma Y})$. We want to know the Hurewicz image of this map. To do so we need a good model for $H_{*}(\Omega F W(\underline{\underline{Y}}))$ which sees this Hurewicz image. Producing such a model in the case when each $Y_{i}$ is a sphere is the subject of Section 11.

Before getting to this, we give a general result which identifies non-trivial higher Whitehead products in $D J_{K}(\underline{\underline{\Sigma}})$ for any simplicial complex $K$. In short, there is a non-trivial higher Whitehead product for each missing face of $K$ which, moreover, lifts to $\mathcal{Z}_{K}(\underline{\Sigma Y})$. In what follows we will consider sub-products $\prod_{j=1}^{k} \Sigma Y_{i_{j}}$ of $\prod_{i=1}^{m} \Sigma Y$. If $\sigma=\left(i_{1}, \ldots, i_{k}\right)$, let $F W(\underline{\Sigma Y}, \sigma)$ be the fat wedge of the sub-product $\prod_{j=1}^{k} \Sigma Y_{i_{j}}$.

Lemma 10.2. Let $K$ be a simplicial complex on the vertex set $[m]$. If $\sigma=\left(i_{1}, \ldots, i_{k}\right) \in M F(K)$ then there exists maps $f_{\sigma}: F W(\underline{\Sigma Y}, \sigma) \longrightarrow D J_{K}(\underline{\Sigma Y})$ and $g_{\sigma}: \Sigma^{k-1} Y_{i_{1}} \wedge \cdots \wedge Y_{i_{k}} \longrightarrow \mathcal{Z}_{K}(\underline{\Sigma Y})$ which both have left homotopy inverses, and which fit into a homotopy commutative diagram

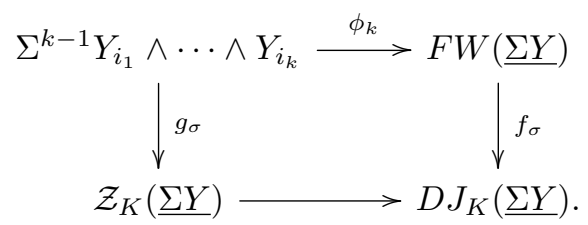

Proof. Since $\sigma \in M F(K)$, it is the full subcomplex of $K$ on the vertex set $\left\{i_{1}, \ldots, i_{k}\right\}$. Therefore, if $(\underline{X}, \underline{A})$ is any $n$ pairs of $C W$-complexes $\left(X_{i}, A_{i}\right)$ then the polyhedral product $(\underline{X}, \underline{A})^{\sigma}$ is a natural retract of $(\underline{X}, \underline{A})^{K}$. Applying this to the map $(\underline{C \Omega \Sigma Y}, \underline{\Omega \Sigma Y}) \longrightarrow(\underline{\Sigma Y}, \underline{*})$ obtained from the maps of pairs $\left(C \Omega \Sigma Y_{i}, \Omega \Sigma Y_{i}\right) \longrightarrow\left(\Sigma Y_{i}, *\right)$, we obtain a homotopy commutative diagram

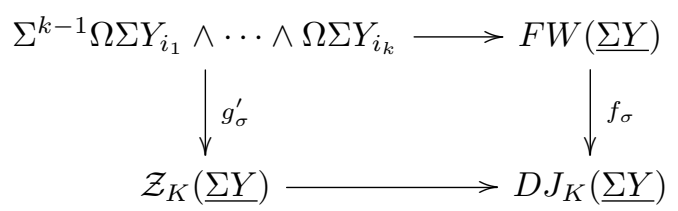

where $f_{\sigma}$ and $g_{\sigma}^{\prime}$ have left homotopy inverses. Precompose the diagram with the map

$$
\epsilon: \Sigma^{k-1} Y_{i_{1}} \wedge \cdots \wedge Y_{i_{k}} \longrightarrow \Sigma^{k-1} \Omega \Sigma Y_{i_{1}} \wedge \cdots \wedge \Omega \Sigma Y_{i_{k}}
$$


induced by the suspension maps $Y_{i} \stackrel{E}{\longrightarrow} \Omega \Sigma Y_{i}$. By definition, $\phi_{k}$ is $\epsilon$ composed with the upper horizontal map in the preceding diagram, so if let $g_{\sigma}=g_{\sigma}^{\prime} \circ \epsilon$ then we obtain the homotopy commutative diagram asserted by the lemma.

It remains to show that $g_{\sigma}$ has a left homotopy inverse. But as $\Sigma E$ has a left homotopy inverse, so does $\epsilon$, and therefore as $g_{\sigma}^{\prime}$ has a left homotopy inverse, so does $g_{\sigma}$.

\section{Adams-Hilton models}

Let $X$ be a simply-connected $C W$-complex of finite type and $R$ a commutative ring. The AdamsHilton model $[\mathrm{AH}]$ is a means of calculating $H_{*}(\Omega X ; R)$. One advantage it has over other models for $H_{*}(\Omega X ; R)$ is its relative simplicity, which allows for concrete calculations in certain cases. Its presentation is stated in Theorem 11.1.

Let $V$ be a graded $R$-module, and let $T(V)$ be the free tensor algebra on $V$. For a space $X$, let $C U_{*}(X)$ be the cubical singular chain complex on $X$ with coefficients in $R$. Note that $C U_{*}(X)$ is naturally chain equivalent to the simplicial singular chain complex on $X$. If $X$ is a homotopy associative $H$-space then the multiplication on $X$ induces a multiplication on $C U_{*}(X)$, giving it the structure of a differential graded algebra. A map $A \longrightarrow B$ of differential graded algebras is a quasi-isomorphism if it induces an isomorphism in homology.

Theorem 11.1. Let $R$ be a commutative ring and let $X$ be a simply-connected $C W$-complex of finite type. The Adams-Hilton model for $X$ is a differential graded $R$-algebra $A H(X)$ satisfying:

(a) if $X=p t \cup\left(\bigcup_{\alpha \in S} e_{\alpha}\right)$ is a $C W$-decomposition of $X$ then $A H(X)=T\left(V ; d_{V}\right)$ where $V=\left\{b_{\alpha}\right\}_{\alpha \in S}$ and $\left|b_{\alpha}\right|=\left|e_{\alpha}\right|-1$;

(b) the differential $d_{V}$ depends on the attaching maps of the $C W$-complex $X$;

(c) there is a map of differential graded algebras $\theta_{X}: A H(X) \longrightarrow C U_{*}(\Omega X)$ which induces an isomorphism $H_{*}(A H(X)) \cong H_{*}(\Omega X ; R)$.

Notice that the generators of $A H(X)$ are in one-to-one correspondence with the cells of $X$, shifted down by one dimension. However, the differential $d_{V}$ and the quasi-isomorphism $\theta_{X}$ are not uniquely determined by the $C W$-structure of $X$. There may be many inequivalent choices of both $d_{V}$ and $\theta_{X}$ which result in an isomorphism $H_{*}(A H(X)) \cong H_{*}(\Omega X ; R)$. In that sense, there may be many Adams-Hilton models for $H_{*}(\Omega X ; R)$. One would hope to choose a model which is particularly advantageous. This is what we aim to do for $X=D J_{K}(\underline{S})$ or $D J_{K}$ and $R=\mathbb{Q}$ by choosing a model which keeps track of the Hurewicz images of adjointed higher Whitehead products.

We start with some general constructions in the case of $D J_{K}\left(S^{2}\right)$ and $D J_{K}$, producing AdamsHilton models for both which are compatible with the inclusion $S^{2} \stackrel{\imath}{\longrightarrow} \mathbb{C} P^{\infty}$ of the bottom cell. The model will then be generalized to $D J_{K}(\underline{S})$, but without the need for an accompanying map. 
By definition, for $\sigma=\left(i_{1}, \ldots, i_{k}\right)$, let $S^{\sigma}=\prod_{j=1}^{k} S_{i_{j}}^{2}$, with the lower index recording coordinate position, and let $D J_{K}\left(S^{2}\right)=\bigcup_{\sigma \in K} S^{\sigma}$. Similarly, regarding $\mathbb{C} P^{\infty}$ as $B T$ where $T=S^{1}$, by definition $D J_{K}=\bigcup_{\sigma \in K} B T^{\sigma}$, where $B T^{\sigma}=B T_{i_{1}} \times \cdots \times B T_{i_{k}}$, again with the lower index recording coordinate position. Let $\imath^{\sigma}: S^{\sigma} \longrightarrow B T^{\sigma}$ be the product map $\prod_{j=1}^{k} \imath$. Then the map $D J_{K}(\underline{S}) \stackrel{D J_{K}(\imath)}{\longrightarrow} D J_{K}$ is, by definition, $\bigcup_{\sigma \in K} \imath^{\sigma}$.

Many useful properties of Adams-Hilton models were proved in $[\mathrm{AH}]$; a nice summary can be found in [An, 8.1]. First, an Adams-Hilton model of a $C W$-subspace can be extended to one for the whole space. Start with the inclusion of the bottom cell $S^{2} \stackrel{\imath}{\longrightarrow} \mathbb{C} P^{\infty}$. Then an Adams-Hilton model for $S^{2}$ can be extended to one for $\mathbb{C} P^{\infty}$. Second, an Adams-Hilton model for a product $A H(X \times Y)$ can be chosen so that it is quasi-isomorphic to $A(X) \otimes A(Y)$, and this respects the quasi-isomorphisms $\theta_{X \times Y}$ and $\theta_{X} \otimes \theta_{Y}$ to the respective cubical singular chain complexes. In our case, this lets us take the given model $A H\left(S^{2}\right)$ for $S^{2}$ and its extension $A H\left(\mathbb{C} P^{\infty}\right)$ for $\mathbb{C} P^{\infty}$ and produce a model for $S^{\sigma}$ mapping to $B T^{\sigma}$ which, up to quasi-isomorphisms, is $A H\left(S^{2}\right)^{\otimes \sigma}$ mapping factor-wise to $A H\left(\mathbb{C} P^{\infty}\right)^{\otimes \sigma}$. Third, Adams-Hilton models preserve colimits, given coherency conditions. That is, if $\left\{X_{\alpha}\right\}$ is a family of $C W$-subcomplexes of $X$ and $X=\bigcup_{\alpha} X_{\alpha}$, and there are models $A H\left(X_{\alpha}\right)$ satisfying the coherency conditions $\left.d_{V_{\alpha}}\right|_{A H\left(X_{\alpha} \cap X_{\beta}\right)}=\left.d_{V_{\beta}}\right|_{A H\left(X_{\alpha} \cap X_{\beta}\right)}$ and $\left.\theta_{X_{\alpha}}\right|_{A H\left(X_{\alpha} \cap X_{\beta}\right)}=\left.\theta_{X_{\beta}}\right|_{A H\left(X_{\alpha} \cap X_{\beta}\right)}$ for all pairs $(\alpha, \beta)$, then $\operatorname{colim}_{\alpha} A H\left(X_{\alpha}\right)$ is an Adams-Hilton model for $X$. In our case, we have $D J_{K}(\underline{S}) \stackrel{D J_{K}(\imath)}{\longrightarrow}$ $D J_{K}$ equalling, by definition, $\bigcup_{\sigma \in K} S^{\sigma} \stackrel{\bigcup_{\sigma \in K} \imath^{\sigma}}{\longrightarrow} \bigcup_{\sigma \in K} B T^{\sigma}$. Notice that the intersection $S^{\sigma_{1}} \cap S^{\sigma_{2}}$ is again a sub-product, namely $S^{\sigma_{1} \cap \sigma_{2}}$. Similarly, $B T^{\sigma_{1}} \cap B T^{\sigma_{2}}=B T^{\sigma_{1} \cap \sigma_{2}}$. Thus the compatibility of Adams-Hilton models with products implies that the coherency conditions will be satisfied for $\bigcup_{\sigma \in K} S^{\sigma}$ and $\bigcup_{\sigma \in K} B T^{\sigma}$, and for the map $\bigcup_{\sigma \in K} i^{\sigma}$. Hence there is a commutative diagram

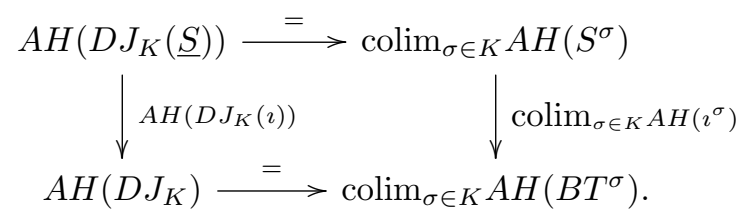

At this point one would like to say that homology commutes with colimits in order to describe $H_{*}\left(\Omega D J_{K}\left(S^{2}\right) ; \mathbb{Q}\right)$, say, as $\operatorname{colim}_{\sigma \in K} H_{*}\left(\Omega S^{\sigma} ; \mathbb{Q}\right)$. But there is a problem: $A H\left(D J_{K}(\underline{S})\right)$ is a noncommutative differential graded algebra and in general, colimits of such objects do not commute with homology. The point is that the colimit does not see higher bracket terms that may arise from the interaction of the differential and noncommutativity. Instead, one needs to take an appropriate homotopy colimit. However, in the case of directed $M F$-complexes, we are able to avoid this problem.

To see this, consider an analogue of (20) with respect to directed $M F$-complexes and fat wedges. To distinguish fat wedges, given $\sigma=\left(i_{1}, \ldots, i_{k}\right)$, let $F W\left(S^{2}, \sigma\right)$ be the fat wedge of $\prod_{j=1}^{k} S_{i_{j}}^{2}$, where the lower index refers to coordinate position. Let $F W(\sigma)$ be the fat wedge of $\prod_{j=1}^{k} \mathbb{C} P_{i_{j}}^{\infty}$. Let $\imath^{\sigma}: F W\left(S^{2}, \sigma\right) \longrightarrow F W(\sigma)$ be the map of fat wedges induced by $\imath$. Note that $F W\left(S^{2}, \sigma\right)=$ 
$\bigcup_{\tau \in\left(\Delta^{k}\right)_{k-1}} S^{\tau}$ and $F W(\sigma)=\bigcup_{\tau \in\left(\Delta^{k}\right)_{k-1}} B T^{\tau}$. Suppose $K$ is a directed $M F$-complex on $n$ vertices. The fact that $K$ is an $M F$-complex (missing face complex) implies that

$$
D J_{K}(\underline{S})=\bigcup_{\sigma \in M F(K)} F W\left(S^{2}, \sigma\right)=\bigcup_{\sigma \in M F(K)} \bigcup_{\tau \in\left(\Delta^{k}\right)_{k-1}} S^{\tau} .
$$

Since $K$ is a directed $M F$-complex, then in addition there is a sequence of subcomplexes $\emptyset \subseteq K_{1} \subseteq$ $\cdots \subseteq K_{l}=K$ where $K_{i}=K_{i-1} \cup \partial \sigma_{i}$ and $K_{i-1} \cap \partial \sigma_{i}$ is a face common to $K_{i-1}$ and $\partial \sigma_{i}$. Suppose this common face is $\left(t_{1}, \ldots, t_{l}\right)$. Then topologically there is a (strict) pushout

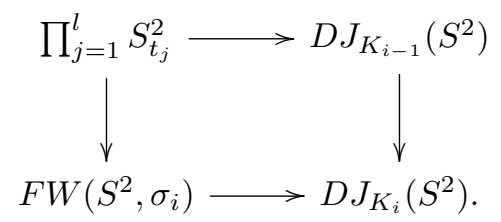

That is, $D J_{K_{i-1}}\left(S^{2}\right)$ and $F W\left(S^{2}, \sigma_{i}\right)$ have been glued together over the sub-product $\prod_{j=1}^{t} S_{t_{j}}^{2}$ of $\prod_{i=1}^{n} S^{2}$. This pushout satisfies the coherency conditions for the Adams-Hilton model. The same is true for $D J_{K}$ and the map $D J_{K}(\iota)$. Thus, in the case of directed $M F$-complexes, (20) can be reformulated as a commutative diagram

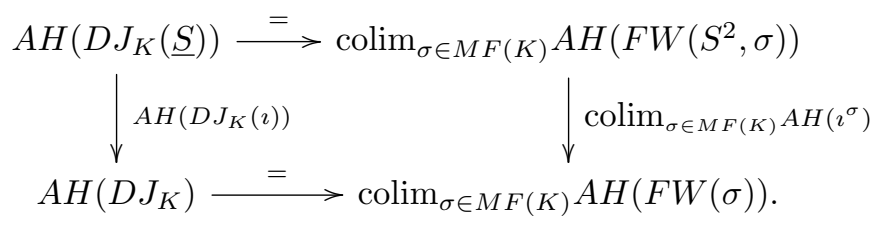

Since $K$ is a directed $M F$-complex, $K_{i}$ is obtained by gluing $K_{i-1}$ and $\partial \sigma_{i}$ along a common face. Thus the Adams-Hilton model $A H\left(D J_{K_{i}}\right)$ is a free extension of the differential graded algebras $A H\left(D J_{K_{i-1}}\right)$ and $A H\left(F W\left(\sigma_{i}\right)\right)$ and consequently, the diagram (21) is Reedy cofibrant (see [PR, Sections 3,4] for a discussion). Therefore, by [PR, Proposition 4.8] the colimits in (21) are naturally weakly equivalent to homotopy colimits. As homotopy colimits of differential graded algebras commute with homology, we immediately obtain the following.

Proposition 11.2. Let $K$ be a directed MF-complex. There is a commutative diagram of algebras

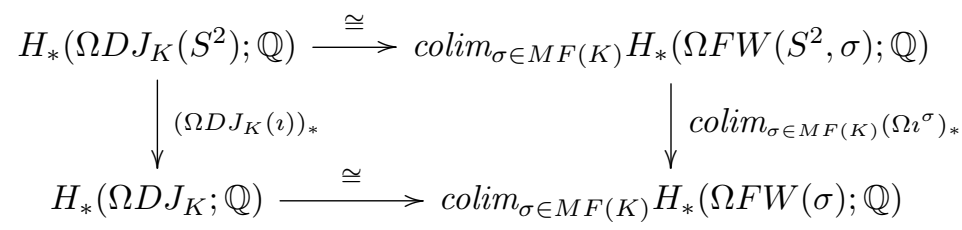

Proposition 11.2 reduces the problem of calculating $H_{*}\left(\Omega D J_{K}\left(S^{2}\right) ; \mathbb{Q}\right)$ and $H_{*}\left(\Omega D J_{K} ; \mathbb{Q}\right)$ to that of calculating the rational homology of looped fat wedges - with the proviso that the underlying model for the fat wedges must be compatible with the inclusion of sub-products. In our case we 
want more, that the homology of the looped fat wedges also keeps track of the Hurewicz images of adjointed higher Whitehead products. We will discuss this further in the next section.

Observe that the arguments above are equally valid for $D J_{K}(\underline{X})$, where $\underline{X}=\left\{X_{1}, \ldots, X_{m}\right\}$. The cases we particularly care about are $\underline{S}=\left\{S^{n_{1}+1}, \ldots, S^{n_{m}+1}\right\}$, with the special case of $D J_{K}\left(S^{2}\right)$, and the case of $D J_{K}$. The focus is on these cases as they give models which can be explicitly calculated. We do so for $D J_{K}(\underline{S})$ in Section 13 and $D J_{K}$ in Section 14. For future reference, we state the case for $\underline{S}$.

Proposition 11.3. Let $K$ be a directed $M F$-complex. An Adams-Hilton model for D $J_{K}(\underline{S})$ is

$$
A H\left(D J_{K}(\underline{S})\right)=\operatorname{colim}_{\sigma \in M F(K)} A H(F W(\sigma))
$$

and there is an isomorphism

$$
H_{*}\left(\Omega D J_{K}(\underline{S}) ; \mathbb{Q}\right) \cong \operatorname{colim}_{\sigma \in M F(K)} H_{*}(\Omega F W(\sigma) ; \mathbb{Q}) .
$$

\section{An Adams-Hilton model for $F W(\underline{S})$}

We are aiming for an Adams-Hilton model for $F W(\underline{S})$ over $\mathbb{Q}$ which is compatible with the inclusion of sub-products, and which keeps track of the Hurewicz images of adjointed higher Whitehead products. We will obtain one by using Allday's construction of a minimal Quillen model for $\pi_{*}(\Omega F W(\underline{S})) \otimes \mathbb{Q}$ and then using this to produce an Adams-Hilton model for $H_{*}(\Omega F W(\underline{S}) ; \mathbb{Q})$.

We begin with some general statements which hold for any path-connected space $X$. Assume from now on that the ground $\operatorname{ring} R$ is $\mathbb{Q}$. Observe that $\pi_{*}(X)$ can be given the structure of a graded Lie algebra by using the Whitehead product to define the bracket. Equivalently, by adjointing, $\pi_{*}(\Omega X)$ may be given the structure of a graded Lie algebra by using the Samelson product. Quillen [Q] associated to $X$ a free differential graded Lie algebra $\lambda(X)$ over $\mathbb{Q}$ with the property that there is an isomorphism $H_{*}(\lambda(X)) \longrightarrow \pi_{*}(\Omega X) \otimes \mathbb{Q}$. The free property of $\lambda(X)$ lets us write it as $L\left\langle V ; d_{V}\right\rangle$ for some graded $\mathbb{Q}$-module $V$ and differential $d_{V}$ on $V$. A Quillen model $M Q(X)$ is minimal if the differential has the property that $d(L\langle V\rangle) \subseteq[L\langle V\rangle, L\langle V\rangle]$.

Allday [Al] gave an explicit construction of a minimal Quillen model for $\pi_{*}(\Omega F W(\underline{S})) \otimes \mathbb{Q}$. This is stated in Theorem 12.1 once some notation has been introduced. The cells of $F W(\underline{S})$ are in one-to-one correspondence with sequences $\left(i_{1}, \ldots, i_{k}\right)$ where $1 \leq i_{1}<\cdots<i_{k} \leq m$ and $k<m$. The sequence $\left(i_{1}, \ldots, i_{k}\right)$ corresponds to the top cell of the coordinate subspace $S^{n_{i_{1}}+1} \times \cdots \times S^{n_{i_{k}}+1}$ within $F W(\underline{S})$. This cell has dimension $\sum_{s=1}^{k}\left(n_{i_{s}}+1\right)$. Note that the condition $k<m$ excludes only one sequence, $(1,2, \ldots, m)$, corresponding to the top cell of the product $S^{n_{1}+1} \times \cdots \times S^{n_{m}+1}$. Allday's minimal Quillen model for $\pi_{*}(\Omega F W(\underline{S})) \otimes \mathbb{Q}$ is of the form

$$
M Q(F W(\underline{S}))=L\left\langle V ; d_{V}\right\rangle
$$


where $V$ has one generator $b_{I}$ for each sequence $I=\left(i_{1}, \ldots, i_{k}\right)$ with $1 \leq i_{1}<\cdots<i_{k} \leq m$ and $k<m$, and the degree of $b_{I}$ is $\left(\sum_{s=1}^{k}\left(n_{i_{s}}+1\right)\right)-1$. Similarly, his minimal Quillen model for $\pi_{*}\left(\Omega \prod_{i=1}^{m} S^{n_{i}+1}\right) \otimes \mathbb{Q}$ is of the form

$$
M Q\left(\prod_{i=1}^{m} S^{n_{i}+1}\right)=L\left\langle W ; d_{W}\right\rangle
$$

where $W$ has one generator $b_{I}$ for each sequence $I=\left(i_{1}, \ldots, i_{k}\right)$ with $1 \leq i_{1}<\cdots<i_{k} \leq m$, and the degree of $b_{I}$ is $\left(\Sigma_{s=1}^{k}\left(n_{i_{s}}+1\right)\right)-1$.

To describe the differentials $d_{V}$ and $d_{W}$, fix a sequence $I=\left(i_{1}, \ldots, i_{k}\right)$ where $1 \leq i_{1}<\cdots<i_{k} \leq$ $m$ and $k \geq 2$. If $k<m$ this corresponds to a generator $b_{I}$ of $V$, and if $k \leq m$ this corresponds to a generator $b_{I}$ of $W$. In either case, the degree of $b_{I}$ is $\left|b_{I}\right|=\left(\sum_{s=1}^{k} n_{i_{s}}+1\right)-1$. Let $\mathcal{S}_{I}$ be the collection of all shuffles $\left(J, J^{\prime}\right)$ of $\left\{i_{1}, \ldots, i_{k}\right\}$ with the property that $j_{1}=1$ (known as a type II shuffle relative to 1$)$. If $\left(J, J^{\prime}\right)$ is an $(r, s)$-shuffle of $\{1, \ldots, k\}$, let $\epsilon\left(J, J^{\prime}\right) \in\{0,1\}$ be the number determined by the equation $z_{i_{1}} \cdots z_{i_{k}}=(-1)^{\epsilon\left(J, J^{\prime}\right)} z_{j_{1}} \cdots z_{j_{r}} z_{j_{1}^{\prime}} \cdots z_{j_{s}^{\prime}}$ in the graded rational symmetric algebra generated by $z_{i_{1}}, \ldots, z_{i_{k}}$ with $\left|z_{i_{t}}\right|=n_{i_{t}}+1$ for $1 \leq t \leq k$. Let

$$
a_{I}=-\Sigma_{\left(J, J^{\prime}\right) \in \mathcal{S}_{\mathcal{I}}}(-1)^{\left|b_{I}\right|+\epsilon\left(J, J^{\prime}\right)}\left[b_{J}, b_{J^{\prime}}\right] .
$$

As special cases, let $b=b_{(1, \ldots, m)}$ and $a=a_{(1, \ldots, m)}$.

Theorem 12.1. With $V$ and $W$ as defined above, minimal Quillen models $L\left\langle V ; d_{V}\right\rangle$ and $L\left\langle W ; d_{W}\right\rangle$ for $\pi_{*}(\Omega F W(\underline{S})) \otimes \mathbb{Q}$ and $\pi_{*}\left(\prod_{i=1}^{n} \Omega S^{m_{i}+1}\right) \otimes \mathbb{Q}$ can be chosen to satisfy the following properties:

(a) $W=V \oplus\{b\}$;

(b) $d_{V}\left(b_{I}\right)=0$ if $I=(i)$ for $1 \leq i \leq m$;

(c) $d_{V}\left(b_{I}\right)=a_{I}$ for $I=\left(i_{1}, \ldots, i_{k}\right)$ with $2 \leq k<m$;

(d) $d_{W}$ restricted to $V$ is $d_{V}$;

(e) $d_{W}(b)=a$;

(f) the adjoint of the higher order Whitehead product $S^{|b|-1} \stackrel{\phi_{m}}{\longrightarrow} F W(\underline{S})$ which attaches the top cell to the product $\prod_{i=1}^{m} S^{n_{i}+1}$ is homotopic to a.

There is an explicit map $\alpha: L\langle V\rangle \longrightarrow \pi_{*}(\Omega F W(\underline{S}))$. Let $b_{I} \in V$ for $I=\left(i_{1}, \ldots, i_{k}\right)$. This corresponds to the top cell of the coordinate subspace $S^{n_{i_{1}}+1} \times \cdots \times S^{n_{i_{k}}+1}$ in $F W(\underline{S})$. Let $F W\left(i_{1}, \ldots, i_{k}\right)$ be the fat wedge in $S^{n_{i_{1}}+1} \times \cdots \times S^{n_{i_{k}}+1}$. Let $\alpha\left(b_{I}\right)$ be the adjoint of the composite $S^{\left|b_{I}\right|-1} \stackrel{\phi_{k}}{\longrightarrow} F W\left(i_{1}, \ldots, i_{k}\right) \longrightarrow \prod_{j=1}^{k} S^{n_{i_{j}}+1} \longrightarrow F W(\underline{S})$, where the latter two maps are the inclusions. Now extend $\alpha$ to $L\langle V\rangle$ by using the fact that $\pi_{*}(\Omega F W(\underline{S})) \otimes \mathbb{Q}$ is a Lie algebra under the Samelson product. Allday's statement that $L\left\langle V ; d_{V}\right\rangle$ is a minimal Quillen model for $\pi_{*}(\Omega F W(\underline{S})) \otimes \mathbb{Q}$ says two things: first, that $\alpha$ can be upgraded from a map of Lie algebras to a map of differential graded Lie algebras, where the differential on $\pi_{*}(\Omega F W(\underline{S})) \otimes \mathbb{Q}$ is zero, and 
second, that this upgraded map induces an isomorphism in homology. A similar construction can be made with respect to $\prod_{i=1}^{m} S^{n_{i}+1}$.

We now pass from a minimal Quillen model to an Adams-Hilton model. In general, observe that the Hurewicz homomorphism $\pi_{*}(\Omega X) \otimes \mathbb{Q} \longrightarrow H_{*}(\Omega X ; \mathbb{Q})$ factors as the composite $\pi_{*}(\Omega X) \otimes \mathbb{Q} \stackrel{c}{\longrightarrow}$ $C U_{*}(\Omega X) \stackrel{h}{\longrightarrow} H_{*}(\Omega X ; \mathbb{Q})$, where $C U_{*}(\Omega X)$ is the cubical singular chain complex with coefficients in $\mathbb{Q}, c$ is the canonical map to the cubical singular chains, and $h$ is the quotient map to the homology of the chain complex. Note that $h$ is an algebra map. Let $M Q(X)$ be a minimal Quillen model for $\pi_{*}(\Omega X) \otimes \mathbb{Q}$, and suppose there is an associated map of differential graded Lie algebras $\alpha: M Q(X)=$ $L\left\langle V_{X} ; d_{V_{X}}\right\rangle \longrightarrow \pi_{*}(\Omega X) \otimes \mathbb{Q}$ which induces an isomorphism in homology. Since $C U_{*}(\Omega X)$ is a differential graded algebra, the composite $c \circ \alpha$ extends to a map $\theta_{X}: U L\left\langle V_{X} ; d_{V_{X}}\right\rangle \longrightarrow C U_{*}(\Omega X)$ of differential graded algebras. Thus there is a commutative diagram

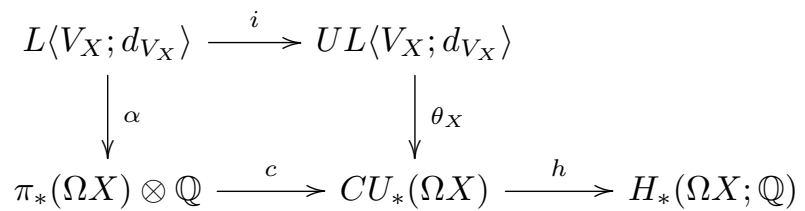

where $i$ is the inclusion. By Milnor-Moore [MM], regarding $\pi_{*}(\Omega X) \otimes \mathbb{Q}$ as a Lie algebra, we have $H_{*}(\Omega X ; \mathbb{Q}) \cong U\left(\pi_{*}(\Omega X) \otimes \mathbb{Q}\right)$, with the isomorphism induced by the Hurewicz homomorphism. On the other hand, $h$ is a map of differential graded algebras once $H_{*}(\Omega X ; \mathbb{Q})$ has been given the zero differential. Thus $h \circ \theta_{X}$ is a map of differential graded algebras, and therefore it is determined by its restriction to the generating set $V$. The commutativity of the diagram implies that $\left.h \circ \theta_{X}\right|_{V}=\left.h \circ c \circ \alpha\right|_{V}=: q$. Thus $h \circ \theta_{X}=U(q)$, implying that $h \circ \theta_{X}$ induces an isomorphism in rational homology. Hence $U L\left\langle V_{X} ; d_{V_{X}}\right\rangle$ together with the quasi-isomorphism $\theta_{X}$ is an Adams-Hilton model for $X$.

In our case, we obtain Adams-Hilton models $\left(U L\left\langle V ; d_{V}\right\rangle, \theta_{F W}\right)$ and $\left(U L\left\langle W ; d_{W}\right\rangle, \theta_{\Pi}\right)$ for $F W(\underline{S})$ and $\prod_{i=1}^{m} S^{n_{i}+1}$, respectively. Theorem 12.1 therefore implies the following.

Theorem 12.2. The Adams-Hilton models $A H(F W(\underline{S}))=\left(U L\left\langle V ; d_{V}\right\rangle, \theta_{F W}\right)$ and $A H\left(\prod_{i=1}^{m} S^{n_{i}+1}\right)=$ $\left(U L\left\langle W ; d_{W}\right\rangle, \theta_{\Pi}\right)$ have the following properties:

(a) $W=V \oplus\{b\}$;

(b) $d_{V}\left(b_{I}\right)=0$ if $I=(i)$ for $1 \leq i \leq m$;

(c) $d_{V}\left(b_{I}\right)=a_{I}$ for $I=\left(i_{1}, \ldots, i_{k}\right)$ with $2 \leq k<m$;

(d) $d_{W}$ restricted to $A H(F W(\underline{S}))$ is $d_{V}$;

(e) $d_{W}(b)=a$;

(f) the adjoint of the higher order Whitehead product $S^{|b|-1} \stackrel{\phi_{m}}{\longrightarrow} F W(\underline{S})$ which attaches the top cell to the product $\prod_{i=1}^{m} S^{n_{i}+1}$ has "Hurewicz" image a. 
Remark 12.3. The inductive definition of the differential $d_{V}$ in the minimal Quillen model $L\left\langle V ; d_{V}\right\rangle$ for $F W(\underline{S})$ in Theorem 12.2 implies that the differential is compatible with the inclusion of subproducts. The same is therefore true in $U L\left\langle V ; d_{V}\right\rangle$. Moreover, the differential $d_{V}$ is what turns the map $\alpha$ into a map of differential graded Lie algebras, and so both $\alpha$ and its extension to the quasi-isomorphism $\theta_{F W}$ are compatible with the inclusion of sub-products.

As well as the inductive nature of the Adams-Hilton model, Theorem 12.2 also explicitly identifies the "Hurewicz" image of the adjoint of the higher order Whitehead product $\phi_{m}$. We put Hurewicz in quotes as this image is an element in an Adams-Hilton model, whereas the honest Hurewicz image is obtained after taking homology. That is, $a$ is a cycle in $A H(F W(\underline{S}))$, which could also be a boundary. This turns out not to be the case. Observe that there is a sequence of isomorphisms $H_{*}(A H(F W(\underline{S}))) \cong H_{*}\left(U L\left\langle V ; d_{V}\right\rangle\right) \cong U\left(H_{*}\left(L\left\langle V ; d_{V}\right\rangle\right)\right)$, since homology commutes with the universal enveloping algebra functor. To calculate $H_{*}\left(L\left\langle V ; d_{V}\right\rangle\right)$ we proceed exactly as in [B], where Bubenik used separated Lie models to elegantly obtain the answer. This is stated in Theorem 12.4 in terms of the universal enveloping algebra rather than the Lie algebra as we are ultimately after $H_{*}(\Omega F W(\underline{S}) ; \mathbb{Q})$. To state the result we need to introduce more notation. For $1 \leq i \leq m$, let $b_{i}$ be the generator in $V$ (or $W$ ) corresponding to the sequence $I=(i)$. That is, $b_{i}$ corresponds to the cell $S^{n_{i}+1}$ in $S^{n_{1}+1} \times \cdots \times S^{n_{m}+1}$. Let $N=\left(\sum_{i=1}^{k} n_{i}+1\right)-2$. Recall from Section (8) that, $L_{d s}\left\langle b_{1}, \ldots, b_{m}\right\rangle=\oplus_{i=1}^{m} L\left\langle b_{i}\right\rangle$. Observe that $H_{*}\left(A H\left(\prod_{j=1}^{k} S^{n_{j}+1}\right)\right) \cong U L_{d s}\left\langle b_{1}, \ldots, b_{m}\right\rangle$.

Theorem 12.4. For $m \geq 3$, there are algebra isomorphisms

$$
H_{*}(\Omega F W(\underline{S}) ; \mathbb{Q}) \cong H_{*}(A H(F W(\underline{S}))) \cong U\left(L_{d s}\left\langle b_{1}, \ldots, b_{m}\right\rangle \coprod L\langle u\rangle\right)
$$

where $u$, of degree $N$, is the Hurewicz image of the adjoint of the higher Whitehead product $\phi_{m}$. Further, the looped inclusion $\Omega F W(\underline{S}) \longrightarrow \prod_{i=1}^{m} \Omega S^{n_{i}+1}$ is modelled by the map

$$
U\left(L_{d s}\left\langle b_{1}, \ldots, b_{m}\right\rangle \amalg L\langle u\rangle\right) \stackrel{U(\pi)}{\longrightarrow} U L_{d s}\left\langle b_{1}, \ldots, b_{m}\right\rangle
$$

where $\pi$ is the projection.

Note that the calculation of $H_{*}(\Omega F W(\underline{S}) ; \mathbb{Q})$ is not new, it was originally done by Lemaire [Le]. What is new and important to keep in mind about Theorem 12.4 is that the calculation also keeps track of the Hurewicz image of the adjointed higher Whitehead product $\phi_{m}$.

Remark 12.5. When $m=2$, we have $F W(\underline{S})=S^{n_{1}+1} \vee S^{n_{2}+1}$ and then it is well known that $H_{*}(\Omega F W(\underline{S}) ; \mathbb{Q}) \cong H_{*}\left(\Omega\left(S^{n_{1}+1} \vee S^{n_{2}+1}\right) ; \mathbb{Q}\right) \cong U L\left\langle b_{1}, b_{2}\right\rangle$. In this case $\phi_{2}$ is the ordinary Whitehead product and its adjoint has Hurewicz image $u=\left[b_{1}, b_{2}\right]$. In this case we can regard $L\left\langle b_{1}, b_{2}\right\rangle$ as $L_{d s}\left\langle b_{1}, b_{2}\right\rangle \amalg L\langle u\rangle$, modulo Jacobi identities on brackets of the form $[u,-]=\left[\left[b_{1}, b_{2}\right],-\right]$. 


\section{Properties of $\Omega D J_{K}(\underline{S})$ AND $\Omega \mathcal{Z}_{K}(\underline{S})$ FOR DiRECTED $M F$-COMPLEXES}

In this section we explicitly calculate $H_{*}\left(\Omega D J_{K}(\underline{S}) ; \mathbb{Q}\right)$ when $K$ is a directed $M F$-complex, proving Theorem 8.5. This is then used in tandem with the loops on the homotopy fibration $\mathcal{Z}_{K}(\underline{S}) \stackrel{f}{\longrightarrow} D J_{K}(\underline{S}) \stackrel{g}{\longrightarrow} \prod_{i=1}^{m} S^{n_{i}+1}$ to calculate $H_{*}\left(\Omega \mathcal{Z}_{K}(\underline{S}) ; \mathbb{Q}\right)$. We then give a homotopy decomposition of $\mathcal{Z}_{K}(\underline{S})$ as a wedge of spheres and describe the map $\mathcal{Z}_{K}(\underline{S}) \longrightarrow D J_{K}(\underline{S})$ in terms of higher Whitehead products and iterated Whitehead products, proving Theorem 8.7.

Remark 13.1. To simplify the presentation, for the remainder of Sections 13 and 14 we will assume that the given directed $M F$-complex $K$ has the property that $|\sigma|>2$ for every $\sigma \in M F(K)$. This is to appeal directly to Theorem 12.4. If $|\sigma|=2$ for some $\sigma=\left(i_{1}, i_{2}\right) \in M F(K)$, then the calculations can be modified by regarding $L\left\langle b_{i_{1}}, b_{i_{2}}\right\rangle$ as $L_{d s}\left\langle b_{i_{1}}, b_{i_{2}}\right\rangle \coprod L\langle u\rangle$ for $u=\left[b_{i_{1}}, b_{i_{2}}\right]$ as in Remark 12.5, and by introducing the ideal $J$ discussed in Section 8.

We begin by calculating $H_{*}\left(\Omega D J_{K}(\underline{S}) ; \mathbb{Q}\right)$ using the Adams-Hilton model

$$
A H\left(D J_{K}(\underline{S})\right)=\operatorname{colim}_{\sigma \in M F(K)} A H(F W(\sigma))
$$

in Proposition 11.3. Let $b_{1}, \ldots, b_{m}$ be the generators in $A H\left(D J_{K}(\underline{S})\right)$ corresponding to the cells $S^{n_{1}+1}, \ldots, S^{n_{m}+1}$ respectively. For $\sigma=\left(i_{1}, \ldots, i_{k}\right) \in K$, observe that $\left\{b_{i_{1}}, \ldots, b_{i_{k}}\right\}$ corresponds to the cells $S^{n_{i}+1}$ which are in $F W(\sigma)$. Let $N_{\sigma}=\left(\sum_{j=1}^{k} n_{i_{j}}+1\right)-2$. By Theorem 12.4, we have $H_{*}(A H(F W(\sigma))) \cong U\left(L_{d s}\left\langle b_{i_{1}}, \ldots, b_{i_{k}}\right\rangle \coprod L\left\langle u_{\sigma}\right\rangle\right)$ where $u_{\sigma}$ is the Hurewicz image of the adjoint of a higher Whitehead product $S^{N_{\sigma}+1} \longrightarrow F W(\sigma)$.

We now prove Theorem 8.5, restated below to match the simplifying assumption in Remark 13.1.

Theorem 13.2. Let $K$ be a directed $M F$-complex such that $|\sigma|>2$ for every $\sigma \in M F(K)$. There is an algebra isomorphism

$$
H_{*}\left(\Omega D J_{K}(\underline{S}) ; \mathbb{Q}\right) \cong U\left(L_{d s}\left\langle b_{1}, \ldots, b_{m}\right\rangle \coprod L\left\langle u_{\sigma} \mid \sigma \in M F(K)\right\rangle\right)
$$

where each $u_{\sigma}$ is the Hurewicz image of the adjoint of a higher Whitehead product. Further, the loop map $\Omega D J_{K}(\underline{S}) \longrightarrow \prod_{i=1}^{m} \Omega S^{n_{i}+1}$ is modelled by the map

$$
U\left(L_{d s}\left\langle b_{1}, \ldots, b_{m}\right\rangle \coprod L\left\langle u_{\sigma} \mid \sigma \in M F(K)\right\rangle\right) / J \stackrel{U(\pi)}{\longrightarrow} U L_{d s}\left\langle b_{1}, \ldots, b_{m}\right\rangle
$$

where $\pi$ is the projection.

Proof. Consider the string of isomorphisms

$$
\begin{aligned}
H_{*}\left(\Omega D J_{K}(\underline{S}) ; \mathbb{Q}\right) & \cong H_{*}\left(A H\left(D J_{K}(\underline{S})\right)\right. \\
& \cong \operatorname{colim}_{\sigma \in M F(K)} H_{*}(A H(F W(\sigma))) \\
& \cong \operatorname{colim}_{\sigma \in M F(K)} U\left(L_{d s}\left\langle b_{i_{1}}, \ldots, b_{i_{k}}\right\rangle \coprod L\left\langle u_{\sigma}\right\rangle\right) \\
& \cong U\left(\operatorname{colim}_{\sigma \in M F(K)} L_{d s}\left\langle b_{i_{1}}, \ldots, b_{i_{k}}\right\rangle \coprod L\left\langle u_{\sigma}\right\rangle\right) \\
& \cong U\left(L_{d s}\left\langle b_{1}, \ldots, b_{m}\right\rangle \coprod L\left\langle u_{\sigma} \mid \sigma \in M F(K)\right\rangle\right) .
\end{aligned}
$$


The first isomorphism holds because $A H\left(D J_{K}(\underline{S})\right)$ is an Adams-Hilton model. The second isomorphism holds because by Proposition 11.3. The third isomorphism holds by Theorem 12.4. For the fourth isomorphism, Remark 12.3 implies that in the case of directed $M F$-complexes (when the missing faces are glued along common faces, which topologically correspond to sub-products in $D J_{K}(\underline{S})$ ) the calculation of $H_{*}(A H(F W(\sigma))) \cong U\left(L_{d s}\left\langle b_{i_{1}}, \ldots, b_{i_{k}}\right\rangle\right)$ is compatible with the inclusion of subproducts. Therefore both the underlying Lie algebra and its universal enveloping algebra respect the colimit over $M F(K)$, and so the fourth isomorphism holds. Provided the fifth isomorphism holds, the string of isomorphisms establishes the isomorphism asserted by the theorem. The statement regarding Hurewicz images now follows from that in Theorem 12.4. The statement regarding the model for the looped map $\Omega D J_{K}(\underline{S}) \longrightarrow \prod_{i=1}^{m} \Omega S^{n_{i}+1}$ follows again from Remark 12.3 regarding the compatibility of the colimit with the inclusion of sub-products.

It remains to establish the fifth isomorphism in the string of isomorphisms above. This is really a statement about Lie algebras, so we will show that

$$
\operatorname{colim}_{\sigma \in M F(K)} L_{d s}\left\langle b_{i_{1}}, \ldots, b_{i_{k}}\right\rangle \coprod L\left\langle u_{\sigma}\right\rangle \cong L_{d s}\left\langle b_{1}, \ldots, b_{m}\right\rangle \coprod L\left\langle u_{\sigma} \mid \sigma \in M F(K)\right\rangle .
$$

Since the set $M F(K)$ of minimal missing faces of $K$ is finite, the colimit can be rewritten as a free coproduct modulo relations. To indicate the dependence on $\sigma=\left(i_{1}, \ldots, i_{k}\right)$, write $L_{d s}\left\langle b_{i_{1}}, \ldots, b_{i_{k}}\right\rangle \amalg L\left\langle u_{\sigma}\right\rangle$ as $L_{d s}\left\langle b_{i_{1}}^{\sigma}, \ldots, b_{i_{k}}^{\sigma}\right\rangle \coprod L\left\langle u_{\sigma}\right\rangle$. Rearranging terms, there is an isomorphism of free coproducts

$$
\coprod_{\sigma \in M F(K)}\left(L_{d s}\left\langle b_{i_{1}}^{\sigma}, \ldots, b_{i_{k}}^{\sigma}\right\rangle \coprod L\left\langle u_{\sigma}\right\rangle\right) \cong\left(\coprod_{\sigma \in M F(K)} L_{d s}\left\langle b_{i_{1}}^{\sigma}, \ldots, b_{i_{k}}^{\sigma}\right\rangle\right) \coprod L\left\langle u_{\sigma} \mid \sigma \in M F(K)\right\rangle .
$$

Thus there is an isomorphism

$$
\begin{aligned}
& \operatorname{colim}_{\sigma \in M F(K)} L_{d s}\left\langle b_{i_{1}}^{\sigma}, \ldots, b_{i_{k}}^{\sigma}\right\rangle \coprod L\left\langle u_{\sigma}\right\rangle \cong \\
&\left(\left(\coprod_{\sigma \in M F(K)} L_{d s}\left\langle b_{i_{1}}^{\sigma}, \ldots, b_{i_{k}}^{\sigma}\right\rangle\right) \coprod L\left\langle u_{\sigma} \mid \sigma \in M F(K)\right\rangle\right) / \sim
\end{aligned}
$$

where the relations $\sim$ are as follows. By definition of a directed $M F$-complex, two minimal missing faces $\sigma, \sigma^{\prime} \in M F(K)$ intersect along a common proper face, which we label as $\tau$. If $\tau=\emptyset$, then as subspaces of $D J_{K}(\underline{S}), F W(\sigma)$ and $F W\left(\sigma^{\prime}\right)$ intersect only at the basepoint. The Adams-Hilton model of $F W(\sigma) \vee F W\left(\sigma^{\prime}\right)$ is therefore the coproduct of the Adams-Hilton models for each individual space, so after taking homology no relation is introduced on the underlying Lie algebras in (23). If $\tau \neq \emptyset$, suppose that $\tau=\left(r_{1}, \ldots, r_{s}\right)$. Topologically, this intersection corresponds to the inclusion of the proper subproduct $\prod_{t=1}^{s} S^{2}$ into $D J_{K}(\underline{S})$. The Adams-Hilton model for the loop space of this subproduct has homology isomorphic to $U L\left\langle b_{r_{1}}^{\tau}, \ldots, b_{r_{s}}^{\tau}\right\rangle$, and by Remark 12.3 the inclusion of this subproduct is compatible with the Adams-Hilton models for both $\Omega F W(\sigma)$ and $\Omega F W\left(\sigma^{\prime}\right)$. Therefore, upon taking homology, in $U\left(L_{d s}\left\langle b_{i_{1}}^{\sigma}, \ldots, b_{i_{k}}^{\sigma}\right\rangle \amalg L\left\langle u_{\sigma}\right\rangle\right)$ and $U\left(L_{d s}\left\langle b_{i_{1}}^{\sigma^{\prime}}, \ldots, b_{i_{k^{\prime}}}^{\sigma^{\prime}}\right\rangle \amalg L\left\langle u_{\sigma^{\prime}}\right\rangle\right)$ we have $b_{r_{t}}^{\sigma}=b_{r_{t}}^{\sigma^{\prime}}$ for every $r_{1}, \ldots, r_{t}$. This relation holds equally well on the underlying Lie algebras. 
Hence the relation $\sim$ in (23) is given by identifying $b_{r_{\ell}}^{\sigma}$ and $b_{r_{\ell}}^{\sigma^{\prime}}$ whenever $r_{\ell} \in \sigma \cap \sigma^{\prime}$. Consequently, considering all the minimal missing faces of $K$, we obtain

$$
\begin{gathered}
\left(\left(\coprod_{\sigma \in M F(K)} L_{d s}\left\langle b_{i_{1}}^{\sigma}, \ldots, b_{i_{k}}^{\sigma}\right\rangle\right) \coprod L\left\langle u_{\sigma} \mid \sigma \in M F(K)\right\rangle\right) / \sim \cong \\
L_{d s}\left\langle b_{1}, \ldots, b_{m}\right\rangle \coprod L\left\langle u_{\sigma} \mid \sigma \in M F(K)\right\rangle .
\end{gathered}
$$

Theorem 13.2 is the crucial algebraic result. We first use it to determine $H_{*}\left(\Omega \mathcal{Z}_{K}(\underline{S}) ; \mathbb{Q}\right)$, and then to determine a more detailed description of the Hurewicz homomorphism.

Since $L_{d s}\left\langle b_{1}, \ldots, b_{m}\right\rangle \coprod L\left\langle u_{\sigma} \mid \sigma \in M F(K)\right\rangle$ is a coproduct, there is a short exact sequence of graded Lie algebras

$$
L\langle R\rangle \stackrel{i}{\longrightarrow} L_{d s}\left\langle b_{1}, \ldots, b_{m}\right\rangle \coprod L\left\langle u_{\sigma} \mid \sigma \in M F(K)\right\rangle \stackrel{\pi}{\longrightarrow} L_{d s}\left\langle b_{1}, \ldots, b_{m}\right\rangle
$$

where $i$ is the inclusion, $\pi$ is the projection, and

$$
R=\left\{\left[\left[u_{\sigma}, b_{j_{1}}\right], \ldots, b_{j_{l}}\right] \mid \sigma \in M F(K), 1 \leq j_{1} \leq \cdots \leq j_{l} \leq m, 0 \leq l<\infty\right\}
$$

Here, when $l=0$ we interpret the bracket as simply being $u_{\sigma}$.

Proposition 13.3. There is a commutative diagram of algebras

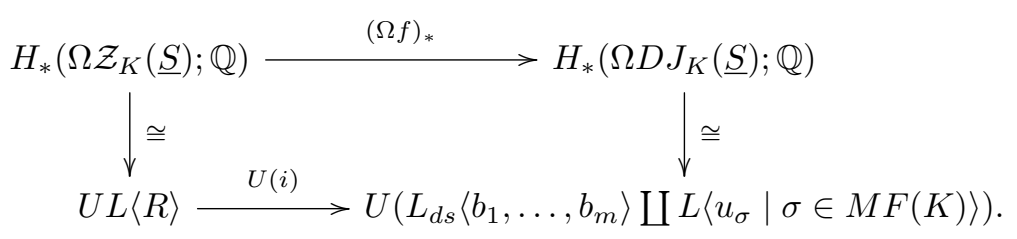

Proof. By [CMN, 3.7], a short exact sequence of graded Lie algebras induces a short exact sequence of Hopf algebras. In our case, (24) induces a short exact sequence

$$
U L\langle R\rangle \stackrel{U(i)}{\longrightarrow} U\left(L_{d s}\left\langle b_{1}, \ldots, b_{m}\right\rangle \coprod L\left\langle u_{\sigma} \mid \sigma \in M F(K)\right\rangle\right) \stackrel{U(\pi)}{\longrightarrow} U L_{d s}\left\langle b_{1}, \ldots, b_{m}\right\rangle .
$$

Here, by a short exact sequence of Hopf algebras, we mean that there is an isomorphism

$$
U\left(L_{d s}\left\langle b_{1}, \ldots, b_{m}\right\rangle \amalg L\left\langle u_{\sigma} \mid \sigma \in M F(K)\right\rangle\right) \cong U L_{d s}\left\langle b_{1}, \ldots, b_{m}\right\rangle \otimes U L\langle R\rangle
$$

as right $U L\langle R\rangle$-modules and left $U L_{d s}\left\langle b_{1}, \ldots, b_{m}\right\rangle$-comodules. In particular, $U(i)$ is the algebra kernel of $U(\pi)$. On the other hand, Theorem 13.2 implies that $U(\pi)$ is a model for the looped inclusion $\Omega D J_{K}(\underline{S}) \stackrel{\Omega g}{\longrightarrow} \prod_{i=1}^{m} \Omega S^{n_{i}+1}$. Since $\Omega g$ has a right homotopy inverse, the homotopy decomposition $\Omega D J_{K}(\underline{S}) \simeq\left(\prod_{i=1}^{m} \Omega S^{n_{i}+1}\right) \times \Omega \mathcal{Z}_{K}(\underline{S})$ implies that there is a short exact sequence of Hopf algebras $H_{*}\left(\Omega \mathcal{Z}_{K}(\underline{S}) ; \mathbb{Q}\right) \longrightarrow H_{*}\left(\Omega D J_{K}(\underline{S}) ; \mathbb{Q}\right) \stackrel{U(\pi)}{\longrightarrow} H_{*}\left(\prod_{i=1}^{m} \Omega S^{n_{i}+1} ; \mathbb{Q}\right)$. Thus $H_{*}\left(\Omega \mathcal{Z}_{K}(\underline{S}) ; \mathbb{Q}\right)$ is also the algebra kernel of $U(\pi)$, and the proposition follows. 
Theorem 13.2 implies that the rational homology of $\Omega D J_{K}(\underline{S})$ is generated by Hurewicz images. To be precise, suppose that $\sigma=\left(i_{1}, \ldots, i_{k}\right) \in M F(K)$. Recall that $\underline{S}=\left(S^{n_{i_{1}}+1}, \ldots, S^{n_{i_{k}}+1}\right)$, and let $N_{\sigma}=\left(\sum_{j=1}^{k} n_{i_{j}+1}\right)-2$. Let

$$
w_{\sigma}: S^{N_{\sigma}+1} \stackrel{\phi_{k}}{\longrightarrow} F W(\sigma) \longrightarrow D J_{K}(\underline{S})
$$

be the higher Whitehead product. Let

$$
s_{\sigma}: S^{N_{\sigma}} \longrightarrow \Omega D J_{K}(\underline{S})
$$

be the adjoint of $w_{\sigma}$. As stated in Theorem 13.2, the element $u_{\sigma} \in H_{*}\left(\Omega D J_{K}(\underline{S}) ; \mathbb{Q}\right)$ is the Hurewicz image of $s_{\sigma}$. For $1 \leq i \leq m$, let

$$
a_{i}: S^{n_{i}+1} \longrightarrow D J_{K}(\underline{S})
$$

be the coordinate inclusion and let

$$
\bar{a}_{i}: S^{n_{i}} \longrightarrow \Omega D J_{K}(\underline{S})
$$

be the adjoint of $a_{i}$. The Hurewicz image of $\bar{a}_{i}$ is $b_{i}$. Let $\mathcal{I}$ be the index set for $R$ introduced in (25). Then $\alpha \in \mathcal{I}$ corresponds to a missing face $\sigma \in M F(K)$ and a sequence $\left(j_{1}, \ldots, j_{l}\right)$ where $1 \leq j_{1} \leq \cdots \leq j_{l} \leq m$ and $0 \leq l<\infty$. Given such an $\alpha$, let $t_{\alpha}=\left(\sum_{t=1}^{l} n_{j_{t}}\right)+N_{\sigma}$. Then there is a Samelson product

$$
\left[\left[s_{\sigma}, \bar{a}_{j_{1}}\right], \ldots, \bar{a}_{j_{l}}\right]: S^{t_{\alpha}} \longrightarrow \Omega D J_{K}(\underline{S})
$$

Since Samelson products commute with Hurewicz images, the Hurewicz image of $\left[\left[s_{\sigma}, \bar{a}_{j_{1}}\right], \ldots, \bar{a}_{j_{l}}\right]$ is $\left[\left[u_{\sigma}, b_{j_{1}}\right], \ldots, b_{j_{l}}\right]$. Adjointing, we have a Whitehead product

$$
\left[\left[w_{\sigma}, a_{j_{1}}\right], \ldots, a_{j_{l}}\right]: S^{t_{\alpha}+1} \longrightarrow D J_{K}(\underline{S}) .
$$

Taking the wedge sum over all possible $\alpha$, we obtain a map

$$
W: \bigvee_{\alpha \in \mathcal{I}} S^{t_{\alpha}+1} \longrightarrow D J_{K}(\underline{S})
$$

Corollary 13.4. The map $\Omega\left(\bigvee_{\alpha \in \mathcal{I}} S^{t_{\alpha}+1}\right) \stackrel{\Omega W}{\longrightarrow} \Omega D J_{K}(\underline{S})$ induces in rational homology the map $U L\langle R\rangle \stackrel{U(i)}{\longrightarrow} U\left(L_{d s}\left\langle b_{1}, \ldots, b_{m}\right\rangle \coprod L\left\langle u_{\sigma} \mid \sigma \in M F(K)\right\rangle\right)$.

Proof. Let $S$ be the composite

$$
S: \bigvee_{\alpha \in \mathcal{I}} S^{t_{\alpha}} \stackrel{E}{\longrightarrow} \Omega\left(\bigvee_{\alpha \in \mathcal{I}} S^{t_{\alpha}+1}\right) \stackrel{\Omega W}{\longrightarrow} \Omega D J_{K}(\underline{S}) .
$$

Then $S$ is homotopic to the adjoint of $W$. In particular, the wedge summands of $S$ are the Samelson products $\left[\left[s_{\sigma}, \bar{a}_{j_{1}}\right], \ldots, \bar{a}_{j_{l}}\right]$ for $\alpha \in \mathcal{I}$. Thus, taking Hurewicz images, $S_{*}$ is the composite

$$
\bar{i}: R \hookrightarrow U L\langle R\rangle \stackrel{U(i)}{\longrightarrow} U\left(L_{d s}\left\langle b_{1}, \ldots, b_{m}\right\rangle \coprod L\left\langle u_{\sigma} \mid \sigma \in M F(K)\right\rangle\right) \stackrel{\cong}{\longrightarrow} H_{*}\left(\Omega D J_{K}(\underline{S})\right) .
$$


By the Bott-Samelson theorem, $H_{*}\left(\Omega\left(\bigvee_{\alpha \in \mathcal{I}} S^{t_{\alpha}+1}\right) ; \mathbb{Q}\right) \cong T\left(\widetilde{H}_{*}\left(\bigvee_{\alpha \in \mathcal{I}} S^{t_{\alpha}} ; \mathbb{Q}\right)\right)$, and the latter algebra is isomorphic to $U L\langle R\rangle$. Therefore, as $(\Omega W)_{*}$ is the multiplicative extension of $S_{*}$, it induces the multiplicative extension $U(i)$ of $\bar{i}$.

Finally, we bring $\mathcal{Z}_{K}(\underline{S})$ back into the picture.

Theorem 13.5. The map $\bigvee_{\alpha \in \mathcal{I}} S^{t_{\alpha}+1} \stackrel{W}{\longrightarrow} D J_{K}(\underline{S})$ lifts to $\mathcal{Z}_{K}(\underline{S})$, and induces a homotopy equivalence $\bigvee_{\alpha \in \mathcal{I}} S^{t_{\alpha}+1} \longrightarrow \mathcal{Z}_{K}(\underline{S})$.

Proof. By Lemma 10.2, each higher Whitehead product $w_{\sigma}$ lifts to $\mathcal{Z}_{K}(\underline{S})$. Therefore each iterated Whitehead product $\left[\left[w_{\sigma}, a_{j_{1}}\right], \ldots, a_{j_{l}}\right]$ into $D J_{K}(\underline{S})$ composes trivially to $\prod_{i=1}^{m} S^{n_{i}+1}$ and so lifts to $\mathcal{Z}_{K}(\underline{S})$. Hence there is a lift

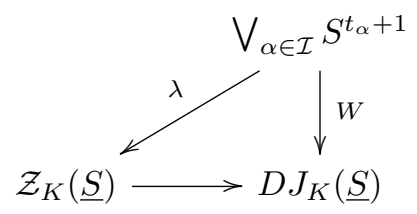

for some map $\lambda$.

After looping, Corollary 13.4 implies that the map $\Omega\left(\bigvee_{\alpha \in \mathcal{I}} S^{t_{\alpha}+1}\right) \stackrel{\Omega \lambda}{\longrightarrow} \Omega \mathcal{Z}_{K}(\underline{S})$ induces an inclusion $U L\langle R\rangle \stackrel{(\Omega \lambda)_{*}}{\longrightarrow} H_{*}\left(\Omega \mathcal{Z}_{K}(\underline{S}) ; \mathbb{Q}\right)$. By Proposition 13.3, there is an isomorphism $H_{*}\left(\Omega \mathcal{Z}_{K}(\underline{S}) ; \mathbb{Q}\right) \cong$ $U L\langle R\rangle$. Therefore a counting argument implies that the inclusion $(\Omega \lambda)_{*}$ must be an isomorphism. Hence $\Omega \lambda$ is a rational homotopy equivalence. That is, $\Omega \lambda$ induces an isomorphism of rational homotopy groups. Therefore, so does $\lambda$, and so $\lambda$ is a rational homotopy equivalence.

To upgrade this to an integral homotopy equivalence, proceed as follows.

Step 1: Let $g_{\sigma}: S^{N_{\sigma}+1} \longrightarrow \mathcal{Z}_{K}(\underline{S})$ be a lift of the higher Whitehead product $w_{\sigma}$. By Lemma 10.2, $g_{\sigma}$ can be chosen so that it has a left homotopy inverse. Now take the adjoint of (26) to obtain a homotopy commutative diagram

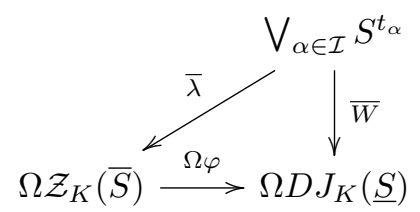

where $\bar{W}, \bar{\lambda}$ are the adjoints of $W, \lambda$ respectively. Let $\bar{g}_{\sigma}: S^{N_{\sigma}} \longrightarrow \Omega \mathcal{Z}_{K}(\underline{S})$ be the adjoint of $g_{\sigma}$. Then $\bar{g}_{\sigma}$ is homotopic to the composite $S^{N_{\sigma}} \stackrel{E}{\longrightarrow} \Omega S^{N_{\sigma}+1} \stackrel{\Omega g_{\sigma}}{\longrightarrow} \Omega \mathcal{Z}_{K}(\underline{S})$ where $E$ is the suspension map. Since $g_{\sigma}$ has a left homotopy inverse, so does $\Omega g_{\sigma}$. Since $E_{*}$ is the inclusion of the generator in $H_{*}\left(\Omega S^{N_{\sigma}+1} ; \mathbb{Z}\right) \cong \mathbb{Z}\left[x_{N_{\sigma}}\right]$, we have that $\bar{g}_{\sigma}$ has nonzero Hurewicz image $\bar{u}_{\sigma}$ for some element $\bar{u}_{\sigma} \in H_{t}\left(\Omega \mathcal{Z}_{K}(\underline{S}) ; \mathbb{Z}\right)$. Let $\bar{w}_{\sigma}: S^{N_{\sigma}} \longrightarrow \Omega D J_{K}(\underline{S})$ be the adjoint of $w_{\sigma}$. Since $g_{\sigma}$ is a lift of $w_{\sigma}$ through $\varphi, \bar{g}_{\sigma}$ is a lift of $\bar{w}_{\sigma}$ through $\Omega \varphi$. Therefore, if we identify $\bar{u}_{\sigma} \in H_{*}\left(\Omega \mathcal{Z}_{K}(\underline{S}) ; \mathbb{Z}\right)$ with its image in $H_{*}\left(\Omega D J_{K}(\underline{S}) ; \mathbb{Z}\right)$, then $\bar{w}_{\sigma}$ has Hurewicz image $\bar{u}_{\sigma}$. 
Step 2: Observe that the homotopy fibration $\Omega \mathcal{Z}_{K}(\underline{S}) \stackrel{\Omega \varphi}{\longrightarrow} \Omega D J_{K}(\underline{S}) \longrightarrow \prod_{i=1}^{m} S^{1}$ splits as

$$
\Omega D J_{K}(\underline{S}) \simeq \prod_{i=1}^{m} S^{1} \times \Omega \mathcal{Z}_{K}(\underline{S}) .
$$

For $1 \leq i \leq m$, the coordinate inclusion $S^{2} \stackrel{a_{i}}{\longrightarrow} D J_{K}(\underline{S})$ adjoints to a map $S^{1} \stackrel{\bar{a}_{i}}{\longrightarrow} \Omega D J_{K}(\underline{S})$. The decomposition of $\Omega D J_{K}(\underline{S})$ implies that $\bar{a}_{i}$ has a nonzero Hurewicz image $\bar{b}_{i} \in H_{1}\left(\Omega D J_{K}(\underline{S}) ; \mathbb{Z}\right)$. As Samelson products commute with Hurewicz images, the iterated Samelson product $\left[\left[\bar{w}_{\sigma}, \bar{a}_{j_{1}}\right], \ldots, \bar{a}_{j_{l}}\right]$ has Hurewicz image $\left[\left[\bar{u}_{\sigma}, \bar{b}_{j_{1}}\right], \ldots, \bar{b}_{j_{l}}\right]$.

Step 3: Now consider the rationalisation map

$$
r: H_{*}\left(\Omega D J_{K}(\underline{S}) ; \mathbb{Z}\right) \longrightarrow H_{*}\left(\Omega D J_{K}(\underline{S}) ; \mathbb{Q}\right) .
$$

The integral splittings of spheres off $\mathcal{Z}_{K}(\underline{S})$ via the maps $g_{\sigma}$ and the integral decomposition of $\Omega D J_{K}(\underline{S})$ induce corresponding rational splittings and a rational decomposition. Thus $r\left(\bar{u}_{\sigma}\right)=u_{\sigma}$ and $r\left(\bar{b}_{i}\right)=b_{i}$, where $u_{\sigma}$ and $b_{i}$ is the notation used for the rational classes in $H_{*}\left(\Omega D J_{K}(\underline{S}) ; \mathbb{Q}\right)$ in Corollary 6.4. As $r$ commutes with commutators, we therefore have $r\left(\left[\left[\bar{u}_{\sigma}, \bar{b}_{j_{1}}\right], \ldots, \bar{b}_{j_{l}}\right]\right)=$ $\left[\left[u_{\sigma}, b_{j_{1}}\right], \ldots, b_{j_{l}}\right]$. In particular, since $\left[\left[u_{\sigma}, b_{j_{1}}\right], \ldots, b_{j_{l}}\right]$ is a generator of $R$ (notation as in Corollary 6.4), the Hurewicz image $\left[\left[\bar{u}_{\sigma}, \bar{b}_{j_{1}}\right], \ldots, \bar{b}_{j_{l}}\right]$ in $H_{*}\left(\Omega D J_{K}(\underline{S}) ; \mathbb{Z}\right)$ is nonzero and maps by a degree one map to $\left[\left[u_{\sigma}, b_{j_{1}}\right], \ldots b_{j_{l}}\right]$.

Notice that the iterated Samelson product $\left[\left[\bar{w}_{\sigma}, \bar{a}_{j_{1}}\right], \ldots, \bar{a}_{j_{l}}\right]$ is the adjoint of the iterated Whitehead product $\left[\left[w_{\sigma}, a_{j_{1}}\right], \ldots, a_{j_{l}}\right]$. Therefore the collection of adjointed higher Whitehead products $\bar{w}_{\sigma}$ and iterated Samelson products $\left[\left[\bar{w}_{\sigma}, \bar{a}_{j_{1}}\right], \ldots, \bar{a}_{j_{l}}\right]$ comprise the map $\bar{W}$, and collectively lift by the map $\bar{\lambda}$ to $\Omega \mathcal{Z}_{K}(\underline{S})$. Each has an integral Hurewicz image which the rationalisation map $r$ sends by a degree one map to a generator in $R \subseteq H_{*}\left(\Omega \mathcal{Z}_{K}(\underline{S}) ; \mathbb{Q}\right) \subseteq H_{*}\left(\Omega D J_{K}(\underline{S}) ; \mathbb{Q}\right)$. Moreover, these integral Hurewicz images are linearly independent since their rationalizations are, and they are in one-to-one correspondence with the rational classes in $R$.

Step 4: By Theorem 1.4, $\mathcal{Z}_{K}(\underline{S})$ is homotopy equivalent to a wedge of simply-connected spheres, say $\mathcal{Z}_{K}(\underline{S}) \simeq \Sigma\left(\bigvee_{\beta \in \mathcal{J}} S^{t_{\beta}}\right)$. Thus $\langle R\rangle \cong H_{*}\left(\bigvee_{\beta \in \mathcal{J}} S^{t_{\beta}} ; \mathbb{Q}\right)$ and the inclusion of $R$ into $H_{*}\left(\Omega \mathcal{Z}_{K}(\underline{S}) ; \mathbb{Q}\right)$ is induced by the suspension map $E$. Therefore, $\bar{\lambda}$ maps $H_{*}\left(\bigvee_{\alpha \in \mathcal{I}} S^{t_{\alpha}} ; \mathbb{Z}\right)$ isomorphically onto a set $R^{\prime} \subseteq H_{*}\left(\bigvee_{\beta \in \mathcal{J}} S^{t_{\beta}} ; \mathbb{Z}\right)$, which the rationalization $r$ sends by degree one maps to $R$. But $r$ sends $H_{*}\left(\bigvee_{\beta \in \mathcal{J}} S^{t_{\beta}} ; \mathbb{Z}\right)$ by degree one maps to $H_{*}\left(\bigvee_{\beta \in \mathcal{J}} S^{t_{\beta}} ; \mathbb{Q}\right)$. Thus $\left\langle R^{\prime}\right\rangle \cong H_{*}\left(\bigvee_{\beta \in \mathcal{J}} S^{t_{\beta}} ; \mathbb{Z}\right)$. Adjointing, we obtain that the map $\bigvee_{\alpha \in \mathcal{I}} S^{t_{\alpha}+1} \stackrel{\lambda}{\longrightarrow} \mathcal{Z}_{K}(\underline{S})$ induces an isomorphism in integral homology. Hence $\lambda$ is an integral homotopy equivalence.

Proof of Theorem 8.7. This is simply a rephrasing of Theorem 13.5. 


\section{Properties of $\Omega D J_{K}$ AND $\Omega \mathcal{Z}_{K}$ FOR DIRECTED $M F$-COMPLEXES}

Recall that $\imath: S^{2} \longrightarrow \mathbb{C} P^{\infty}$ is the inclusion of the bottom cell. By naturality, there is a homotopy fibration diagram

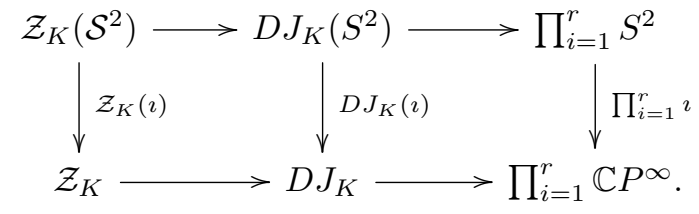

In this section we will use the calculations of $H_{*}\left(\Omega D J_{K}\left(S^{2}\right) ; \mathbb{Q}\right)$ and $H_{*}\left(\Omega \mathcal{Z}_{K}\left(\mathcal{S}^{2}\right) ; \mathbb{Q}\right)$ in Section 13 to calculate $H_{*}\left(\Omega D J_{K} ; \mathbb{Q}\right)$, proving Theorem 8.6 , and $H_{*}\left(\Omega \mathcal{Z}_{K} ; \mathbb{Q}\right)$. We then give a homotopy decomposition of $\mathcal{Z}_{K}$ as a wedge of spheres and describe the map $\mathcal{Z}_{K} \longrightarrow D J_{K}$ in terms of higher Whitehead products and iterated Whitehead products, proving Theorem 8.8.

By Proposition 11.2, $H_{*}\left(\Omega D J_{K}\right) \cong \operatorname{colim}_{\sigma \in M F(K)} H_{*}(\Omega F W(\sigma) ; \mathbb{Q})$, so we first need to calculate $H_{*}(\Omega F W(\sigma) ; \mathbb{Q})$ and then take a colimit to put the pieces together. We do this in Lemma 14.3 and Theorem 14.4 after two preliminary lemmas. In general, let $X_{1}, \ldots, X_{m}$ be path-connected pointed $C W$-complexes and consider the fat wedge $F W(\underline{X})$ in $\prod_{i=1}^{m} X_{i}$. Let $j$ be the inclusion $j: F W(\underline{X}) \longrightarrow \prod_{i=1}^{m} X_{i}$

Lemma 14.1. The map $\Omega F W(\underline{X}) \stackrel{\Omega j}{\longrightarrow} \prod_{i=1}^{m} \Omega X_{i}$ has a right homotopy inverse, which can be chosen to be natural for maps $X_{i} \longrightarrow Y_{i}$.

Proof. The inclusion $\bigvee_{i=1}^{m} X_{i} \longrightarrow F W(\underline{X})$ is natural, as are the inclusions $X_{i} \longrightarrow \bigvee_{i=1}^{m} X_{i}$ for $1 \leq i \leq m$. Now loop and consider the composite

$$
\bar{\mu}: \prod_{i=1}^{m} \Omega X_{i} \longrightarrow \prod_{i=1}^{m} \Omega\left(\bigvee_{i=1}^{m} X_{i}\right) \stackrel{\mu}{\longrightarrow} \Omega\left(\bigvee_{i=1}^{m} X_{i}\right) \longrightarrow \Omega F W(\underline{X}),
$$

where $\mu$ is the loop multiplication. All three maps in the composite are natural, and $\bar{\mu}$ is a right homotopy inverse of $\Omega j$.

Let $F$ be the homotopy fibre of $j$. As mentioned earlier, Porter [Po2] showed that there is a homotopy equivalence $F \simeq \Sigma^{m-1} \Omega X_{1} \wedge \cdots \wedge \Omega X_{m}$. Further, in [Po1, 1.2] he showed that this homotopy equivalence is natural for maps $X_{i} \longrightarrow Y_{i}$. We record this as follows.

Lemma 14.2. Let $f_{i}: X_{i} \longrightarrow Y_{i}$ be maps between simply-connected spaces. There is a homotopy commutative diagram between fibrations

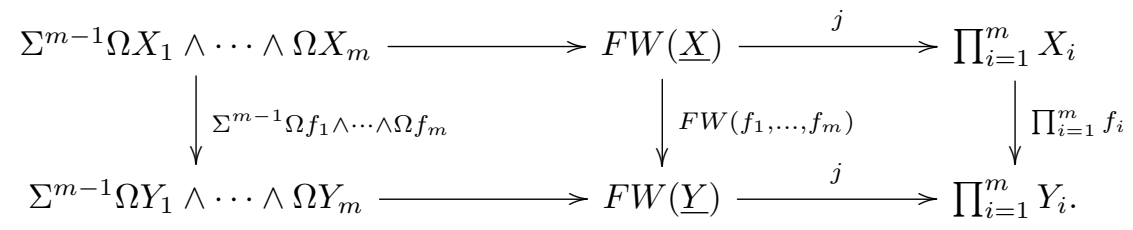


Let $F W\left(S^{2}\right)$ and $F W\left(\mathbb{C} P^{\infty}\right)$ be the fat wedges of $\prod_{i=1}^{m} S^{2}$ and $\prod_{i=1}^{m} \mathbb{C} P^{\infty}$ respectively. Let $F W(\imath): F W\left(S^{2}\right) \longrightarrow F W\left(\mathbb{C} P^{\infty}\right)$ be the map induced by the inclusion $S^{2} \stackrel{\imath}{\longrightarrow} \mathbb{C} P^{\infty}$.

Lemma 14.3. There is a commutative diagram of algebras

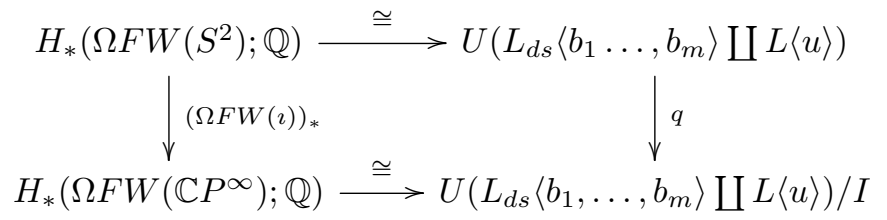

where $u$, of degree $2 m-2$, is the Hurewicz image of the adjoint of a higher Whitehead product, $I$ is the ideal $\left(b_{i}^{2},\left[u, b_{i}\right] \mid 1 \leq i \leq m\right)$, and $q$ is the quotient map.

Proof. The isomorphism for $H_{*}\left(\Omega F W\left(S^{2}\right) ; \mathbb{Q}\right)$ holds by Theorem 12.4. To obtain the compatible isomorphism for $H_{*}\left(\Omega F W\left(\mathbb{C} P^{\infty}\right) ; \mathbb{Q}\right)$ we first consider what happens on the level of spaces. For a space $X$, let $X^{(m)}$ be the $m$-fold smash product of $X$ with itself. By Lemma 14.2, the map $\imath$ induces a homotopy commutative diagram

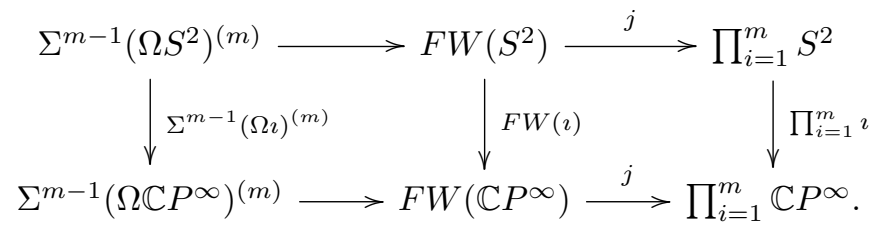

Note that $\Omega \mathbb{C} P^{\infty} \simeq S^{1}$ so $\Sigma^{m-1}\left(\Omega \mathbb{C} P^{\infty}\right)^{(m)} \simeq S^{2 m-1}$. Also, since $S^{1} \stackrel{E}{\longrightarrow} \Omega S^{2}$ is a right homotopy inverse for $\Omega$, if we let $s=\Sigma^{m-1} E^{(m)}$ and $t=\Sigma^{m-1}(\Omega i)^{(m)}$, then the composite $S^{2 m-1} \stackrel{s}{\longrightarrow}$ $\Sigma^{m-1}\left(\Omega S^{2}\right)^{(m)} \stackrel{t}{\longrightarrow} S^{2 m-1}$ is homotopic to the identity map.

After looping we obtain a homotopy commutative diagram

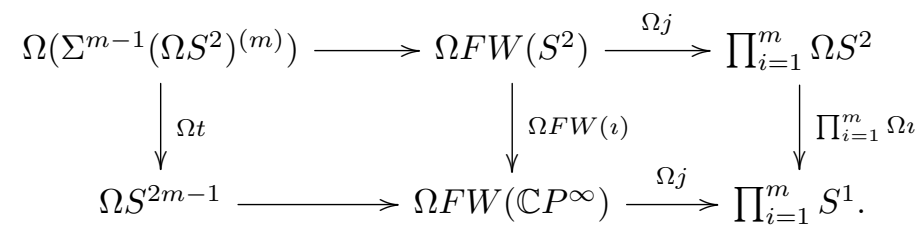

By Lemma 14.1, $\Omega j$ has a natural right homotopy inverse, so there is a homotopy commutative diagram of sections

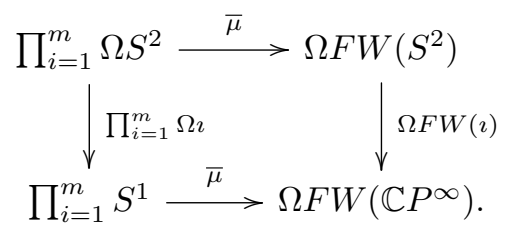

Now we examine the effect of (28) in homology. By Theorem 13.2 and Proposition 13.3, a model for the homology of the homotopy fibration along the top row of (27) is

$$
U L\langle R\rangle \stackrel{U(i)}{\longrightarrow} U\left(L_{d s}\left\langle b_{1}, \ldots, b_{m}\right\rangle \amalg L\langle u\rangle\right) \stackrel{U(\pi)}{\longrightarrow} U L_{d s}\left\langle b_{1}, \ldots, b_{m}\right\rangle
$$


where $R=\left\{\left[\left[u, b_{j_{1}}\right], \ldots, b_{j_{l}}\right] \mid 1 \leq j_{1} \leq \cdots \leq j_{l} \leq m, 0 \leq l<\infty\right\}$. From (28), we obtain a right inverse $\bar{\mu}_{*}$ of $U(\pi)$. In particular, if $H_{*}\left(\prod_{i=1}^{m} \Omega S^{2} ; \mathbb{Q}\right) \cong \mathbb{Q}\left[c_{1}, \ldots, c_{m}\right]$, then $\bar{\mu}_{*}\left(c_{i}\right)=b_{i}+\gamma_{i}$ for some $\gamma_{i} \in U L\langle R\rangle$. However, the least degree of $U L\langle R\rangle$ which is non-trivial is $2 m-2$, while $c_{i}$ has degree 1 . As $m \geq 3$, for degree reasons we must have $\gamma_{i}=0$. Thus $\bar{\mu}_{*}\left(c_{i}\right)=b_{i}$. For similar degree reasons, we have $\bar{\mu}_{*}\left(c_{i}^{2}\right)=b_{i}^{2}$ (even though $\bar{\mu}_{*}$ may not be multiplicative). On the other hand, $(\Omega \imath)_{*}$ is an isomorphism on the first homology group and the same is true after composing with $\bar{\mu}_{*}$, while $(\Omega \imath)_{*}\left(c_{i}^{2}\right)=0$. Thus the commutativity of (28) after taking homology implies that for $1 \leq i \leq m$, $(\Omega F W(\imath))_{*}$ is degree one on $b_{i}$ while $(\Omega F W(\imath))_{*}\left(b_{i}^{2}\right)=0$. The latter implies by multiplicativity that $(\Omega F W(\imath))_{*}$ sends the ideal $\left(b_{1}^{2}, \ldots, b_{m}^{2}\right)$ to 0 .

Next, consider the commutator $\left[u, b_{i}\right] \in H_{*}\left(\Omega F W\left(S^{2}\right) ; \mathbb{Q}\right)$. In terms of $(29),\left[u, b_{i}\right]$ composes trivially with $U(\pi)$ and so is the image of an element $\delta_{i} \in U L\langle R\rangle$. Note that $\delta_{i}$ has degree $2 n-1$. Taking homology in $(27)$, we see that $(\Omega t)_{*}\left(\delta_{i}\right)=0$ for degree reasons. Thus the commutativity of the left square in $(27)$ implies that $(\Omega F W(\imath))_{*}\left(\left[u, b_{i}\right]\right)=0$. By multiplicativity, $(\Omega F W(\imath))_{*}$ therefore sends the ideal $I=\left(b_{i}^{2},\left[u, b_{i}\right] \mid 1 \leq i \leq m\right)$ to 0.

Thus there is a factorization

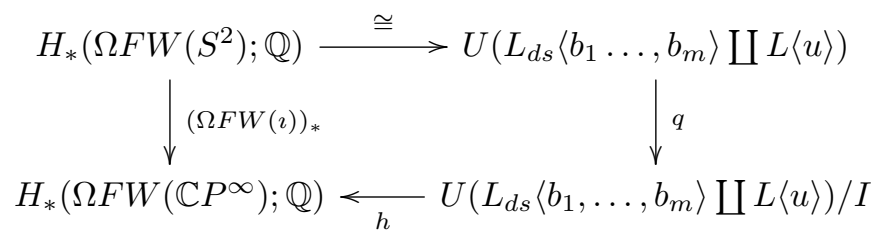

for some algebra map $h$, which is degree one on $b_{i}$ for each $1 \leq i \leq m$. In addition, the fact that $\Omega t$ has a right homotopy inverse implies that $h$ is degree one on $u$.

We claim that $h$ is an isomorphism, from which the lemma would follow. To see the isomorphism, let $I^{\prime}$ be the ideal $\left(\left[u, b_{i}\right] \mid 1 \leq i \leq m\right)$. Observe that $U\left(L_{d s}\left\langle b_{1}, \ldots, b_{m}\right\rangle \coprod L\langle u\rangle\right) / I^{\prime}$ is isomorphic to $U L_{d s}\left\langle b_{1}, \ldots, b_{m}, u\right\rangle \cong \mathbb{Q}\left[b_{1}, \ldots b_{m}, u\right]$. Thus $U\left(L_{d s}\left\langle b_{1}, \ldots, b_{m}\right\rangle \coprod L\langle u\rangle\right) / I$ is isomorphic to $\Lambda\left(b_{1}, \ldots, b_{m}\right) \otimes \mathbb{Q}[u]$. On the other hand, the section $\bar{\mu}$ in (28) implies that there is a homotopy decomposition $\Omega F W\left(\mathbb{C} P^{\infty}\right) \simeq\left(\prod_{i=1}^{m} S^{1}\right) \times \Omega S^{2 m-1}$. Thus there is a coalgebra isomorphism $H_{*}\left(\Omega F W\left(\mathbb{C} P^{\infty}\right) ; \mathbb{Q}\right) \cong \Lambda\left(c_{1}, \ldots, c_{m}\right) \otimes \mathbb{Q}[v]$ where $v$ has degree $2 m-2$. From the use of $\bar{\mu}$ and $t$ in both the homotopy decomposition of $\Omega F W\left(\mathbb{C} P^{\infty}\right)$ and the factorisation of $(\Omega F W(\imath))_{*}$ through $h$, we see that $h\left(b_{i}\right)=c_{i}$ for $1 \leq i \leq m$ and $h(u)=v$. As $h$ is an algebra map, it therefore induces an isomorphism.

Now we pass to a colimit of fat wedges to prove Theorem 8.6, restated below to match the simplifying condition in Remark 13.1.

Theorem 14.4. Let $K$ be a directed $M F$-complex such that $|\sigma|>2$ for every $\sigma \in M F(K)$. There is an algebra isomorphism

$$
H_{*}\left(\Omega D J_{K} ; \mathbb{Q}\right) \cong U\left(L_{d s}\left\langle b_{1}, \ldots, b_{m}\right\rangle \coprod L\left\langle u_{\sigma} \mid \sigma \in M F(K)\right\rangle\right) / I
$$


where $u_{\sigma}$ is the Hurewicz image of the adjoint of a higher Whitehead product and I is the ideal

$$
I=\left(b_{i}^{2},\left[u_{\sigma}, b_{j_{\sigma}}\right] \mid 1 \leq i \leq m, \sigma=\left(i_{1}, \ldots, i_{k}\right) \in M F(K), j_{\sigma} \in\left\{i_{1}, \ldots, i_{k}\right\}\right) .
$$

Further, there is a commutative diagram of algebras

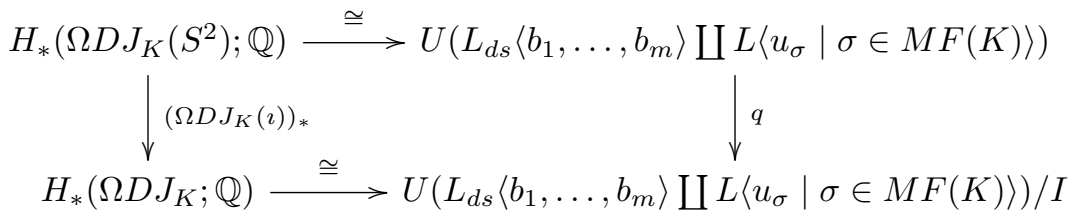

where $q$ is the quotient map.

Proof. With $\sigma=\left(i_{1}, \ldots, i_{k}\right)$, consider the diagram

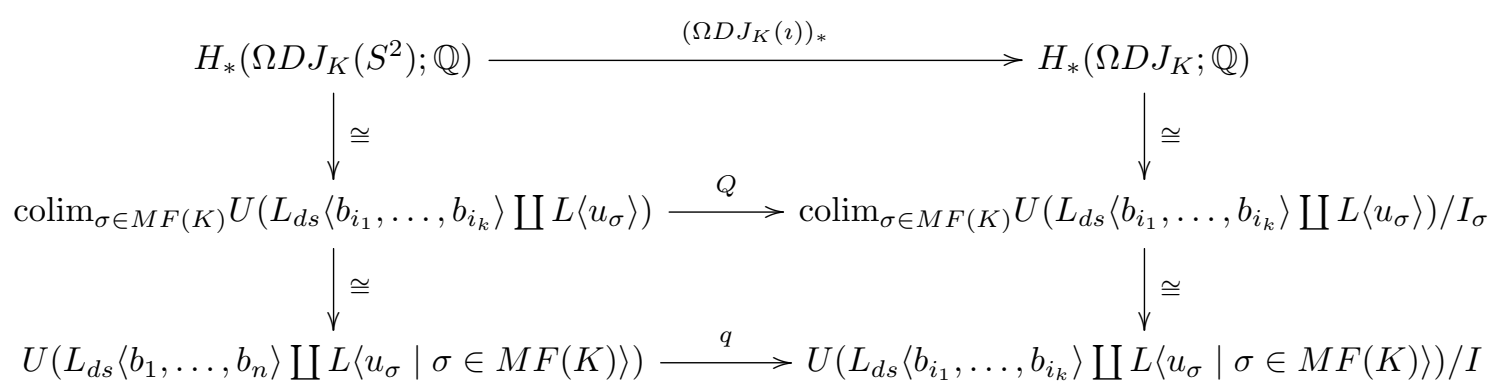

where $Q=\operatorname{colim}_{\sigma \in M F(K)} q_{\sigma}$ and $I_{\sigma}$ is the ideal generated by $\left(b_{\sigma_{i}}^{2},\left[u_{\sigma}, b_{j_{\sigma}}\right] \mid j_{\sigma} \in\left\{i_{1}, \ldots, i_{k}\right\}\right)$. The upper square commutes by combining Proposition 11.2 and Lemma 14.3. The lower square is the result of evaluating the colimit, and so commutes. Note that both squares commute as maps of algebras. The lower row is the epimorphism asserted by the theorem, and the outer rectangle is the asserted commutative diagram.

Next, we use Theorem 14.4 to calculate $H_{*}\left(\Omega \mathcal{Z}_{K} ; \mathbb{Q}\right)$ in Proposition 14.6 as the universal enveloping algebra of a certain free graded Lie algebra. This will involve some explicit calculations involving graded Lie algebra identities, which we recall now. In general, if $L$ is a graded Lie algebra over $\mathbb{Q}$ with bracket $[$,$] , there is a graded anti-symmetry identity [x, y]=-(-1)^{|x||y|}[y, x]$ for all $x, y \in L$ and a graded Jacobi identity $[[x, y], z]=[x,[y, z]]-(-1)^{|x||y|}[y,[x, z]]$ for all $x, y, z \in L$.

The ideal in Theorem 14.4 involves brackets of the form $\left[u_{\sigma}, b_{j}\right]$ where $j \in\left\{i_{1}, \ldots, i_{k}\right\}$, where $\sigma=\left(i_{1}, \ldots, i_{k}\right)$. Thus in the quotient we need to keep track of brackets of the form $\left[u_{\sigma}, b_{j}\right]$ where $j$ is in the complement of $\left\{i_{1}, \ldots, i_{k}\right\}$. Let $J_{\sigma}=\{1, \ldots, m\}-\left\{i_{1}, \ldots, i_{k}\right\}$. Consider the free graded Lie algebra generated by

$$
\widetilde{R}=\left\{\left[\left[u_{\sigma}, b_{j_{1}}\right], \ldots, b_{j_{l}}\right] \mid \sigma \in M F(K),\left\{j_{1}, \ldots, j_{l}\right\} \subseteq J_{\sigma}, 1 \leq j_{1}<\cdots<j_{l} \leq m, 0 \leq l \leq m\right\} .
$$

Note that each $j_{t}$ can appear at most once in any given bracket. This should be compared to the $\mathbb{Q}$-module $R$, where each $j_{t}$ can appear arbitrarily many times in a given bracket. Let $i_{R}: \widetilde{R} \longrightarrow R$ be the inclusion and $\pi_{R}: R \longrightarrow \widetilde{R}$ be the projection. 
Lemma 14.5. There is a short exact sequence of Lie algebras

$$
L\langle\widetilde{R}\rangle \stackrel{\tilde{i}}{\longrightarrow}\left(L_{d s}\left\langle b_{1}, \ldots, b_{m}\right\rangle \amalg L\left\langle u_{\sigma} \mid \sigma \in M F(K)\right\rangle\right) / \widetilde{I} \stackrel{\widetilde{\pi}}{\longrightarrow} L_{d s}\left\langle b_{1}, \ldots, b_{m}\right\rangle / \widetilde{I}^{\prime}
$$

where $\widetilde{I}$ is the ideal $\widetilde{I}=\left(\left[b_{i}, b_{i}\right],\left[u_{\sigma}, b_{j_{\sigma}}\right] \mid 1 \leq i \leq m, \sigma \in M F(K), j_{\sigma} \in\left\{i_{1}, \ldots, i_{k}\right\}\right), \widetilde{I}^{\prime}$ is the ideal $\left(\left[b_{i}, b_{i}\right] \mid 1 \leq i \leq m\right), \tilde{i}$ is the inclusion, and $\tilde{\pi}$ is the projection.

Proof. To simplify notation, let $L=L_{d s}\left\langle b_{1}, \ldots, b_{m}\right\rangle \coprod L\left\langle u_{\sigma} \mid \sigma \in M F(K)\right\rangle$. Observe from the definitions of $\widetilde{I}$ and $\widetilde{I}^{\prime}$ that there is a commutative diagram

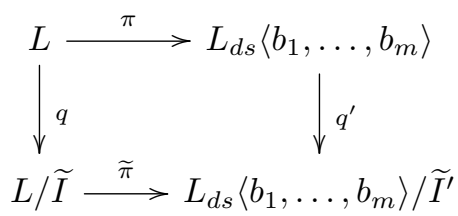

where $q$ and $q^{\prime}$ are the quotient maps. By (24), the kernel of $\pi$ is $L\langle R\rangle$. Let $\widetilde{L}$ be the kernel of $\widetilde{\pi}$. The commutativity of the diagram implies that there is an induced map $\tilde{q}: L\langle R\rangle \longrightarrow \widetilde{L}$.

We claim that $\tilde{q}$ is a surjection. Let $x \in \widetilde{L}$ and let $x$ also denote its image in $L / \widetilde{I}$. As $q$ is onto there is an element $y \in L$ such that $q(y)=x$. Let $z=\pi(y)$. If $z=0$ then by exactness $y$ lifts to $\tilde{y} \in L\langle R\rangle$ and so $\tilde{q}(\tilde{y})=x$. If $z \neq 0$, then $q^{\prime}(z)=0$ by exactness. Since $L$ is a coproduct, the projection $\pi$ has a right inverse $r: L_{d s}\left\langle b_{1}, \ldots, b_{m}\right\rangle \longrightarrow L$ which is a map of Lie algebras. As the generators of the ideal $\widetilde{I}^{\prime}$ are all generators of the ideal $\widetilde{I}$, we have $(q \circ r)(b)=0$ if and only if $q^{\prime}(b)=0$ for any $b \in L_{d s}\left\langle b_{1}, \ldots, b_{m}\right\rangle$. Thus $r(z)$ has the property that $(q \circ r)(z)=0$. Therefore $\tilde{y}=y-r(z)$ lifts to $L\langle R\rangle$ and $q(y-r(z))=q(y)=x$, so $\tilde{q}(\tilde{y})=x$. Hence $\tilde{q}$ is a surjection.

Now $\tilde{q}$ is a surjection and $\widetilde{L}$ injects into $L / \widetilde{I}$. Therefore $\widetilde{L}$ is isomorphic to the image of $L\langle R\rangle$ under $q$. We next show that this image is $L\langle\widetilde{R}\rangle$. We first perform two short calculations.

Calculation 1: The Jacobi identity states that $\left[\left[a, b_{i}\right], b_{j}\right]=\left[a,\left[b_{i}, b_{j}\right]\right]-(-1)^{|a|\left|b_{i}\right|}\left[b_{i},\left[a, b_{j}\right]\right]$ for any $a \in L$ and any $1 \leq i, j \leq m$. The abelian property of $L_{d s}\left\langle b_{1}, \ldots, b_{m}\right\rangle$ implies that $\left[b_{i}, b_{j}\right]=0$ and so $\left[a,\left[b_{i}, b_{j}\right]\right]=0$. By the anti-symmetry identity, $-(-1)^{|a|\left|b_{i}\right|}\left[b_{i},\left[a, b_{j}\right]\right]=(-1)^{|a|\left|b_{i}\right|+\left|b_{i}\right|\left|\left[a, b_{j}\right]\right|}\left[\left[a, b_{j}\right], b_{i}\right]$.

Since $\left|b_{i}\right|=1$ for $1 \leq i \leq m$, the sign on the right side of this equation equals $(-1)^{2|a|+1}$, which is -1 . Therefore $\left[\left[a, b_{i}\right], b_{j}\right]=-\left[\left[a, b_{j}\right], b_{i}\right]$.

Calculation 2: The Jacobi identity states that $\left[\left[a, b_{i}\right], b_{i}\right]=\left[a,\left[b_{i}, b_{i}\right]\right]-(-1)^{|a|\left|b_{i}\right|}\left[b_{i},\left[a, b_{i}\right]\right]$ for any $a \in$ $L$ and $1 \leq i \leq m$. Since $\left[b_{i}, b_{i}\right]=0$ in $L$ as $\left|b_{i}\right|$ is even, we have $\left[a,\left[b_{i}, b_{i}\right]\right]=0$. As in Calculation 1 , the anti-symmetry identity shows that $-(-1)^{\left|u_{\sigma}\right|}\left[b_{i},\left[a, b_{i}\right]\right]=-\left[\left[a, b_{i}\right], b_{i}\right]$. Thus $\left[\left[a, b_{i}\right], b_{i}\right]=-\left[\left[a, b_{i}\right], b_{i}\right]$, and so $2\left[\left[a, b_{i}\right], b_{i}\right]=0$. As $L$ is a Lie algebra over $\mathbb{Q}, 2$ is invertible and so $\left[\left[a, b_{i}\right], b_{i}\right]=0$.

By Calculation 1, up to sign change, whenever consecutive b's appear in a bracket of $L\langle R\rangle$ or $L$ their order can be interchanged. By Calculation 2, the effect of taking the quotient in $L\langle R\rangle$ and $L$ by the ideal $I^{\prime}=\left(\left[b_{i}, b_{i}\right] \mid 1 \leq i \leq n\right)$ is to annihilate all brackets in which appears a copy of $\left[a,\left[b_{i}, b_{i}\right]\right]$. Together with Calculation 1 which lets us freely interchange consecutive $b$ 's, any bracket 
of the form $\left[\left[u_{\sigma}, b_{j_{1}}\right], \ldots, b_{j_{l}}\right]$ is zero if any $b_{j_{t}}$ appears more than once. Thus, the only such nontrivial brackets must have $1 \leq j_{1}<\cdots<j_{l} \leq m, 0 \leq l<n$, as in the definition of $\widetilde{R}$. The effect of then taking the quotient by the ideal generated by $\left[u_{\sigma}, b_{j_{\sigma}}\right]$ for $j_{\sigma} \in\left\{i_{1}, \ldots, i_{k}\right\}$ is to annihilate those brackets in $\left\{\left[\left[u_{\sigma}, b_{j_{1}}\right], \ldots, b_{j_{l}}\right] \mid \sigma \in M F(K), 1 \leq j_{1}<\cdots<j_{l} \leq n, 0 \leq l<m\right\}$ which do not have $j_{1}, \ldots, j_{l} \in J_{\sigma}$. Thus the image of $L\langle R\rangle$ under $q$ is $L\langle\widetilde{R}\rangle$.

In general, the image of a graded Lie algebra $L$ in its universal enveloping algebra $U L$ has the property that $[x, y]=x y-(-1)^{|x||y|} y x$, where the multiplication is taking place in $U L$. In particular, the anti-symmetry identity implies that $[x, x]=2 x^{2}$ if the degree of $x$ is odd. Thus if 2 has been inverted in the ground ring, then the ideal in $U L$ generated by $[x, x]$ is identical to the ideal generated by $x^{2}$. In our case, the short exact sequence of Lie algebras in Lemma 14.5 implies that there is a short exact sequence of Hopf algebras

$$
U L\langle\widetilde{R}\rangle \longrightarrow U\left(L_{d s}\left\langle b_{1}, \ldots, b_{m}\right\rangle \amalg L\left\langle u_{\sigma} \mid \sigma \in M F(K)\right\rangle\right) / I \stackrel{\pi}{\longrightarrow} U L_{d s}\left\langle b_{1}, \ldots, b_{m}\right\rangle / I^{\prime}
$$

where $I$ is the ideal in Theorem 8.6 and $I^{\prime}=\left(b_{i}^{2} \mid 1 \leq i \leq m\right)$.

Proposition 14.6. There is a commutative diagram of algebras

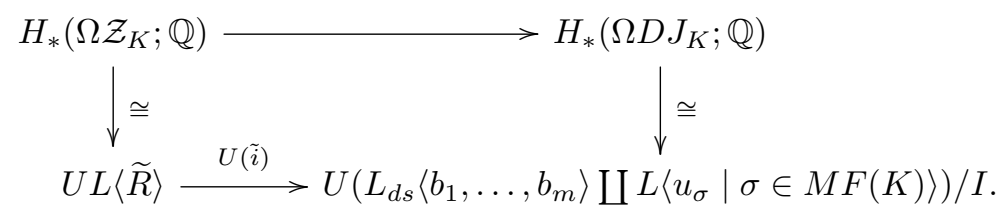

Proof. Argue as in Proposition 13.3, replacing the short exact sequence of Hopf algebras appearing there with that in (31), and replacing Theorem 13.2 with Theorem 14.4.

Now we use the description of $H_{*}\left(\Omega D J_{K} ; \mathbb{Q}\right)$ in Theorem 14.4 to produce maps as was done in the case of $\Omega D J_{K}(\underline{S})$. For $\sigma \in M F(K)$, let

$$
\tilde{w}_{\sigma}: S^{2|\sigma|-1} \stackrel{\phi_{k}}{\longrightarrow} F W\left(S^{2}, \sigma\right) \stackrel{F W(\imath)}{\longrightarrow} F W(\sigma) \longrightarrow D J_{K}
$$

be the higher Whitehead product. By Theorem 14.4, the element $u_{\sigma} \in H_{*}\left(\Omega D J_{K}(\underline{S}) ; \mathbb{Q}\right)$ is the Hurewicz image of the adjoint of $\tilde{w}_{\sigma}$. For $1 \leq i \leq m$, let $\tilde{a}_{i}$ be the composite

$$
\tilde{a}_{i}: S^{2} \stackrel{\imath}{\longrightarrow} \mathbb{C} P^{\infty} \longrightarrow D J_{K}
$$

where the right map is the $i^{t h}$-coordinate inclusion. Let $\widetilde{\mathcal{I}}$ be the index set for $\widetilde{R}$. Then $\widetilde{\alpha} \in \widetilde{\mathcal{I}}$ corresponds to a face $\sigma \in M F(K)$ and a sequence $\left(j_{1}, \ldots, j_{l}\right)$ where $1 \leq j_{1}<\cdots<j_{l} \leq m$ and $0 \leq l \leq m$. Given such an $\widetilde{\alpha}$, let $t_{\widetilde{\alpha}}=N_{\sigma}+l-2$. The inclusion $i_{R}$ induces a map $\widetilde{i}_{R}: \bigvee_{\widetilde{\alpha} \in \widetilde{\mathcal{I}}} S^{t_{\tilde{\alpha}}+1} \longrightarrow$ $\bigvee_{\alpha \in \mathcal{I}} S^{t_{\alpha}+1}$. Note that $\left(\widetilde{\Omega i}_{R}\right)_{*}$ can be identified with $U\left(i_{R}\right)$. Consider the composite

$$
\widetilde{W}: \bigvee_{\widetilde{\alpha} \in \widetilde{\mathcal{I}}} S^{t_{\tilde{\alpha}}+1} \stackrel{\widetilde{i}_{R}}{\longrightarrow} \bigvee_{\alpha \in \mathcal{I}} S^{t_{\alpha}+1} \stackrel{W}{\longrightarrow} D J_{K}\left(S^{2}\right) \stackrel{D J_{K}(\imath)}{\longrightarrow} D J_{K}
$$


If $\widetilde{\alpha}$ indexes $\sigma \in M F(K)$, then the restriction of $\widetilde{W}$ to $S^{t_{\tilde{\alpha}}+1}$ is the higher Whitehead product $\widetilde{w}_{\sigma}$. Otherwise, the restriction of $\widetilde{W}$ to $S^{t_{\tilde{\alpha}}+1}$ is an iterated Whitehead product of a single $\widetilde{w}_{\sigma}$ with some selection of the coordinate inclusions $\tilde{a}_{1}, \ldots, \tilde{a}_{m}$, where each $\tilde{a}_{i}$ appears at most once.

Corollary 14.7. The map $\Omega\left(\bigvee_{\widetilde{\alpha} \in \widetilde{\mathcal{I}}} S^{t \tilde{\alpha}+1}\right) \stackrel{\Omega \widetilde{W}}{\longrightarrow} \Omega D J_{K}$ induces in homology the map $U L\langle\widetilde{R}\rangle \stackrel{U(\tilde{i})}{\longrightarrow}$ $U\left(L_{d s}\left\langle b_{1}, \ldots, b_{m}\right\rangle \amalg L\left\langle u_{\sigma} \mid \sigma \in M F(K)\right\rangle\right) / I$.

Proof. Let $\widetilde{S}$ be the composite

$$
\widetilde{S}: \bigvee_{\widetilde{\alpha} \in \widetilde{\mathcal{I}}} S^{t_{\tilde{\alpha}+1}} \stackrel{E}{\longrightarrow} \Omega\left(\bigvee_{\widetilde{\alpha} \in \widetilde{\mathcal{I}}} S^{t_{\tilde{\alpha}+1}}\right) \stackrel{\Omega \widetilde{W}}{\longrightarrow} \Omega D J_{K} .
$$

The definition of $\widetilde{W}$ implies that $\widetilde{S}_{*}$ induces the composite

$$
\widetilde{R} \hookrightarrow U L\langle\widetilde{R}\rangle \stackrel{U L\left(i_{R}\right)}{\longrightarrow} U L\langle R\rangle \stackrel{(\Omega W)_{*}}{\longrightarrow} H_{*}\left(\Omega D J_{K}\left(S^{2}\right)\right) \stackrel{\left(\Omega D J_{K}(\imath)\right)_{*}}{\longrightarrow} H_{*}\left(\Omega D J_{K}\right) .
$$

Now argue as in Corollary 13.4, using the description of $\left(\Omega D J_{K}(\imath)\right)_{*}$ in Theorem 14.4, to obtain the asserted inclusion in homology.

We finish by bringing $\mathcal{Z}_{K}$ back into the picture.

Theorem 14.8. The map $\bigvee_{\widetilde{\alpha} \in \tilde{\mathcal{I}}} S^{S_{\tilde{\alpha}+1}} \stackrel{\widetilde{W}}{\longrightarrow} D J_{K}$ lifts to $\mathcal{Z}_{K}$, and induces a homotopy equivalence $\bigvee_{\widetilde{\alpha} \in \widetilde{\mathcal{I}}} S^{t_{\tilde{\alpha}}+1} \longrightarrow \mathcal{Z}_{K}$

Proof. Argue as in Theorem 13.5, using Proposition 14.6 and Corollary 14.7 in place of Proposition 13.3 and Corollary 13.4 respectively.

Proof of Theorem 8.8: This is simply a rephrasing of Theorem 14.8.

\section{Examples}

First, we consider the example of the shifted complex (19) which appeared in Section 8.

Example 15.1. Let $K$ be the following simplicial complex on 4 vertices

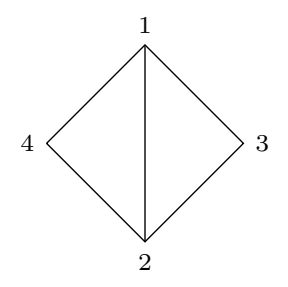

Under this ordering of the vertices, $K$ is shifted. The missing faces of $K$ are given by $M F(K)=$ $\{(3,4),(1,2,3),(1,2,4)\}$. Observe that in this case $|K|=\bigcup_{\sigma \in M F(K)}|\partial \sigma|$. In fact, $K=\partial(1,2,3) \cup$ $\partial(1,2,4)$, where the boundaries of the two missing faces have been glued along the common face $(1,2)$. So $K$ is a directed $M F$-complex. 
By Theorem 8.5, there is an algebra isomorphism

$$
H_{*}\left(\Omega D J_{K}(\underline{S})\right) \cong U\left(L_{d s}\left\langle b_{1}, b_{2}, b_{3}, b_{4}\right\rangle \amalg L\left\langle u_{1}, u_{2}, u_{3}\right\rangle\right) / J
$$

where $u_{1}$ is the Hurewicz image of the adjoint of the Whitehead product corresponding to the missing face $(3,4)$, and $u_{2}, u_{3}$ are the Hurewicz images of the adjoints of the higher Whitehead products corresponding to the missing faces $(1,2,3),(1,2,4)$, respectively. The ideal $J$ is determined by Jacobi identities and face relations based on the one missing face $(3,4)$ of dimension 1 . Specifically, observe that the Jacobi identity gives $\left[u_{1}, b_{1}\right]=\left[\left[b_{3}, b_{4}\right], b_{1}\right]=\left[b_{3},\left[b_{4}, b_{1}\right]\right]+\left[b_{4},\left[b_{3}, b_{1}\right]\right]$. As both $(1,3)$ and $(1,4)$ are faces of $K$, we have $\left[b_{3}, b_{1}\right]=0$ and $\left[b_{4}, b_{1}\right]=0$. Therefore $\left[u_{1}, b_{1}\right]=0$. Similarly, $\left[u_{1}, b_{2}\right]=0$. Thus $J=\left(\left[u_{1}, b_{1}\right],\left[u_{1}, b_{2}\right]\right)$.

By Theorem 8.7, the wedge summands of $\mathcal{Z}_{K}(\underline{S})$ and the maps to $D J_{K}(\underline{S})$ are as follows. For simplicity, we assume that each of the spheres in $\underline{S}$ is $S^{2}$. Part (a) gives the three summands $S^{3}$, $S^{5}$ and $S^{5}$ with maps $w_{1}, w_{2}$ and $w_{3}$ respectively. Since the missing faces $(1,2,3)$ and $(1,2,4)$ are of dimension greater than 1, part (b) gives the following additional summands and maps:

$$
\begin{aligned}
& {\left[\left[w_{2}, a_{j_{1}}\right], \ldots, a_{j_{l}}\right]: S^{5+l} \longrightarrow D J_{K}(\underline{S})} \\
& {\left[\left[w_{3}, a_{j_{1}}\right], \ldots, a_{j_{l}}\right]: S^{5+l} \longrightarrow D J_{K}(\underline{S})}
\end{aligned}
$$

for each list $1 \leq j_{1} \leq \cdots \leq j_{l} \leq l$ with $1 \leq l<\infty$. Note that the collection of spheres in (32) is identical to the collection in (33). In either case, let $W_{2}$ be the wedge of spheres. Since the missing face $(3,4)$ is of dimension 1 , part (c) gives the following additional summands:

$$
\left[\left[w_{1}, a_{j_{1}}\right], \ldots, a_{j_{l}}\right]: S^{3+l} \longrightarrow D J_{K}(\underline{S})
$$

for each list $1 \leq j_{1} \leq \cdots \leq j_{l} \leq l$ with $1 \leq l<\infty$ and each $j_{i} \in\{3,4\}$. Note that the restriction to $j_{i} \in\{3,4\}$ is from the fact that the Whitehead products $\left[w_{1}, a_{1}\right],\left[w_{1}, a_{2}\right]$ correspond to elements in the ideal $J$, so any iterated Whitehead product involving $a_{1}$ or $a_{2}$ must be excluded from the list of independent Whitehead products $W_{(3,4)}$. Let $W_{1}$ be the wedge of all possible spheres in (34).

Collectively, we obtain a homotopy equivalence

$$
\mathcal{Z}_{K}(\underline{S}) \simeq S^{3} \vee 2 S^{5} \vee W_{1} \vee 2 W_{2}
$$

and a map

$$
\mathcal{Z}_{K}(\underline{S}) \simeq S^{3} \vee 2 S^{5} \vee W_{1} \vee 2 W_{2} \longrightarrow D J_{K}(\underline{S})
$$

which is the wedge sum of $w_{1}, w_{2}, w_{3}$ and the three lists of iterated Whitehead products in (34), (32) and (33).

It is useful to reorganize the wedge summands of $\mathcal{Z}_{K}(\underline{S})$. Recall that the join of two spaces $A$ and $B$ is denoted $A * B$; for our purposes it suffices to know that $A * B \simeq \Sigma A \wedge B$. The right half-smash of $A$ and $B$ is the quotient space $A \rtimes B=(A \times B) /(* \times B)$. If $A$ is a suspension, then 
$A \rtimes B \simeq A \vee(A \wedge B)$. For $1 \leq i \leq 4$, let $S_{i}^{2}$ be the copy of $S^{2}$ associated with vertex $i$. Observe that the wedge summands of $S^{3} \vee W_{1}$ are in one-to-one correspondence with the wedge summands of $\Omega S_{3}^{2} * \Omega S_{4}^{2}$, where the James splitting is used to iteratively decompose this space into a wedge of spheres. As well, the wedge summands of $S^{5} \vee W_{2}$ from (32) are in one-to-one correspondence with the wedge summands of $\left(\Omega S_{1}^{2} * \Omega S_{2}^{2} * \Omega S_{3}^{3}\right) \rtimes \Omega S^{4}$, and the wedge summands of $S^{5} \vee W_{2}$ from (33) are in one-to-one correspondence with the wedge summands of $\left(\Omega S_{1}^{2} * \Omega S_{2}^{2} * \Omega S_{4}^{3}\right) \rtimes \Omega S^{3}$. Thus the wedge decomposition of $\mathcal{Z}_{K}(\underline{S})$ above agrees with that in [GT2, Section 6].

Next, by Theorem 8.6, there is an algebra isomorphism

$$
H_{*}\left(\Omega D J_{K}\right) \cong U\left(L_{d s}\left\langle b_{1}, b_{2}, b_{3}, b_{4}\right\rangle \coprod L\left\langle u_{1}, u_{2}, u_{3}\right\rangle\right) /(I+J)
$$

where $u_{1}$ is the Hurewicz image of the adjoint of the Whitehead product $\widetilde{w}_{1}: S^{3} \longrightarrow \mathbb{C} P_{3}^{\infty} \vee \mathbb{C} P_{4}^{\infty} \longrightarrow$ $D J_{K}$, while $u_{2}$ and $u_{3}$ are the Hurewicz images of the adjoints of the higher Whitehead products $\widetilde{w}_{2}: S^{5} \longrightarrow F W(1,2,3) \longrightarrow D J_{K}$, and $\widetilde{w}_{3}: S^{5} \longrightarrow F W(1,2,4) \longrightarrow D J_{K}$, respectively; $J$ is as above, and $I=\left(b_{i}^{2},\left[u_{1}, b_{3}\right],\left[u_{1}, b_{4}\right],\left[u_{2}, b_{1}\right],\left[u_{2}, b_{2}\right],\left[u_{2}, b_{3}\right],\left[u_{3}, b_{1}\right],\left[u_{3}, b_{2}\right],\left[u_{3}, b_{4}\right]\right)$.

By Theorem 8.8, the wedge summands of $\mathcal{Z}_{K}$ and the maps to $D J_{K}$ are as follows. Part (a) gives the three summands $S^{3}, S^{5}$ and $S^{5}$ with maps $\widetilde{w}_{1}, \widetilde{w}_{2}$ and $\widetilde{w}_{3}$ respectively. Since the missing faces $(1,2,3)$ and $(1,2,4)$ are of dimension greater than 1 , part (b) gives two additional summands $S^{6}$ and $S^{6}$ from the iterated Whitehead products

$$
\left[\widetilde{w}_{2}, \tilde{a}_{4}\right]: S^{6} \longrightarrow D J_{K} \text { and }\left[\widetilde{w}_{3}, \tilde{a}_{3}\right]: S^{6} \longrightarrow D J_{K}
$$

For the missing face $(3,4)$ of dimension 1 , part (c) is vacuous in this case. To see this, observe that the Whitehead products $\left[\widetilde{w}_{1}, \tilde{a}_{3}\right]$ and $\left[\widetilde{w}_{1}, \tilde{a}_{4}\right]$ correspond to the algebraic elements $\left[u_{1}, a_{3}\right]$ and $\left[u_{1}, a_{4}\right]$, both of which appear in the ideal $I$, while the Whitehead products $\left[\widetilde{w}_{1}, \tilde{a}_{1}\right]$ and $\left[\widetilde{w}_{1}, a_{2}\right]$ correspond to the algebraic elements $\left[u_{1}, b_{1}\right]$ and $\left[u_{1}, b_{2}\right]$, both of which appear in the ideal $J$. Thus the collection of independent Whitehead products $\widetilde{W}_{(3,4)}$ is empty.

Collectively then, we obtain a homotopy equivalence $\mathcal{Z}_{K} \simeq S^{3} \vee 2 S^{5} \vee 2 S^{6}$ and a map

$$
S^{3} \vee 2 S^{5} \vee 2 S^{6} \longrightarrow D J_{K}
$$

which is the wedge sum of $\widetilde{w}_{1}, \widetilde{w}_{2}, \widetilde{w}_{3},\left[\widetilde{w}_{2}, \tilde{a}_{4}\right]$ and $\left[\widetilde{w}_{3}, \tilde{a}_{3}\right]$. Note that the homotopy equivalence matches that of [GT1, Example 10.2], which was calculated by different means.

The next example is similar to the previous one, but boosted up one dimension. 
Example 15.2. Let $K$ be the following simplicial complex on 5 vertices

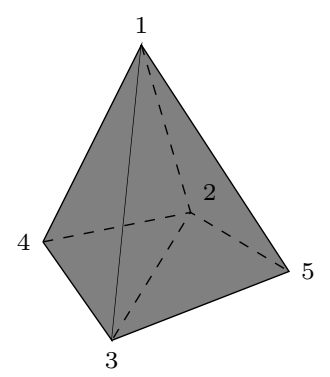

Here, $K=\partial(1,2,3,4) \cup \partial(1,2,3,5)$ where $\partial(1,2,3,4)$ and $\partial(1,2,3,5)$ have been glued along the common face $(1,2,3)$. This implies that $K$ is a directed $M F$-complex. Also, under this ordering of the vertices, $K$ is shifted. The missing faces of $K$ are given by $M F(K)=\{(4,5),(1,2,3,4),(1,2,3,5)\}$. This example is analogous to the previous one, so the algebraic descriptions of $H_{*}\left(\Omega D J_{K}(\underline{S})\right)$ and $H_{*}\left(\Omega D J_{K}\right)$ are as before, but with a dimensional shift to account for the fact that the higher Whitehead products corresponding to the missing faces $(1,2,3,4)$ and $(1,2,3,5)$ are maps $w_{i}: S^{7} \longrightarrow$ $D J_{K}(\underline{S})$ and $\widetilde{w}_{i}: S^{7} \longrightarrow D J_{K}$ for $i \in\{2,3\}$. In particular, arguing as before, we obtain a homotopy equivalence $\mathcal{Z}_{K} \simeq S^{3} \vee 2 S^{7} \vee 2 S^{8}$ and a map

$$
S^{3} \vee 2 S^{7} \vee 2 S^{8} \longrightarrow D J_{K}
$$

which is the wedge sum of $\widetilde{w}_{1}, \widetilde{w}_{2}, \widetilde{w}_{3},\left[\widetilde{w}_{2}, \tilde{a}_{5}\right]$ and $\left[\widetilde{w}_{3}, \widetilde{a}_{4}\right]$.

\section{REFERENCES}

[AH] J.F. Adams and P.J. Hilton, On the chain algebra of a loop space, Comment. Math. Helv. 30 (1955), 305-330.

[Al] C. Allday, Rational Whitehead products and a spectral sequence of Quillen, II, Houston J. Math. 3 (1977), 301-308.

[An] D.J. Anick, Hopf algebras up to homotopy, J. Amer. Math. Soc. 2 (1989), 417-453.

[BBCG1] A. Bahri, M. Bendersky, F.R. Cohen, and S. Gitler, The polyhedral product functor: a method of decomposition for moment-angle complexes, arrangements and related spaces, Adv. Math. 225 (2010), 1634-1668.

[BBCG2] A. Bahri, M. Bendersky, F.R. Cohen and S. Gitler, Cup-products for the polyhedral product functor, Math. Proc. Camb. Phil. Soc. 153 (2012), 457-469.

[BBCG3] A. Bahri, M. Bendersky, F.R. Cohen and S. Gitler, On the rational homotopy type of moment-angle complexes, arXiv:1206.2173.

[BBCG4] A. Bahri, M. Bendersky, F.R. Cohen and S. Gitler, Operations on polyhedral products and a new construction of infinite families of toric manifolds, arXiv:1011.0094.

[BBP] I.V. Baskakov, V.M. Buchstaber and T.E. Panov, Cellular cochain algebras and torus action, Uspekhi Mat. Nauk 59 (2004), 159-160 (Russian); Russian Math. Surveys 59 (2004), 562-563 (English translation).

[BG] P. Beben and J. Grbić, Configuration spaces and polyhedral products, arXiv:1409.4462.

[BJ] A. Berglund and M. Jöllenbeck, On the Golod property of Stanley-Reisner rings, J. Algebra 315 (2007), 246-273. 
[BW] A. Björner and M.I. Wachs, Nonpure shellable complexes and posets I, Trans. Amer. Math. Soc. 348 (1996), 1299-1327.

[BWW] A. Björner, M. Wachs and V. Welker, On sequentially Cohen-Macaulay complexes and posets, Israel J. Math. 169 (2009), 295-316.

[BM] F. Bosio and L. Meersseman, Real quadrics in $\mathbb{C}^{n}$, complex manifolds and convex polytopes, Acta Math. 197 (2006), 53-127.

[B] P. Bubenik, Separated Lie models and the homotopy Lie algebra, J. Pure Appl. Algebra 212 (2008), 401-410.

[BP1] V.M. Buchstaber and T.E. Panov, Torus actions, combinatorial topology and homological algebra, Uspekhi Mat. Naut. 55 (2000), 3-106 (Russian); Russian Math. Surveys 55 (2000), 825-921 (English translation).

[BP2] V.M. Buchstaber and T.E. Panov, Torus actions and their applications in topology and combinatorics, University Lecture Series 24, American Mathematical Society, 2002.

[CMN] F.R. Cohen, J.C. Moore, and J.A. Neisendorfer, Torsion in homotopy groups, Ann. of Math. 109 (1979), 121-168.

[CMS] S. Choi, M. Masuda and D.Y. Suh, Rigidity problems in toric topology, a survey, Proc. Steklov Inst. Math. 275 (2011), 177-190.

[DJ] M.W. Davis and T. Januszkiewicz, Convex polytopes, Coxeter orbifolds and torus actions, Duke Mathematical Journal 62 (1991), 417-452.

[DS] G. Denham and A.I. Suciu, Moment-angle complexex, monomial ideals, and Massey products, Pure Appl. Math. Q. 3 (2007), 25-60.

[D] N. Dobrinskaya, Loops on polyhedral products and diagonal arrangements, arXiv:0901.2871.

[FT] Y. Félix and D. Tanré, Rational homotopy of the polyhedral product functor, Proc. Amer. Math. Soc. 137 (2009), 891-898.

[F] M. Franz, On the integral cohomology of smooth toric varieties, Proc. Steklov Inst. Math. 252 (2006), $53-62$.

[Ga] T. Ganea, A generalisation of the homology and homotopy suspension, Comment. Math. Helv. 39 (1965), 295-322.

[GL] S. Gitler and S. Lopez de Medrano, Intersections of quadrics, moment-angle manifolds and connected sums, arXiv:0901.2580.

[Go] E.S. Golod, Homologies of some local rings, Dokl. Akad. Nauk SSSR 144 (1962), 479-482.

[GPTW] J. Grbić, T. Panov, S. Theriault and J. Wu, The homotopy types of moment-angle complexes for flag complexes, arXiv:1211.0873., to appear in Trans. Amer. Math. Soc..

[GT1] J. Grbić and S. Theriault, The homotopy type of the complement of the codimension two coordinate subspace arrangement, Russian Math. Surveys 59 (2005), 203-204.

[GT2] J. Grbić and S. Theriault, The homotopy type of the complement of a coordinate subspace arrangement, Topology 46 (2007), 357-396.

[GT3] J. Grbić and S. Theriault, The homotopy type of the polyhedral product for shifted complexes, Adv. Math. 245 (2013), 690-715.

[GW] V. Grujić and V. Welker, Discrete Morse theory for moment-angle complexes of pairs $\left(D^{n}, S^{n-1}\right)$, arXiv:1212.2028

[HRW] J. Herzog, V. Reiner and V. Welker, Componentwise linear ideals and Golod rings, Michigan Math. J. 46 (1999), 211-223.

[IK1] K. Iriye and D. Kishimoto, Decompositions of polyhedral products, Adv. Math. 245 (2013), 716-736. 
[IK2] K. Iriye and D. Kishimoto, Topology of polyhedral products and the Golod property of Stanley-Reisner rings, arXiv:1306.4722.

[J] I.M. James, Reduced product spaces, Ann. of Math. 62 (1955), 170-197.

[Le] J. Lemaire, Algèbres connexes et homologie des espaces de lacets, Lecture Notes in Mathematics, Vol. 422, Springer-Verlag, 1974.

[Li] I. Limonchenko, Combinatorial commutative algebra and topology of moment-angle complexes, $\mathrm{PhD}$ thesis, Moscow State University, 2015.

[LP] Z. Lü and T. Panov, Moment-angle complexes from simplicial posets, Cent. Eur. J. Math. 9 (2011), 715-730.

[M] D. MacGavran, Adjacent connected sums and torus actions, Trans. Amer. Math. Soc. 251 (1979), $235-254$.

[MMP] H. Maeda, M. Masuda and T. Panov, Torus graphs and simplicial posets, Adv. Math. 212 (2007), 458-483.

[MM] J. Milnor and J.C. Moore, On the structure of Hopf algebras, Annals of Math. 81 (1965), 211-264.

[NR] D. Notbohm and N. Ray, On Davis-Januszkiewicz homotopy types. I. Formality and rationalisation, Algebr. Geom. Topol. 5 (2005), 31-51.

[Pa] T. Panov, Moment-angle manifolds and complexes, Trends in Mathematics - New Series. ICMS, KAIST, 12 (2010), 43-69; arXiv:1008.5407.

[PR] T.E. Panov and N. Ray, Categorical aspects of toric topology, Toric topology, 293-322, Contemp. Math. 460, Amer. Math. Soc. 2008.

[Po1] G.J. Porter, Higher order Whitehead products, Topology 3 (1965), 123-135.

[Po2] G.J. Porter, The homotopy groups of wedges of suspensions, Amer. J. Math. 88 (1966), 655-663.

[Q] D.G. Quillen, Rational homotopy theory, Ann. of Math. 90 (1969), 205-295.

[S] R.P. Stanley, $f$-vectors and $h$-vectors of simplicial posets, J. Pure Appl. Algebra. 71 (1991), 319-331.

[T] S. Theriault, Moment-angle manifolds and Panov's problem, Int. Math. Res. Not. (2015), doi:10.1093/imrn/rnu281.

[Tr] A. Trevisan, Generalized Davis-Januszkiewicz spaces, multicomplexes and monomial rings, Homology Homotopy Appl. 13 (2011), 205-221.

School of Mathematics, University of Southampton, Southampton SO17 1BJ, United Kingdom

E-mail address: J.Grbic@soton.ac.uk

School of Mathematics, University of Southampton, Southampton SO17 1BJ, United Kingdom

E-mail address: S.D.Theriault@soton.ac.uk 